\title{
1 IPMK physically binds to the SWI/SNF complex and modulates BRG1 occupancy
}

2

3

4

Jiyoon Beon ${ }^{1, \dagger}$, Sungwook Han ${ }^{1, \dagger}$, Seung Eun Park ${ }^{1}$, Kwangbeom Hyun ${ }^{1}$, Song-Yi Lee ${ }^{2}$, Hyun-Woo Rhee², Jeong Kon $\mathrm{Seo}^{3}$, Jaehoon $\mathrm{Kim}^{1}$, Seyun $\mathrm{Kim}^{1,4, *}$, Daeyoup Lee ${ }^{1, *}$

${ }^{1}$ Department of Biological Sciences, Korea Advanced Institute of Science and Technology (KAIST), Daejeon, 34141, Korea.

2 Department of Chemistry, Seoul National University, Seoul, 08826, Korea.

${ }^{3}$ UNIST Central Research Facilities (UCRF), Ulsan National Institute of Science and Technology (UNIST), Ulsan 44919, Korea.

${ }^{4}$ KAIST Institute for the BioCentury, KAIST, Daejeon 34141, Korea.

*To whom correspondence should be addressed. Tel: +82 42350 2623; Fax: +82 42350 2610; Email: seyunkim@kaist.ac.kr (S.K.), daeyoup@kaist.ac.kr (D.L.).

$\dagger$ These authors contributed equally.

\section{ABSTRACT}

Inositol polyphosphate multikinase (IPMK), a key enzyme in the inositol polyphosphate (IP) metabolism, is a pleiotropic signaling factor involved in major biological events including transcriptional control. In yeasts, IPMK and its IP products were known to promote the activity of SWI/SNF chromatin remodeling complex, which plays a critical role in gene expression by regulating chromatin accessibility. However, the direct linkage between IPMK and chromatin remodelers remains unclear, raising a question on how IPMK contributes to the transcriptional regulation in mammals. By employing unbiased screenings and in vivo/in vitro immunoprecipitations, here we demonstrated that IPMK physically associates with native mammalian SWI/SNF complexes by directly binding to SMARCB1, BRG1, and SMARCC1. Furthermore, we identified the specific domains required for the IPMK-SMARCB1 binding. Notably, using CUT\&RUN and ATAC-seq assays, we discovered that IPMK co-localizes with BRG1 and regulates BRG1 localization as well as BRG1-mediated chromatin accessibility in a genome-wide manner (including promoter-TSS) in mouse embryonic stem cells. Finally, our mRNA-seq analyses revealed that IPMK and SMARCB1 regulate common gene sets, validating a functional link between IPMK and SWI/SNF complex. Together, these findings establish an importance of IPMK in promoter targeting of the SWI/SNF complex, thereby contributing to SWI/SNF-meditated chromatin accessibility and transcription. 


\section{INTRODUCTION}

Inositol polyphosphates are a class of signaling messengers that mediate diverse biological events such as cellular growth, proliferation, and metabolic homeostasis. Inositol polyphosphate multikinase (IPMK) is an essential enzyme for the synthesis of these inositol polyphosphates including inositol tetrakisphosphates ( $\mathrm{IP}_{4}$, both $\operatorname{Ins}(1,3,4,5) \mathrm{P}_{4}$ and $\left.\operatorname{Ins}(1,4,5,6) \mathrm{P}_{4}\right)$ and pentakisphosphates (IP 5 , Ins(1,3,4,5,6)P 5 ) (Chakraborty, Kim, \& Snyder, 2011; Hatch \& York, 2010; Saiardi, Erdjument-Bromage, Snowman, Tempst, \& Snyder, 1999). In addition to its role as a phosphatidylinositol 3-kinase (thereby producing phosphatidylinositol 3,4,5-trisphosphate $\left.\left(\mathrm{PIP}_{3}\right)\right)$, IPMK also non-catalytically controls various signaling factors including the mammalian target of rapamycin (mTOR), AMP-activated protein kinase (AMPK), and TRAF6 (Bang et al., 2012; E. Kim, Ahn, Kim, Lee, \& Kim, 2017; E. Kim, Beon, et al., 2017; S. Kim et al., 2011; Maag et al., 2011; Resnick et al., 2005). These findings suggest that IPMK plays a critical role in coordinating the major biological events.

Increasing evidence strongly indicates that nuclear IPMK act as a key factor in gene expression regulation. IPMK was originally cloned from yeast as Arg82 (yeast IPMK), a gene required for the regulation of arginine metabolism (Bechet, Greenson, \& Wiame, 1970; Dubois, Bercy, \& Messenguy, 1987; Odom, Stahlberg, Wente, \& York, 2000). The physical interaction between Arg82 and the yeast transcription factor MCM1, a yeast homolog of the mammalian serum response factor (SRF), is crucial for the transcriptional control (Bercy, Dubois, \& Messenguy, 1987; Christ \& Tye, 1991; Messenguy \& Dubois, 1993; Odom et al., 2000). In mammals, IPMK-SRF binding was found to be a critical event for SRFdependent gene induction (E. Kim et al., 2013). Furthermore, other functions of nuclear IPMK are mediated by its diverse interactions with p53, steroidogenic factor 1, and CBP/p300 (Blind, 2014; Blind et al., 2014; Malabanan \& Blind, 2016; Xu, Paul, et al., 2013; Xu, Sen, et al., 2013; Xu \& Snyder, 2013).

Chromatin remodeling is essential for the efficient transcription of eukaryotic genes (Kouzarides, 2007; Trotter \& Archer, 2007; Vignali, Hassan, Neely, \& Workman, 2000). Particularly, SWI/SNF is a large family of ATP-dependent chromatin remodeling complexes that have been characterized as transcriptional activators or repressors. These complexes enable the transcription machinery or other transcription factors to gain access to their target genes (Arnaud, Le Loarer, \& Tirode, 2018; Hargreaves \& Crabtree, 2011). In mammalian cells, the canonical SWI/SNF complex contains one of the two mutually exclusive ATPases, BRM (SMARCA2) or BRG1 (SMARCA4), in addition to a core set of subunits consisting of BAF155 (BRG1-associated factor or SMARCC1), SMARCB1 (hSNF5 or INI1), and BAF170 (SMARCC2), as well as four to eight other accessory subunits (Khavari, Peterson, Tamkun, Mendel, \& Crabtree, 1993; W. Wang, Côté, et al., 1996; W. Wang, Xue, et al., 1996). Importantly, this SWI/SNF complex mediates nucleosome structure modifications and regulates the positioning of nucleosomes in an ATP-dependent manner, thereby modulating the accessibility of regulatory proteins. Therefore, the SWI/SNF chromatin remodeling complex is critical for various biological processes, including gene transcription, cell cycle regulation, and cell differentiation (Ho, Jothi, et al., 2009; Ho, Ronan, et al., 2009; Hodges, Kirkland, \& Crabtree, 2016; K. H. Kim \& Roberts, 2014; Tolstorukov et al., 2013; X. Wang, Haswell, \& Roberts, 2014). 
Despite the importance of both inositol polyphosphates and chromatin remodeling in transcriptional regulation, only a few studies have addressed the linkage between inositol polyphosphates and chromatin remodeling. Previous study in yeasts demonstrated that inositol polyphosphates could regulate the nucleosome-sliding activity of chromatin remodeling complexes in vitro (Shen, Xiao, Ranallo, $\mathrm{Wu}, \& \mathrm{Wu}$, 2003). Specifically, inositol tetrakisphosphates and pentakisphosphates $\left(\mathrm{IP}_{4}\right.$ and $\left.\mathrm{IP}_{5}\right)$ stimulate the activity of the SWI/SNF complex, whereas inositol hexakisphosphate ( $\left.\mathrm{IP}_{6}\right)$ inhibits the activity of NURF, ISW2, and INO80 complexes. Another study in yeast illustrated that Arg82 (i.e., a yeast IPMK) mutation which led to defective $\mathrm{IP}_{4}$ and $\mathrm{IP}_{5}$ production, causes inefficient recruitment of the SWI/SNF complex, resulting

80 in impaired chromatin remodeling at the phosphate-responsive PHO5 gene promoter (Steger, Haswell, 81 Miller, Wente, \& O'Shea, 2003). In mammals, inositol hexakisphosphate kinase 1 (IP6K1) was recently 82 found to directly interact with Jumonji domain containing 2C (JMJD2C), a histone demethylase. IP6K1 83 and its product, 5- $\mathrm{IP}_{7}$, appear to mediate $\mathrm{JMJDC}_{2}$-target gene expression in mammalian cells via regulating 84 the chromatin association of JMJDC2 and levels of trimethyl-histone H3 lysine 9 (Burton, Azevedo, 85 Andreassi, Riccio, \& Saiardi, 2013). Taken together, these findings suggest that inositol polyphosphates 86 and their enzymes play an important role in chromatin remodeling and transcription. However, direct 87 linkages between IPMK (which produces inositol polyphosphate) and the chromatin remodeling complex $88 \mathrm{SWI} / \mathrm{SNF}$ have not been previously reported, and it is still unclear whether IPMK contributes the transcriptional regulation of mammals.

To address these issues, we first performed unbiased screening assays and elucidated that the core subunits of the SWI/SNF complex, including SMARCB1 and BRG1, physically interact with IPMK. The physical association between IPMK and SWI/SNF complex was confirmed by our in vitro and in vivo immunoprecipitation assays. Furthermore, the specific binding sites between IPMK and SMARCB1 were mapped in detail. To investigate the biological role of IPMK-SWI/SNF complex binding, we performed various next-generation sequencing. We detected that IPMK and BRG1 were co-localized at the chromatin, especially at the promoter-TSS. Surprisingly, we found that IPMK depletion significantly reduced the global BRG1 occupancy and BRG1-mediated chromatin accessibility, especially at the bivalent promoters. The IPMK depletion also affected the transcription of genes with the reduced BRG1 occupancy and chromatin accessibility at their promoter-TSS. Lastly, we identified that IPMK and SMARCB1 regulate the common set of genes in the same manner. Taken together, our findings demonstrate the direct linkage between IPMK and the SWI/SNF complex (both physical and functional interactions), as well as the crucial role of IPMK in regulating BRG1 occupancy, BRG1-associated chromatin accessibility, and transcription. 


\section{RESULTS}

\section{Identification of IPMK-binding/interacting proteins}

106

107

108

109

110

111

112

113

114

115

116

117

118

119

120

121

122

123

124

125

126

127

128

129

130

To investigate putative IPMK targets, we performed yeast two-hybrid screenings using IPMK as a bait and a human brain cDNA library as prey. The co-transformants of GAL4-DB fusion plasmid pGBKT7IPMK (prey) and GAL4-AD fusion plasmid pACT2-SMARCB1 (bait) resulted in reporter gene activation, thus demonstrating cell growth on selective mediums, whereas co-transformants of pGBKT-7 and pACT2SMARCB1 did not grow (Figure 1A). Approximately 23-36 proteins were identified as potential targets interacting with IPMK (Supplementary Table 1). Among these putative targets, only SMARCB1 was present in both duplicates of the yeast two-hybrid screening (Supplementary Table 1). These results indicate SMARCB1, a core subunit of SWI/SNF chromatin remodeler, as a potential candidate for IPMK-binding proteins.

To further identify potential target proteins associating with IPMK in mammalian cells, we performed an in vivo proximity-labeling approach using an engineered variant of ascorbate peroxidase (APEX2) fused to IPMK (APEX2-mediated proximity labeling). The IPMK-APEX2 or APEX2 neighboring proteins were biotinylated, enriched with streptavidin beads, and analyzed via mass spectrometry (Figure 1B). A total of 455 IPMK-associated candidate proteins was identified by comparing neighboring proteins of APEX2IPMK with those of APEX2 only (background, used as a negative control) using two-fold enrichment score differences. Interestingly, by performing ConsensusPathDB (Herwig, Hardt, Lienhard, \& Kamburov, 2016) using the IPMK-associated candidates, we detected the enriched protein complex-based sets related to BRG1-, BAF-, or SWI/SNF complex-associated complexes (Supplementary Table 2). Notably, among these candidates, we detected SWI/SNF complex-associated factors, including SMARCB1 (BAF47), BRG1 (SMARCA4), SMARCC2 (BAF170), ARID1A (BAF250A), PBRM1 (BAF180), and SMARCC1 (BAF155), as IPMK-proximal/binding target proteins (Figure 1C). Furthermore, among these SWI/SNF complex subunits, we found that SMARCB1 most significantly interacts with or being proximal to IPMK (Figure 1C), consistent with the yeast two-hybrid screening results (Figure 1A). Intriguingly, we also detected core histones (histone H2B, H3.1, and H4) as IPMK-proximal/binding targets (Figure 1-figure supplement 1), supporting that IPMK binds/proximal with SWI/SNF complex, which binds to nucleosomes in vivo. Collectively, these results from two unbiased screening experiments (including yeast two-hybrid screening assay and APEX2-mediated proximity-labeling-based proteomics) strongly indicate that IPMK physically associates with the SWI/SNF complex.

\section{Physical interaction between IPMK and core subunits of SWI/SNF complex}

To confirm the physical interaction between IPMK and SMARCB1, we first performed an in vitro binding assay using recombinant IPMK and SMARCB1 proteins. Notably, we detected a direct proteinprotein interaction between IPMK and SMARCB1 in vitro (Figure 2A, see lane 2 and lane 4). To confirm the physical association between IPMK and core subunits of SWI/SNF (BAF) complex, we performed 
140 binary protein interactions assays with baculovirus-mediated expression. We co-infected Sf 9 insect cells

141 with baculoviruses expressing FLAG-IPMK and untagged individual subunits of SWI/SNF complex,

142 including SMARCB1, BRG1, BAF155 (SMARCC1), and BAF170 (SMARCC2). Then, we performed

143 FLAG M2 agarose immunoprecipitation and immunoblotting. Importantly, we detected direct protein-

144 protein interactions between IPMK-SMARCB1, IPMK-BRG1, and IPMK-BAF155 in vitro, while we did

145 not detect IPMK-BAF170 interactions (Figure 2B, see lane 7 and lane 8). Together, these results indicate

146 that IPMK directly/individually binds to SMARCB1, BRG1, and BAF155 in vitro.

147 To investigate whether these IPMK-SMARCB1/BRG1/BAF155 interactions also occurred in vivo, we

148 performed co-immunoprecipitation experiments using mammalian cells. Importantly, we detected a direct

149 association of endogenous IPMK and SMARCB1 in mouse embryonic stem cells (mESCs) (Figure 2C and

150 D) and in mouse embryonic fibroblasts (MEFs) (Figure 2-figure supplement 1A-C). Consistent with our

151 in vitro binding assays (Figure 2A and B), we detected SMARCB1, BRG1, and BAF155 in endogenous

152 IPMK immunoprecipitates (Figure 2C) and FLAG-IPMK overexpressed immunoprecipitates (Figure 2E)

153 in mESCs. Intriguingly, we detected SMARCB1, BRG1, and BAF170 in endogenous IPMK

154 immunoprecipitates in MEFs (Figure 2-figure supplement $1 \mathrm{~A}$ and $\mathrm{B}$ ). To determine the specificity of the

155 IPMK-SMARCB1 physical interaction, RNAi-mediated knockdown of Ipmk (IpmkKD) and Smarcb1

156 (Smarcb/KD) was conducted in mESCs and MEFs. We first confirmed the successful knockdown of both

157 Ipmk and Smarcb1 by quantifying the protein levels (Figure 2F, Figure 2-figure supplement 1D and E).

158 In addition, we observed that Ipmk knockdown did not affect the protein levels of SWI/SNF complex

159 subunits, and Smarcbl knockdown did not affect the protein levels of IPMK both in mESCs and MEFs

160 (Figure 2-figure supplement 1D). Importantly, in IPMK immunoprecipitates, a significant reduction in

161 SMARCB1 signals was observed in IpmkKD mESCs compared to the control (EgfpKD) mESCs (Figure

162 2F). We also found that SMARCB1 and BRG1 signals in IPMK immunoprecipitates were significantly

163 decreased upon SmarcbKD MEFs compared to the control (EgfpKD) MEFs (Figure 2-figure supplement

164 1E). Lastly, in SMARCB1 immunoprecipitates, a significant reduction of IPMK signals was detected in

165 IPMK-null MEFs (Figure 2-figure supplement 1C). Together, these results indicate that IPMK directly

166 associates with the core subunits of SWI/SNF complex in vivo (IPMK-SMARCB1/BRG1/BAF155 in

167 mESCs; IPMK-SMARCB1/BRG1/BAF170 in MEFs; IPMK-SMARCB1 binding is specific and observed

168 both in mESCs and MEFs).

169 Considering our previous results (unbiased screenings, in vitro, and in vivo immunoprecipitation assays),

170 it is highly plausible that IPMK physically associates with SWI/SNF complex. To confirm this, we first

171 performed a co-immunoprecipitation assay by overexpressing IPMK and SMARCB1 in human embryonic

172 kidney (HEK)-293T cells. Consistently, we observed a physical interaction between IPMK and SMARCB1

173 (Figure 2-figure supplement 1F). Next, we performed GST (-IPMK) pull-down assays by overexpressing

174 GST-IPMK or GST alone in HEK293T cells. Notably, compared to GST alone, we detected core subunits

175 of SWI/SNF (BAF)/PBAF complexes, including SMARCB1, BAF155, BAF170, PBRM1, BAF250A, and

176 BRM, in GST-IPMK pulled-down samples (Figure 2-figure supplement 1G), as consistent with our

177 APEX2-mediated proximity labeling assays (Figure 1C). Lastly, we purified native SWI/SNF (BAF) 
complexes from FLAG-DPF2 cell lines (Figure 2 - figure supplement 1H), co-incubated the purified native SWI/SNF complexes, and purified GST-IPMK or GST alone, then performed GST pull-down assays. As expected, we detected the core subunits of SWI/SNF complexes (including SMARCB1, BRG1, BAF155, and BAF170) in GST-IPMK pulled-down samples (Figure 2-figure supplement 1I). Collectively, our combined results (unbiased screenings, in vitro/in vivo immunoprecipitation, and in vitro pull-down assays) strongly imply that IPMK physically associates with mammalian SWI/SNF complexes by directly binds to SMARCB1, BRG1, and BAF155 (SMARCC1).

\section{Mapping the reciprocal binding sites of IPMK and SMARCB1}

Among three IPMK-binding proteins (SMARCB1, BRG1, and BAF155), SMARCB1 exhibited the most robust interaction with IPMK (Figure 1 and 2). Regarding this, we conducted yeast two-hybrid assays to identify the specific binding domains of SMARCB1 required for interacting with IPMK. Various prey vectors encoding different SMARCB1 domains were cloned, and two-hybrid analyses were performed. Interestingly, the prey vectors expressing amino acids of 99-245, 99-319, and full-length SMARCB1 showed positive signals in the two-hybrid system (Figure 3-figure supplement 1A), indicating that the Rpt1 and Rpt2 domains of SMARCB1 participate in the protein-protein interactions of SMARCB1 and IPMK

To further dissect the reciprocal binding sites required for SMARCB1-IPMK physical binding, various SMARCB1 constructs were designed and overexpressed in HEK293T cells. Based on the domain map of SMARCB1 (Figure 3A, top), we first designed SMARCB1 constructs with C-terminal deletion (Figure 3A, middle). We confirmed that the Rpt1 domain of SMARCB1 is essential for IPMK interaction by immunoprecipitating the overexpressed deletion constructs (Figure 3B, compare lane 3 to lane 2, 4, and 5). We then generated additional N-terminal deleted constructs of SMARCB1 (Figure 3A, bottom). Consistent with the results of yeast two-hybrid assays (Figure 3-figure supplement 1A), we observed that the constructs containing the Rpt1 or Rpt2 domains associate with IPMK (Figure 3C, lanes 2, 3, 4, and 5 show positive immunoblotting signals). By independently overexpressing each SMARCB1 domain, we found that both the Rpt1 and Rpt2 domains of SMARCB1 could bind to IPMK (Figure 3D and E, see lane 3, 4, and 6). Additionally, we dissected Rpt1 and Rpt2 domains into $\beta$ sheets and $\alpha$ helices based on their structures. In Rpt1, two $\beta$ sheets and two helices are required for IPMK-binding (Figure 3-figure supplement 1B, compare lane 4 to lane 2 and 3). In Rpt2, only two $\beta$ sheets but not $\alpha$ helices were bound to IPMK (Figure 3-figure supplement 1C, compare lane 2 and lane 3). These observations (for IPMKbinding, both sheets and helices of Rpt1 or/and only sheets of Rpt2 are required) were supported by overexpressing combinations of Rpt1 and Rpt2 domains (Figure 3-figure supplement 1D, lanes 3 and 4 show positive signals). Lastly, this was further confirmed by the fact that SMARCB1 lacking Rpt1 and 
Reciprocally, to identify which IPMK domains are required for SMARCB1 binding, we designed several GST-tagged constructs of IPMK (Figure 3G) and conducted immunoprecipitation experiments. Intriguingly, we found that IPMK-SMARCB1 binding was primarily mediated by three IPMK regions, including exon 3, exon 4, and exon 6 (Figure 3F, lanes 4, 5, 7, and 8 show positive signals, and 3G), which comprises the inositol binding site and the kinase domain. Taken together, our results elucidated the specific reciprocal biding sites of IPMK-SMARCB1 interaction.

\section{Co-localization of IPMK and BRG1}

Our results demonstrated that IPMK directly binds to the core subunits of mammalian SWI/SNF complexes (SMARCB1, BRG1, and BAF155) and physically associates with native SWI/SNF complexes.

225 Regarding this, one can speculate that IPMK may play an important role in chromatin regulation. However, 226 the region where this IPMK-SWI/SNF interaction occurs in vivo and the detailed localization and the role 227 of IPMK in the chromatin remains elusive. To decipher these issues, we first conducted a chromatin 228 fractionation assay using mESCs and MEFs. Notably, we found that IPMK is evenly distributed in all three fractions (cytoplasm, nucleoplasm, and chromatin), whereas SMARCB1 and BRG1 primarily reside in the chromatin fraction both in mESCs and MEFs (Figure 4A and Figure 4-figure supplement 1A-C). To further investigate whether IPMK and SMARCB1 expressions affect each other's distribution, RNAimediated knockdown of Ipmk (IpmkKD) and Smarcb1 (Smarcb1KD) was conducted before the chromatin

233 fractionation assay. We observed that the distribution of SMARCB1 was unaffected by IpmkKD (Figure

234 4A), and the distribution of IPMK was unaffected by Smarcb1KD (Figure 4-figure supplement 1C). 235 Together, these results indicate that IPMK, BRG1, and SMARCB1 reside together in the chromatin.

236 We then sought to determine where this event (IPMK, BRG1, and SMARCB1 localizing at the chromatin) takes place within the chromatin. To investigate the localization of BRG1 and IPMK within the chromatin, we performed CUT\&RUN (cleavage under targets and release using nuclease) assays (Skene \& Henikoff, 2017) in mESCs. In accordance with our results of the IPMK-SWI/SNF complex's physical association, we found that IPMK was co-localized with BRG1 in a genome-wide manner (Figure 4B and

241 C). Next, we performed peak annotation to analyze the genomic regions (e.g., promoters or intergenic regions) enriched with CUT\&RUN peaks. Given that BRG1, a catalytic subunit of the SWI/SNF complex,

243 is known to localize at the promoter-transcription start site (TSS) (de Dieuleveult et al., 2016), we 244 confirmed that BRG1 was significantly enriched at promoters of the mouse genome (Figure 4D and E). 245 Notably, we detected that IPMK was also significantly enriched at promoters (Figure 4D and E). 246 Collectively, these results strongly indicate that IPMK and BRG1 are co-localized at the chromatin, 247 particularly at the promoter region, which further support our previous results of physical association 248 between IPMK and SWI/SNF complex.

250 IPMK regulates the BRG1 occupancy and impacts the BRG1-mediated chromatin accessibility

251 To further elucidate the role of IPMK in BRG1 localization, we performed BRG1 CUT\&RUN assays 
upon IpmkKD and compared them to EgfpKD (control) in mESCs. Interestingly, we observed a decreased

253 BRG1 occupancy upon IpmkKD at specific promoter-TSS regions with enriched IPMK (Figure 4B and F).

254 Strikingly, we found that the genome-wide BRG1 localization was severely disrupted (decreased BRG1

255 occupancy) upon IpmkKD at BRG1 CUT\&RUN peaks with low BRG1 enrichment in EgfpKD mESCs

256 (bottom half of the heatmaps, termed as Low) (Figure 4C, G, and H). In addition, we detected that BRG1

257 CUT\&RUN peaks' genomic distributions were unaffected by IpmkKD (Figure 4D and E), suggesting that

258 IpmkKD do not affect the global distribution (changes in peak positions) of BRG1 but impacts the global 259 occupancy of BRG1.

260 It is previously known that BRG1 regulates chromatin accessibility at NFR (nucleosome free regions)

261 of TSS in mESCs (de Dieuleveult et al., 2016). To investigate the effect of IpmkKD-induced decreased

262 BRG1 occupancy on chromatin accessibility, we performed ATAC-seq (assay for transposase-accessible

263 chromatin using sequencing) upon IpmkKD and compared them to EgfpKD (control) in mESCs. Notably,

264 we observed that both ATAC-seq signals and BRG1 occupancy were reduced upon IpmkKD at promoter-

265 TSS regions of Nanog (Figure 4F). Furthermore, we observed some discrepancies in major peak positions

266 when comparing BRG1 CUT\&RUN peaks ( $E g f p \mathrm{KD}$, termed as BRG1 peaks) and ATAC-seq peaks

267 (EgfpKD, termed as ATAC peaks) (Figure 4F). To precisely assess the effect of IpmkKD-induced decreased

268 BRG1 occupancy on chromatin accessibility, we assigned ATAC peaks to the nearby (within 2kb) BRG1

269 peaks and selected these BRG1 peaks for further analysis (we excluded BRG1 peaks without nearby ATAC

270 peaks) (Figure G). In addition, BRG1 peaks containing or assigned with multiple ATAC peaks were

271 included without deduplication to match the same ordering as ATAC peaks (the same ordering - alignment

272 of heatmaps' row - was applied for BRG1 and ATAC peaks in Figure 4G). As expected, we observed that

273 the global BRG1 occupancy was reduced upon IpmkKD at BRG1 peaks with low BRG1 intensity (bottom

274 half of the heatmaps, termed as Low) (Figure G and H), consistent with our previous observations (Figure

275 4C). Surprisingly, at Low BRG1 peaks (Low), we observed that both BRG1 occupancy and BRG1-

276 mediated chromatin accessibility (ATAC-seq signals closest to the BRG1 CUT\&RUN peaks) were

277 significantly reduced upon IpmkKD in a genome-wide manner (Figure 4G and H). Furthermore, the reduced

278 BRG1 and BRG1-associated chromatin accessibility (ATAC-seq) upon IpmkKD were also detected at

279 promoter-TSS regions (Figure 4I), consistent with the previous study (BRG1 primarily maintains chromatin

280 accessibility at promoter-TSS regions) (de Dieuleveult et al., 2016). Taken together, these results indicate

281 that IPMK regulates the global BRG1 occupancy and corresponding BRG1-mediated chromatin

282 accessibility in mESCs.

284 IPMK plays an important role in BRG1 localization and chromatin accessibility at promoter-TSS

285 We next focused on the promoter-TSS regions, where BRG1 and IPMK were significantly enriched

286 (Figure 4D and E). To further dissect the genome-widely decreased BRG1 occupancy upon IpmkKD, we

287 classified the distinct clusters of promoter-TSS based on the promoter types and changes in BRG1 intensity

288 at defined regions relative to TSS positions (Figure 5C-G). Initially, by analyzing MNase-seq (chromatin

289 digestion with micrococcal nuclease combined with sequencing) and ATAC-seq data in mESCs (EgfpKD), 
we observed nucleosome-depleted/chromatin accessible regions, known as NFR (nucleosome free regions), and detected -1 and +1 nucleosomes near TSS (Figure 5A, left). We also confirmed that BRG1 was abundant at TSS by analyzing BRG1 CUT\&RUN assays (Figure 5A, left). Based on the signal intensity of ChIP-seq (chromatin immunoprecipitation sequencing) against histone H3K4me3 and H3K27me3, we classified promoters into three types: H3K4me3-Low, H3K4me3-Only (high H3K4me3, low H3K27me3), and Bivalent (high H3K4me3, high H3K27me3). Considering nucleosome intensity and ATAC-seq signals, we detected that chromatin was highly accessible in H3K4me3-Only, moderately accessible in bivalent, and inaccessible in H3K4me3-Low promoters (Figure 5A, right). Next, we categorized the 9,042 TSS with decreased BRG1 occupancy upon IpmkKD (1.5 fold changes in BRG1 occupancy compared to EgfpKD, see also Figure 5D, left Total) into the three promoter types (Figure 5B). Since our goal was to examine the IpmkKD-induced 'decreased' BRG1 occupancy (changes from enriched to depleted BRG1 signal upon IpmkKD as seen in Figure 4C and G-I), we excluded the H3K4me3-Low promoters, which exhibited the extremely low BRG1 signals (Figure 5A, top right). Furthermore, regarding the fact that TSS divided the BRG1 intensity into two (Figure 5A, top left), we defined two genomic regions (Upstream and Downstream) that coincided with the two major BRG1 intensity (which also coincided with -1 and +1 nucleosomes), respectively (Figure 5A, top). We then categorized the 9,042 TSS with IpmkKD-induced decreased BRG1 occupancy into five clusters based on the combinatorial changes in BRG1 levels upon IpmkKD at the previously defined two genomic regions (Figure 5C, left and middle) and subdivided these five clusters based on two promoter types (Figure 5C, right). Regarding the number of promoter-TSS with decreased BRG1 level upon IpmkKD, H3K4me3-Only promoters (Figure 5B and C, right) and Cluster2/3 (Figure 5C) occupied a large proportion. In accordance with our defined classification (Figure 5C, left), we detected that BRG1 occupancy was reduced upon IpmkKD at both promoters and five distinctive clusters (Figure $5 \mathrm{D})$.

Previously, it is reported that BRG1, localized at -1 nucleosome in wide NFR (median length 808 bp) of H3K4me3-Only and bivalent promoters, positively regulates the chromatin accessibility of NFR, whereas BRG1 that localized at +1 nucleosome in narrow NFR (median length 28 bp) of H3K4me3-Only promoters tends to inhibit the chromatin accessibility of NFR in mESCs (de Dieuleveult et al., 2016). To elucidate the effect of IpmkKD-induced decreased BRG1 occupancy on chromatin accessibility at two promoters and five clusters, we analyzed two ATAC-seq data of ours (IpmkKD) and publically released (Brg1KD, GSE64825). We calculated the differential ATAC-seq signals (KD vs. controls) at Center regions, where ATAC-seq intensity is highly enriched (Figure 5A). Consistent with our previous genome-wide results (Figure 4G-I), we found that ATAC-seq signals (chromatin accessibility) were significantly reduced upon IpmkKD at two promoters (Figure 5E) and most clusters (Figure 5F), where these promoters/clusters were defined by decreased BRG1 occupancy upon IpmkKD. Notably, we observed that ATAC-seq signals (chromatin accessibility) were decreased similarly upon IpmkKD and $\operatorname{Brg} 1 \mathrm{KD}$ at two promoters (Figure 5E) and five clusters (Figure 5F). Since the reduced BRG1 occupancy upon IpmkKD partially mimics the $B r g 1 \mathrm{KD}$, the similar result upon IpmkKD and BrglKD further supports that IPMK plays a vital role in 
the ATAC-seq signals at bivalent promoters were more reduced upon IpmkKD compared to those at H3K4me3-Only promoters (Figure 5E and F). Furthermore, we detected that the ATAC-seq signals at Cluster2 of bivalent promoters were more reduced upon IpmkKD than other clusters (Figure $5 \mathrm{~F}$ ), consistent with the fact that in bivalent promoters, BRG1 is localized at the -1 nucleosome and maintains the chromatin accessibility (de Dieuleveult et al., 2016). Although H3K4me3-Only promoters also contain Cluster2, we did not detect the robust decrease in ATAC-seq signals at Cluster2 of H3K4me3-Only promoters, unlike the bivalent promoter case (Figure 5F). This discrepancy may be due to the different BRG1 occupancy in Upstream (-1 nucleosome) and Downstream (+1 nucleosome) regions of Cluster2 in EgfpKD mESCs; BRG1 is highly enriched at Upstream compare to Downstream regions in bivalent promoters, while in H3K4me3-Only promoters, BRG1 levels are relatively similar at both Up/Downstream regions (Figure 5D). Interestingly, although we applied the same criteria when categorizing the five clusters, H3K4me3-Only and bivalent promoters exhibited different BRG1 localizations in EgfpKD mESCs at Cluster2 and Cluster3, indicating that these two promoters each possess distinct BRG1 localizations in mESCs. Together, these results suggest that IPMK plays a pivotal role in maintaining the chromatin accessibility of bivalent promoters, particularly by safeguarding the BRG1 occupancy at the -1 nucleosome.

By comparing with the BRG1 unchanged locus (Figure 5G, left), we confirmed the close association between reduced BRG1 level upon IpmkKD and decreased ATAC-seq signals upon IpmkKD and Brgl $1 \mathrm{KD}$ at specific loci of $\mathrm{H} 3 \mathrm{~K} 4 \mathrm{me} 3$-Only and bivalent promoters (Figure 5G and H). However, at a specific locus, we noticed that some BRG1 peaks do not coincide with Up/Downstream regions (Figure 5H), indicating that our promoter-TSS classification using Up/Downstream regions with fixed length does not include promoter-TSS with far away BRG1 peaks. To overcome this, we first selected BRG1 CUT\&RUN peaks $(E g f p \mathrm{KD})$ that reside in close proximity to TSS $( \pm 1 \mathrm{~kb})$ and then newly classified the promoter-TSS into six clusters depending on the position of BRG1 peaks relative to the position of TSS (Figure 5-figure supplement 1A and B). Among six clusters, Cluster1 and 5, which contain BRG1 peaks at TSS and upstream of TSS, respectively, occupied a large proportion (Figure 5-figure supplement 1B). Interestingly, we observed that BRG1 localizations were remarkably similar at Cluster1/2/3, and independently at Cluster $4 / 5$

354 (Figure 5-figure supplement 1A). To simplify the clustering, we merged Cluster1/2/3 as ClusterC and Cluster4/5 as ClusterL (Cluster6 is equivalent to ClusterR) (Figure 5-figure supplement 1C and D).

356 Consistently, ClusterC and L, which contain BRG1 peaks at their TSS and upstream of their TSS, respectively, were predominant (Figure 5-figure supplement 1D). We then divided each cluster depending on their decreased or increased BRG1 level by calculating the differential BRG1 level (IpmkKD vs. EgfpKD) at the BRG1 peaks corresponding to TSS (Figure 5-figure supplement 1E), instead of at the defined $\mathrm{Up} /$ Downstream regions (Figure 5B and C). Consistent with the genome-wide decrease in BRG1 occupancy at the promoter-TSS (Figure 4I), we found that $60-70 \%$ of clusters exhibited decreased BRG1 level upon IpmkKD (Figure 5-figure supplement 1E). Furthermore, most of these IpmkKD-induced decreased BRG1 clusters were identified as H3K4me3-Only promoters (Figure 5-figure supplement 1F). 
first confirmed a significant reduction in BRG1 occupancy upon IpmkKD at the pre-defined BRG1decreased clusters/promoters (Figure 5-figure supplement 1G). Next, we calculated differential ATACseq signals (IpmkKD vs. $E g f p \mathrm{KD}$ and $B r g l \mathrm{KD}$ vs. control) at ATAC-seq peaks ( $E g f p \mathrm{KD})$ that reside near the TSS $( \pm 0.5 \mathrm{~kb})$ of each BRG1-decreased cluster/promoter. Consistent with our previous results (Figure $5 \mathrm{E}$ and F), we observed that both IpmkKD and BrglKD induced similarly decreased ATAC-seq levels at both promoters and all three clusters (Figure 5-figure supplement 1H). When comparing H3K4me3-Only and bivalent promoters, we observed that ClusterC exhibited a similar reduction, while ClusterL/R exhibited more reduction at bivalent promoters upon IpmkKD (Figure 5-figure supplement 1H, blue). Similarly, we observed that all three clusters exhibited more reduction at bivalent promoters compared to H3K4me3-Only promoters upon Brg1KD (Figure 5-figure supplement 1H, green). Lastly, among bivalent promoters, we detected that ClusterL (very similar to Cluster2 in Figure 5) exhibited the most significant reduction in ATAC-seq signals upon IpmkKD (Figure 5-figure supplement 1H).

Collectively, our two alternative analyses suggest that IPMK depletion cause the most severe impacts on the chromatin accessibility at bivalent promoters, which are strongly associated with the IpmkKDinduced decreased BRG1 occupancy at -1 nucleosomes. Taken together, these findings indicate that IPMK regulates BRG1 occupancy and BRG1-mediated chromatin accessibility at promoter-TSS regions.

\section{Loss of IPMK partially affects transcription via disrupted BRG1 localization and chromatin} accessibility at promoter-TSS

To investigate the effect of IpmkKD-driven disrupted BRG1 occupancy and chromatin accessibility on the transcription, we performed high-throughput mRNA sequencing (mRNA-seq) using mESCs with RNAi-mediated knockdown of Ipmk (IpmkKD). We next calculated the differential mRNA expression levels (IpmkKD vs. EgfpKD) of genes having the promoters with decreased BRG1/ATAC-seq intensity upon IpmkKD (Figure 5D and E, Total). The mRNA expression of genes with both promoter types was down-regulated upon IpmkKD (Figure 5-figure supplement 1I). Intriguingly, we detected that mRNA expression of genes with bivalent promoters was significantly down-regulated upon IpmkKD, compared to H3K4me3-Only promoters (Figure 5-figure supplement 1I). This discrepancy may arise from our previous results that IpmkKD impacted the bivalent promoter-TSS's chromatin accessibility more than those of H3K4me3-Only promoters (Figure 5E, F, and Figure 5-figure supplement 1H). To check the decreased BRG1/ATAC-seq-associated down-regulation of gene expressions upon IpmkKD, we performed a real-time quantitative polymerase chain reaction (RT-qPCR), a conventional method to check the gene expression. We first determined the successful knockdown of Ipmk (Figure 6A). Next, we confirmed that mRNA expression of genes, exhibiting decreased BRG1 occupancy upon IpmkKD and reduced ATAC-seq signals upon IpmkKD/BrglKD at H3K4me3-Only (Nmrall) or bivalent (Phactr3) promoters, were significantly down-regulated upon IpmkKD (Figure 6B). To examine this in a genome-wide manner, we identified differentially expressed genes (DEGs) by comparing the gene expression of IpmkKD with that of control (EgfpKD) cells and identified 300 down-regulated DEGs (Figure 6C). Notably, we observed that 
reduced near TSS of down-regulated DEGs upon IpmkKD compared to EgfpKD (Figure 6D). We further confirmed this by monitoring the specific down-regulated DEG loci, including Phactr3, Lrrc61, and Arhgap44 (Figure 6B, right, E, and F). Together, these results suggest that IPMK maintains the expression of a subset of genes by safeguarding the appropriate BRG1 occupancy and BRG1-mediated chromatin accessibility at promoter-TSS in mESCs.

408

\section{IPMK and SMARCB1 regulate a common set of genes in mESCs and MEFs}

410

To investigate the functional interactions between IPMK and SMARCB1 in a transcription aspect, we performed mRNA-seq using mESCs upon IpmkKD or Smarcb1KD. Using the same criteria (fold change $\geq$ 1.5 and $P$-value $\leq 0.05$ ), we defined differentially expressed genes (DEGs) by comparing the gene expression of IpmkKD or Smarcb1KD cells with that of control (EgfpKD) cells. A total of 217 up-regulated DEGs and 300 down-regulated DEGs were identified in IpmkKD cells (Figure 6C), and 248 up-regulated DEGs and 291 down-regulated DEGs were identified in Smarcb1KD cells (Figure 6G). Given that our previous results demonstrated the physical binding between IPMK and SMARCB1, we hypothesized that IPMK and SMARCB1 would functionally interact with each other. To check this hypothesis, we classified DEGs of IpmkKD cells into six clusters based on the combinatorial mRNA expression changes upon IpmkKD and Smarcb1KD (Figure 6H and Supplementary Table 3). Among these six clusters, Cluster1/2/3 represent the up-regulated DEGs upon IpmkKD, whereas Cluster4/5/6 represent the down-regulated DEGs upon IpmkKD. Specifically, Cluster1 and Cluster5 each represent the up-regulated DEGs and downregulated DEGs shared by both IpmkKD and Smarcb1KD cells, respectively. In contrast, Cluster2 and Cluster4 each represent the oppositely regulated DEGs upon IpmkKD and Smarcb1KD; Cluster2 contains up-regulated DEG upon IpmkKD and down-regulated DEG upon SmarcblKD, while Cluster4 contains down-regulated DEGs upon IpmkKD and up-regulated DEG upon SmarcblKD. The remaining DEGs of IpmkKD cells were marked as Cluster3/6. Surprisingly, DEGs that were regulated in the same manner (both up-regulated Cluster1 and both down-regulated Cluster5, 18.8\%) were far more abundant than DEGs that were oppositely regulated (Cluster2 and Cluster4, 0.6\%) (Figure 6H and I). If we assume that IPMK is not functionally related to SMARCB1, the number of DEGs regulated in the same fashion or the opposite fashion should be similar. However, we observed that DEGs regulated in the same manner are much more abundant ( $\sim 32$ fold more) than DEGs that are oppositely regulated upon Ipmk/SmarcblKD, indicating that IPMK and SMARCB1 regulate the common gene sets in mESCs. In addition, we observed that both upregulated and down-regulated DEGs of IpmkKD cells significantly overlapped with those of Smarcb1KD cells (Figure 6J), further supporting the functional linkage between IPMK and SMARCB1. Finally, we performed gene ontology (GO) analysis using genes representing Cluster1 and Cluster5. Despite the similar number of genes in Cluster1 $(n=50)$ and Cluster5 $(n=47)$, the GO terms of Cluster5 were significantly enriched compared to those of Cluster1 (Figure 6-figure supplement 1A), suggesting that down-regulated DEGs regulated by IPMK/SMARCB1 are more closely associated/related to each other compared to up- 
440 sets in the same manner, and among these genes, down-regulated DEGs are closely associated with each 441 other.

442 To further investigate whether the functional interaction between IPMK and SMARCB1 is a specific 443 feature of mESCs or a more general feature, we performed identical analyses in MEFs (NIH3T3). We first 444 defined DEGs; a total of 209 up-regulated DEGs and 275 down-regulated DEGs were identified upon $445 I p m k \mathrm{KD}$, and 404 up-regulated DEGs and 678 down-regulated DEGs were identified upon SmarcblKD 446 (Figure 6-figure supplement 1B). We then defined the six clusters with the same procedure described for 447 the mESCs. Consistent with the results obtained in the mESCs (Figure 6H and I), DEGs regulated in the 448 same manner (both up-regulated Cluster1 and both down-regulated Cluster5, 23.8\%) were much more 449 abundant than DEGs that are oppositely regulated (Cluster 2/4, 1.7\%) (Figure 6-figure supplement 1C, D, 450 and Supplementary Table 4). Furthermore, we confirmed that both up-regulated and down-regulated DEGs 451 of IpmkKD cells significantly overlapped with those of Smarcb1KD cells (Figure 6-figure supplement 452 1E), consistent with the results obtained from mESCs (Figure 6J). Taken together, the fact that IPMK and 453 SMARCB1 regulate a common set of genes in the same fashion strongly implies the transcription-related 454 functional interaction between IPMK and SMARCB1 both in mESCs and MEFs. 


\section{DISCUSSION}

Using two unbiased screening (yeast two-hybrid and APEX2 proximity labeling), we detected SMARCB1 and other core subunits of the mammalian SWI/SNF complex (BRG1, BAF155, BAF170, ARID1A, and PBRM1) as IPMK-proximal/binding targets. Notably, our binary protein interaction assays showed that IPMK directly binds to SMARCB1, BRG1, and BAF155, individually. Furthermore, in vivo and in vitro immunoprecipitation assays confirmed that IPMK physically associates with the native SWI/SNF complexes. Detailed mapping studies further revealed reciprocal interactions between the Rpt domains of SMARCB1 and the IP kinase domain of IPMK. In accordance with our previous finding (physical association of IPMK-SWI/SNF complex), our CUT\&RUN analysis showed that IPMK is colocalized with BRG1 globally, especially at the promoter-TSS. Surprisingly, by performing CUT\&RUN and ATAC-seq, we discovered that the depletion of IPMK severely perturbed (decreased) the genome-wide BRG1 localizations and corresponding BRG1-mediated chromatin accessibility (ATAC-seq signals). By categorizing promoter-TSS, we found that IPMK depletion significantly affected the chromatin accessibility at the bivalent promoters, which were associated with the disrupted BRG1 occupancy at the 1 nucleosome. Using RT-qPCR and mRNA-seq, we detected that the IPMK loss also affects the transcription of genes exhibiting disrupted BRG1/ATAC-seq levels at their promoter-TSS. Lastly, our mRNA-seq analyses also demonstrated that IPMK and SMARCB1 regulate common gene sets, implying a functional link between IPMK and SWI/SNF complex. Taken together, these results elucidate the critical role of IPMK in regulating BRG1 localizations and BRG1-mediated chromatin accessibility through the physical association between IPMK and SWI/SNF complex (Figure 7).

The physical association between IPMK and SWI/SNF complex is very engaging regarding the structural aspect. According to the recently published cryo-EM-based structure of nucleosome-bound human SWI/SNF complexes (He et al., 2020), the nucleosome is sandwiched by the ATPase module of BRG1 and the base module of SMARCB1 (C-terminal $\alpha$ helix of the Rpt2 domain). BRG1 grasps the upper side of a nucleosome, whereas SMARCB1 binds to the bottom surface of a nucleosome through their positively charged four arginine residues at the C-terminal $\alpha$ helix of Rpt 2 domain interacting with the acidic patch of histone octamers (He et al., 2020) (Figure 7-figure supplement 1). Considering this, we revisited our domain mapping results, showing that Rpt1 (all regions including N-terminal $\beta$ sheets and Cterminal $\alpha$ helices) and Rpt2 (N-terminal $\beta$ sheets) domains of SMARCB1 and reciprocally, the Exon3/4/6 of IPMK (consists of $\sim 71 \%$ of IPMK proteins) are required for IPMK-SMARCB1 binding (Figure 7A, left). The N-terminal $\beta$ sheets of the Rpt2 domain seem to be quite buried at the frontal view of nucleosomebound SWI/SNF complex (He et al., 2020). However, if we rotate the structure and see the back view, we could observe that the beginning part (that is close to $\mathrm{C}$-terminal $\alpha$ helices of Rpt 1 ) of $\mathrm{N}$-terminal $\beta$ sheets of Rpt2 is being exposed, which may allow some space for IPMK binding. More importantly, we also noticed some space near the Rpt1 domain of SMARCB1 and nucleosomal DNA exit sites, which may provide space for IPMK binding. Intriguingly, this space is positioned near BRG1 and BAF155, which can 

(BAF170), ARID1A (BAF250A), PBRM1 (BAF180), BAF155 (SMARCC1), and core histones (histone H2B, H3.1, and H4) as IPMK-proximal/binding target proteins (Figure 1B, C, and Figure 1-figure supplement 1). Together, we believe that IPMK would be positioned at specific sites within SWI/SNF complex, where it meets the three conditions: (1) sites where IPMK directly binds to the Rpt1 domain (all regions containing N-terminal $\beta$ sheets and C-terminal $\alpha$ helices) of SMARCB1, (2) sites where IPMK directly binds to SMARCB1, BRG1, and BAF155, and (3) sites proximal to the various subunits of SWI/SNF complexes, including SMARCB1, BRG1, BAF155, BAF170, ARID1A (BAF250A), PBRM1 (BAF180), and core histones. Regarding these conditions, we propose a model in which IPMK physically associates with SWI/SNF complex by directly binding to SMARCB1, BRG1, and BAF155 (Figure 7A, right). Our proposed model also suggests that IPMK may be positioned near the nucleosomal DNA exit site of SWI/SNF complex, which close to the Rpt1 domain of SMARCB1 (Figure 7-figure supplement 1).

It is previously suggested that the Rpt1 domain of SMARCB1 may facilitate the DNA detachment of the SWI/SNF complex, acting as a "wedge" at nucleosomal DNA exit sites (He et al., 2020). According to our results, IPMK is proximal to the core histones and it directly binds to the core subunits (BRG1 and Rpt1 domain of SMARCB1) that sandwich the nucleosomes, suggesting that IPMK may have a role in the interaction between SWI/SNF complex and chromatin. In support of this, our CUT\&RUN and ATAC-seq results indicate that the IPMK depletion disrupts the global BRG1 occupancy and its downstream, BRG1mediated, chromatin accessibility in a genome-wide manner, including promoter-TSS regions. Collectively, our findings suggest that IPMK plays an important role in regulating the localization of mammalian SWI/SNF complex by enhancing the SWI/SNF-nucleosome interactions via physical binding, thereby maintaining the appropriate chromatin accessibility (Figure 7B). Thus, when IPMK is depleted, it affects the SWI/SNF-Nucleosome interactions, resulting in perturbed BRG1 localizations and decreased chromatin accessibility (Figure 7B).

517 Despite our findings, the mechanism on how IPMK modulates the localization of BRG1 (or SWI/SNF 518 complex) remains unclear. We believe that the physical association of the IPMK-SWI/SNF complex is strongly connected with the BRG1 localization, but the detailed mechanism is still elusive. IPMK may

520 facilitate the recruitment of the SWI/SNF complex's subunits from cytoplasm to chromatin. However, our 521 observations from chromatin fractionation assays indicated that IPMK depletion did not affect the BRG1 522 or SMARCB1 occupancy in the cytoplasm or chromatin fractions (Figure 4A), excluding the above 523 possibility. Alternatively, it is plausible that IPMK may aid the appropriate conformation of specific subsets 524 of SWI/SNF complex by physically binding near the nucleosomal DNA exit sites within the SWI/SNF 525 complex. Thus, by aiding the appropriate conformation of the SWI/SNF complex, IPMK plays a vital role 526 in SWI/SNF-nucleosome interactions, thereby facilitating/stabilizing the BRG1 occupancy on the 527 chromatin. Although IPMK depletion reduced the global BRG1 occupancy, our CUT\&RUN results 528 indicated that IPMK depletion exhibits more impact at the CUT\&RUN peaks with lowly enriched BRG1 529 in EgfpKD (wild-type-like) mESCs, compared to highly enriched BRG1 peaks (Figure 4C and G-I). 530 Importantly, our ATAC-seq results also corresponded to the region-specific (BRG1 low) changes in BRG1 
occupancy upon IpmkKD (Figure 4C and G-I). In support of this, IPMK depletion exhibited more impact on the chromatin accessibility and transcription of the relatively-low-BRG1-harboring bivalent promoters than H3K4me3-Only promoters, which harbor high BRG1 levels (Figure 5 and 6). Together, these results

534 indicate that IPMK primarily regulates the BRG1 occupancy (which resembles the SWI/SNF-nucleosome interactions) and its downstream effects (chromatin accessibility and transcription) at the region where the BRG1 level is originally low in mESCs but does not affect the regions with high BRG1 level. This contextual discrepancy in IPMK-dependent or IPMK-independent BRG1 occupancy may provide key clues to the mechanism on how IPMK modulates the BRG1 localization, but further experiments/analyses are required.

540 Previous studies showing that IPMK regulates several target proteins (including cytosolic signaling factors and transcription factors) through protein-protein interaction also support our view. In earlier studies,

542 IPMK was found to bind to mTOR and raptor, maintaining the mTOR-raptor association and amino acid543 induced mTOR signaling (S. Kim et al., 2011). Glucose signaling activates the phosphorylation of IPMK, 544 enabling IPMK-AMPK binding, thereby enhancing its signaling (Bang et al., 2012). Furthermore, IPMK 545 stimulates $\mathrm{p} 53$-mediated transcription by binding to $\mathrm{p} 53$, thus facilitating $\mathrm{p} 53$-mediated cell death (Xu, Sen, 546 et al., 2013). IPMK also binds with CBP/p300, a transcriptional coactivator of CREB, and augments the 547 expression of CREB-regulated genes (Xu, Paul, et al., 2013). IPMK also binds to SRF, enhancing its 548 interactions with the serum response element at the promoters, and induces immediate early gene 549 expressions (E. Kim et al., 2013). Lastly, IPMK, which possesses PI3K activity, interacts with the SF-1 550 nuclear receptor and generates SF-1-PIP 3 complex, promoting lipid-mediated signaling in the nucleus 551 (Blind et al., 2014; Blind, Suzawa, \& Ingraham, 2012). Conversely, core subunits of the SWI/SNF complex 552 also interact with various factors, such as PDX1 and CTCF (Marino et al., 2019; Spaeth et al., 2019). In 553 addition, direct interactions between SWI/SNF complex and oncogenes/tumor-suppressor genes, such as 554 RB (Retinoblastoma), BRCA1, c-MYC, and MLL, have been implicated in oncogenesis (Roberts \& Orkin, 555 2004). Although these studies support our view that the direct physical association between IPMK and 556 SWI/SNF complex may affect diverse epigenetic events (such as BRG1 localization, chromatin 557 accessibility, or transcription), further experiments are required to elucidate the precise underlying 558 mechanisms of this phenomenon.

559 SWI/SNF chromatin remodeling complex is frequently mutated in cancer (Helming, Wang, \& Roberts, 560 2014). Particularly, SMARCB1 is a bona fide tumor suppressor gene (Roberts, Galusha, McMenamin, 561 Fletcher, \& Orkin, 2000; Roberts, Leroux, Fleming, \& Orkin, 2002), which is inactivated or lost in multiple 562 malignancies, such as malignant rhabdoid tumors. A germline mutation of IPMK was recently found in 563 familial small intestinal carcinoid patients (Sei et al., 2015). A truncated IPMK allele was associated with 564 reduced P53 signaling in these patients, suggesting a tumor suppressor role of IPMK. Additionally, the $565 I P M K$ mRNA levels were down-regulated by the tumor suppressor miR-18a, which contributes to the 566 inhibition of ovarian tumor growth (Liu et al., 2017). Finally, the exogenous supply of $\mathrm{IP}_{4}$, which is 567 produced by IPMK, can suppress human cancer cell growth by inhibiting the activation of AKT/PKB 568 (Jackson, Al-Saigh, Schultz, \& Junop, 2011; Piccolo et al., 2004; Razzini et al., 2000). Considering these 
569 previous reports with our results (that elucidated the physical/function linkage between the tumor

570 suppressor SMARCB1 and IPMK), it would be interesting to conduct genome-wide studies characterizing

571 the role of combined action of IPMK-SMARCB1 in the context of cancer.

572 Our study is the first to elucidate the physical association between IPMK and core subunits of SWI/SNF

573 complex, and the first to define the molecular function of IPMK in coordinating the BRG1 localizations

574 and BRG1-associated chromatin accessibility in mESCs. Considering our results and recently published

575 cryo-EM-based structure of human SWI/SNF (BAF) complex, we propose a model in which IPMK

576 physically associates with SWI/SNF complex via directly binding to SMARCB1, BRG1, and BAF155 and

577 positions near the nucleosomal DNA exit site of SWI/SNF complex, which is in close proximity to the Rpt1

578 domain of SMARCB1 (the IPMK-binding domain). Based on this model, IPMK plays an important role in

579 regulating the SWI/SNF-nucleosome interactions, thereby maintaining an appropriate BRG1 occupancy

580 and BRG1-mediated chromatin accessibility. We believe these novel findings will play a pivotal role in

581 future studies of IPMK and understanding the molecular mechanisms of mammalian SWI/SNF complexes,

582 especially by providing additional clues in SWI/SNF-mediated generation of nucleosome free regions at

583 the transcription start sites (TSS).

584 


\section{MATERIALS AND METHODS}

\section{Yeast two-hybrid screening.}

587 Panbionet (Pohang, South Korea) conducted yeast two-hybrid screening (http://panbionet.com). The full

588 IPMK coding region of 416 amino acids was amplified by polymerase chain reaction (PCR). The PCR

589 product was cloned into the pGBKT7 vector, which contains the DNA-binding domain (BD) of GAL4.

590 Saccharomyces cerevisiae strain AH109 (Clontech) was co-transformed with GAL4 DNA-BD-fused IPMK

591 and a human brain cDNA activation domain (AD) library (Clontech). Two different reporter genes (HIS3

592 and $A D E 2$ ) were used as selection markers. Yeast transformants were spread on a selection medium lacking

593 leucine, tryptophan, and adenine or histidine (SD-LWA and SD-LWH). To confirm the interactions, the

594 candidate prey genes of candidates were amplified via PCR or E. coli transformation, and reintroduced into

595 the AH109 yeast strain with the IPMK bait plasmid.

596

\section{Generation of stable cell lines for APEX2-mediated proximity labeling}

598 V5-APEX2 was PCR-amplified from the pcDNA5-Mito-V5-APEX2 plasmid, which was kindly provided

599 by Dr. Hyun-Woo Rhee (Seoul National University). V5-APEX2 alone or IPMK-V5-APEX2 were cloned

600 into the pcDNA ${ }^{\mathrm{TM}}$ /FRT/TO plasmid (Invitrogen). Flp-In ${ }^{\mathrm{TM}}$ T-REx ${ }^{\mathrm{TM}}-293$ (Invitrogen) cells were seeded

601 in 6-well culture plate to reach $70 \%$ confluency, then co-transfected with $0.25 \mu \mathrm{g}$ of pcDNA ${ }^{\mathrm{TM}} 5 / \mathrm{FRT} / \mathrm{TO}$

602 and $2.25 \mu \mathrm{g}$ of pOG44 Flp recombinase expression plasmid (Invitrogen) using Lipofectamine LTX with

603 Plus Reagent (Invitrogen). After 48 hours, the cells were transferred to $90 \mathrm{~mm}$ culture dishes to undergo

604 negative selection with $50 \mu \mathrm{g} / \mathrm{ml}$ Hygromycin B (Gibco) until all non-transfected cells were dead.

605 Surviving cells were then seeded with low confluency to generate cellular clones on culture plates, after

606 which each clone was individually screened for APEX2 construct expression with or without doxycycline

607 (Sigma Aldrich) in order to search for optimal cell populations with minimal uncontrolled APEX2

608 expression and maximal APEX2 expression under stimulation. Selected clones were then expanded and

609 stored in liquid nitrogen for downstream experiments.

610

\section{APEX2-mediated proximity labeling}

$6121.4 \times 10^{7} \mathrm{APEX} 2$-expressing cells were seeded in T75 culture flasks. 16 hours after seeding, the culture 613 medium was exchanged with complete medium supplemented with doxycycline $(100 \mathrm{ng} / \mathrm{ml})$ for APEX2 614 expression. After 24 hours of induction, the cells were incubated in fresh media containing $250 \mu \mathrm{M}$ 615 desthiobiotin-phenol (DBP) for 30 minutes in a $\mathrm{CO}_{2}$ incubator at $37^{\circ} \mathrm{C}$. The cells were then moved to room 616 temperature, and hydrogen peroxide (diluted in DPBS to $1 \mathrm{mM}$; Sigma Aldrich) was added to initiate the 617 APEX2-driven biotinylation reaction. The reaction was quenched by adding a $2 X$ quenching solution (20 618 M sodium ascorbate, $10 \mathrm{mM}$ Trolox, and $20 \mathrm{mM}$ sodium azide in DPBS) to the medium. The cells were 619 further washed with $1 \mathrm{X}$ quencher solution for three times, collected by centrifugation, snap-frozen and 620 stored at $-80^{\circ} \mathrm{C}$ until lysis. DBP was synthesized as described in a previous report (S. Y. Lee et al., 2017). 


\section{Preparation of DBP-labeled peptides for LC-MS/MS}

623 DBP-labeled peptides were prepared from frozen cell pellets as described in a previous report (Kwak et al.,

624 2020). Briefly, cells were lysed in lysis buffer (2\% SDS, 1 X protease inhibitor cocktail (Roche), and $1 \mathrm{mM}$

625 sodium azide in $1 \mathrm{X}$ TBS) and excess DBP was eliminated through repeated acetone precipitation. The

626 resulting protein precipitates were again solubilized in $50 \mathrm{mM}$ ammonium bicarbonate and quantified. 4

$627 \mathrm{mg}$ of cellular protein was then denatured, reduced, alkylated, and digested into peptides with trypsin.

628 Afterward, tryptic DBP-labeled peptides were bound to streptavidin beads (Pierce) and collected with

629 elution buffer ( $80 \%$ acetronitrile, $0.2 \%$ trifluoroacetic acid, $0.1 \%$ formic acid in MS-grade water). Solvents

630 were completely evaporated on a SpeedVac for 3 hours, and the resulting peptides were stored at $-20^{\circ} \mathrm{C}$

631 until required for LC-MS/MS analysis.

632

633 LC-MS/MS

634 The resulting tryptic peptides were analyzed by LC-MS/MS. All mass analyses were performed on a Q

635 Exactive Plus orbitrap mass spectrometer (Thermo Fisher Scientific) equipped with a nanoelectrospray ion

636 source. To separate the peptide mixture, we used a C18 reverse-phase HPLC column $(500 \mathrm{~mm} \times 75 \mu \mathrm{m} \mathrm{ID)}$

637 using an acetonitrile $/ 0.1 \%$ formic acid gradient from 3.2 to $26 \%$ for 120 minutes at a flow rate of 300

$638 \mathrm{~nL} / \mathrm{min}$. For MS/MS analysis, the precursor ion scan MS spectra $(\mathrm{m} / \mathrm{z} 400 \sim 2000)$ were acquired in the

639 Orbitrap at a resolution of 70,000 at $\mathrm{m} / \mathrm{z} 400$ with an internal lock mass. The 15 most intensive ions were

640 isolated and fragmented by High-energy collision induced dissociation (HCD).

641

642 LC-MS/MS data processing

643 All MS/MS samples were analyzed using Sequest Sorcerer platform (Sagen-N Research, San Jose, CA).

644 Sequest was set up to search the Homo sapiens protein sequence database (20675 entries, UniProt

645 (http://www.uniprot. org/)), which includes frequently observed contaminants, assuming the digestion

646 enzyme trypsin. Sequest was searched with a fragment ion mass tolerance of $1.00 \mathrm{Da}$ and a parent ion

647 tolerance of $10.0 \mathrm{ppm}$. Carbamidomethylation of cysteine was specified in Sequest as a fixed modification.

648 Oxidation of methionine and acetyl of the $\mathrm{n}$-terminus, biotin of lysine and DBP of tyrosine were specified

649 in Sequest as variable modifications. Scaffold (Version 4.11.0, Proteome Software Inc., Portland, OR) was

650 used to validate MS/MS-based peptide and protein identifications. Peptide identifications were accepted if

651 they could be established at greater than $93.0 \%$ probability to achieve a false discovery rate (FDR) less than

$6521.0 \%$ by the Scaffold Local FDR algorithm. Protein identifications were accepted if they could be

653 established at greater than $92.0 \%$ probability to achieve an FDR less than $1.0 \%$ and contained at least two

654 identified peptides. Protein probabilities were assigned by the Protein Prophet algorithm (Nesvizhskii,

655 Keller, Kolker, \& Aebersold, 2003). Proteins that contained similar peptides and could not be differentiated

656 based on MS/MS analysis alone were grouped to satisfy the principles of parsimony. Proteins were

657 annotated with GO terms from NCBI (downloaded November 23, 2019) (Ashburner et al., 2000).

658

659 Plasmids 
660 The cDNAs for human IPMK (NCBI Gene ID 253430) and human SMARCB1 (NCBI Gene ID 6598) were

661 obtained respectively from Open Biosystems and Bioneer (Daejeon, South Korea). IPMK and SMARCB1

662 cDNA constructs were amplified by PCR and the products were cloned into pCMV-GST and pcDNA3.1-

663 FLAG vectors. Every construct was confirmed by DNA sequencing.

664

\section{In vitro binding assay}

666 Recombinant human IPMK was purified as described previously (B. Lee et al., 2020). Briefly, human IPMK 667 was expressed in Sf9 insect cells with a baculovirus system, harvested with lysis buffer consisting of 300 $668 \mathrm{mM} \mathrm{NaCl}, 50 \mathrm{mM}$ Tris, $\mathrm{pH}$ 8.0, 5\% glycerol and $1 \mathrm{mM}$ phenylmethylsulfonylfluoride (PMSF). Freezing 669 and thawing lysis method with liquid nitrogen was applied to the cells, and the supernatants were taken 670 after centrifugation at 18,000 rpm for 90 minutes. Ni-NTA agarose (Qiagen) was applied with $20 \mathrm{mM}$ 671 imidazole incubated for 2 hours. The protein was eluted with $100 \mathrm{mM}$ imidazole and the N-terminal HIS672 tag was removed with TEV protease, followed by further purification with HiTrap and Superdex columns 673 (GE Healthcare). Human SMARCB1 was translated in vitro using TNT Quick Coupled 674 Transcription/Translation System (L1170, Promega). $1 \mu \mathrm{g}$ of pcDNA3.1-FLAG-SMARCB1 was incubated 675 at $30^{\circ} \mathrm{C}$ for 90 minutes with $20 \mu \mathrm{M}$ methionine and TNT T7 Quick Master Mix. Translated FLAG676 SMARCB1 was incubated with anti-FLAG M2 affinity gel (A2220, Sigma Aldrich), and then IPMK protein 677 was added and incubated with rotation at $4^{\circ} \mathrm{C}$.

678

\section{Recombinant IPMK protein purification}

680 For GST-tagged protein, human IPMK cDNA was subcloned into pGEX4T plasmid (Sigma Aldrich), 681 expressed in Escherichia coli, and purified on Glutathione Sepharose 4B beads (GE Healthcare) as 682 described (J. Kim \& Roeder, 2011). For FLAG-tagged proteins, wild-type or mutant IPMK cDNAs were 683 subcloned into pFASTBAC1 plasmid (Thermo Fisher Scientific) with an N-terminal FLAG epitope, and 684 baculoviruses were generated according to the manufacturer's instructions. Proteins were expressed in Sf9 685 insect cells and purified on M2 agarose (Sigma Aldrich) as described (J. Kim \& Roeder, 2011).

686

\section{BAF complex purification}

688 The FLAG-DPF2 cell line was selected from HEK293T cells transfected with a FLAG-DPF2-pCAG-IP 689 plasmid. Derived nuclear extracts (Dignam, Lebovitz, \& Roeder, 1983) were incubated with M2 agarose in 690 binding buffer (20 mM Tris $\mathrm{HCl}$ [pH 7.3], $300 \mathrm{mM} \mathrm{KCl}, 0.2 \mathrm{mM}$ EDTA, 25\% glycerol, $1.5 \mathrm{mM} \mathrm{MgCl}_{2}, 10$ $691 \mathrm{mM}$ 2-mercaptoethanol. and $0.2 \mathrm{mM} \mathrm{PMSF}$ ) at $4^{\circ} \mathrm{C}$ for $4 \mathrm{~h}$. After extensive washing with wash buffer (20 $692 \mathrm{mM}$ Tris $\mathrm{HCl}$ [pH 7.9], $150 \mathrm{mM} \mathrm{NaCl}, 0.2 \mathrm{mM}$ EDTA, 5\% glycerol, $2 \mathrm{mM} \mathrm{MgCl} 2,10 \mathrm{mM} \mathrm{2-}$ 693 mercaptoethanol, $0.2 \mathrm{mM}$ PMSF, and 0.1\% NP-40), complexes were eluted with wash buffer containing $6940.25 \mathrm{mg} / \mathrm{ml}$ FLAG peptide. Eluted complexes were fractionated by $10 \%-30 \%$ glycerol gradient and the 695 fractions containing intact BAF complex were combined and concentrated using Amicon Ultra-4 696 centrifugal filter (Millipore). 
699 For GST pull-down assays, 2ug of GST or GST-tagged IPMK immobilized on Glutathione Sepharose 4B

700 beads were incubated with $200 \mathrm{ng}$ of purified BAF complexes in binding buffer (20 mM Tris- $\mathrm{HCl}$ [pH 7.9],

$701150 \mathrm{mM} \mathrm{KCl}, 0.2 \mathrm{mM}$ EDTA, 20\% glycerol, $0.05 \% \mathrm{NP}-40$, and $0.2 \mathrm{mg} / \mathrm{ml} \mathrm{BSA}$ ) at $4{ }^{\circ} \mathrm{C}$ for $3 \mathrm{~h}$. Beads

702 were extensively washed with binding buffer without BSA, and bound proteins were analyzed by

703 immunoblotting. For binary protein interaction assays following baculovirus-mediated expression, $\mathrm{Sf} 9$ cells

704 were infected with baculoviruses expressing FLAG-IPMK and untagged BAF complex subunit. After 2

705 days, total cell extracts were prepared by sonication in lysis buffer (20 mM Tris-HCl [pH7.9], $300 \mathrm{mM}$

$706 \mathrm{NaCl}, 0.2 \mathrm{mM}$ EDTA, $15 \%$ glycerol, $2 \mathrm{mM} \mathrm{MgCl}$, $1 \mathrm{mM}$ DTT, $1 \mathrm{mM}$ PMSF, and protease inhibitor

707 cocktail [Roche]). Following clarification by centrifugation, cell extracts were incubated with $\mathrm{M} 2$ agarose

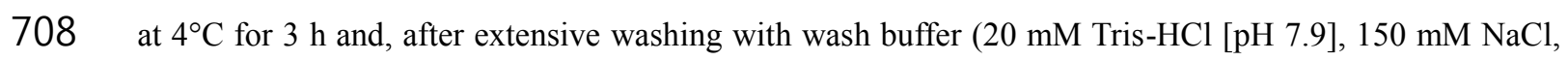

$7090.2 \mathrm{mM}$ EDTA, $15 \%$ glycerol, $2 \mathrm{mM} \mathrm{MgCl}, 1 \mathrm{mM} \mathrm{DTT}, 1 \mathrm{mM} \mathrm{PMSF}$, and $0.1 \% \mathrm{NP}-40$ ), bound proteins

710 were analyzed by immunoblotting.

711

712 Immunoblotting, immunoprecipitation, and GST pull-down

713 For immunoblot analyses, the cells were washed twice with PBS and lysed in lysis buffer consisting of $1 \%$

714 Triton X-100, $120 \mathrm{mM} \mathrm{NaCl}, 40 \mathrm{mM}$ Tris-HCl, $\mathrm{pH}$ 7.4, $1.5 \mathrm{mM}$ sodium orthovanadate, $50 \mathrm{mM}$ sodium

715 fluoride, $10 \mathrm{mM}$ sodium pyrophosphate, $1 \mathrm{mM}$ EDTA, and protease inhibitor cocktail (Roche). Cell lysates

716 were incubated at $4{ }^{\circ} \mathrm{C}$ for 10 minutes, and the supernatants were collected by centrifuging at $13,000 \mathrm{rpm}$

717 for $10 \mathrm{~min}$. Protein concentrations were determined by the Bradford protein assay (Bio-Rad) or

718 bicinchoninic acid (BCA) assay (Thermo Fisher Scientific). $20 \mu \mathrm{g}$ of protein lysates were separated by size,

719 transferred to nitrocellulose membranes, and blotted with primary antibodies and secondary antibodies. The

720 horseradish peroxidase (HRP) signals were visualized with the Clarity ECL substrate (Bio-Rad) and

721 SuperSignal ${ }^{\mathrm{TM}}$ West Femto Maximum Sensitivity Substrate (Thermo Fisher Scientific), and measured by

722 using a ChemiDoc imaging system (Bio-Rad). For immunoprecipitation, $2 \mathrm{mg}$ of total protein was

723 incubated with $5 \mu \mathrm{g}$ of primary antibodies for 16 hours with rotation at $4^{\circ} \mathrm{C} .10 \mu \mathrm{L}$ of TrueBlot beads

724 (Rockland Immunochemicals) were added and incubated for an additional hour. The samples were washed

725 three times with lysis buffer and prepared for immunoblotting. For GST pull-down assay, $10 \mu \mathrm{L}$ of

726 glutathione agarose beads (Incospharm) were added to $2 \mathrm{mg}$ of total cell lysates and incubated for 16 hours

727 with rotation at $4{ }^{\circ} \mathrm{C}$. The samples were then washed three times with lysis buffer and prepared for

728 immunoblotting.

729

\section{Cell culture and cell line production}

731 E14Tg2a mouse embryonic stem cells (mESCs) were maintained under feeder-free conditions. Briefly, the 732 cells were cultured on gelatin-coated cell culture dishes in an mESCs culture medium consisting of

733 Glasgow's minimum essential medium (GMEM) containing 10\% knockout serum replacement, 1\% non-

734 essential amino acids, $1 \%$ sodium pyruvate, $0.1 \mathrm{mM} \beta$-mercaptoethanol (all from Gibco), 1\% FBS, $0.5 \%$

735 antibiotic-antimycotic (both from Hyclone) and 1,000 units/ml LIF (ESG1106, Millipore). The mESCs 
were maintained at $37^{\circ} \mathrm{C}$ with $5 \% \mathrm{CO}_{2}$ in humidified air. NIH3T3 cells, mouse embryonic fibroblasts

737 (MEFs) and human embryonic kidney (HEK)-293T cells were grown in high-glucose DMEM

738 supplemented with 10\% FBS, $2 \mathrm{mM} \mathrm{L}$-glutamine, and penicillin/streptomycin $(100 \mathrm{mg} / \mathrm{ml})$, and maintained

739 in a humid atmosphere of $5 \% \mathrm{CO}_{2}$ at $37^{\circ} \mathrm{C}$. To generate tamoxifen-inducible IPMK knockout mice, Ipmk $k^{\text {flfl }}$

740 mice were mated with UBC-Cre-ERT2 mice (The Jackson Laboratory). The MEFs were immortalized by

741 transfecting with an SV40 large T-antigen plasmid, and the IPMK depletion was achieved by adding $1 \mu \mathrm{M}$

742 4-hydroxytamoxifen for 48 hours. FLAG epitope-tagged mESCs and MEFs were generated as described

743 previously (Savic et al., 2015). Briefly, the 3xFlag-P2A-Puromycin epitope tagging donor construct

744 (pFETCh-Donor), CRISPR guide RNAs (gRNAs), and Cas9 expressing plasmids were manufactured by

745 ToolGen (Seoul, Korea). mESCs were transfected using FUGENE HD (E2311, Promega), selected using

746 puromycin (A11138-03, Gibco), and expanded. MEFs were transfected with the donor construct containing

747 the neomycin resistance gene with Turbofect (R0533, Thermo Fisher Scientific) and selected using G418

748 (11811023, Gibco).

749

\section{RNA interference}

751 Control siRNA (scRNA) and siRNAs against Egfp (sense: 5'-GUUCAGCGUGUCCGGCGAG-3';

752 antisense: 5'-CUCGCCGGACACGCUGAAC-3') and Ipmk (5'-CAGAGAGGUCCUAGUUAAUUUCA-

753 3'; antisense: 5'-AGUGAAAUUAACUAGGACCUCUCUGUU-3') were synthesized and annealed by

754 Bioneer (Daejeon, Korea), and siRNA against Smarcb1 was purchased from Sigma Aldrich. mESCs and

755 MEFs were transfected with $50 \mathrm{nM}$ of the corresponding siRNA using DharmaFECT I (T-2001-03,

756 Dharmacon) according to the manufacturer's instructions. Briefly, the cells were seeded onto 6-well plates.

757 One day later, $50 \mathrm{nM}$ of siRNAs and DharmaFECT reagent were diluted in Opti-MEM (Gibco), incubated

758 separately at $25^{\circ} \mathrm{C}$ for 5 minutes, and then mixed together. The mixtures were incubated at $25^{\circ} \mathrm{C}$ for 20

759 minutes and added to the cell cultures. The culture medium was replaced after 24 hours and the transfected

760 cells were harvested at 48 hours after transfection.

761

\section{Chromatin fractionation}

763 A chromatin acid extraction was performed as described previously (Zhong, Martinez-Pastor, Silberman,

764 Sebastian, \& Mostoslavsky, 2013). mESCs or MEFs were collected and washed with PBS, and resuspended

765 with lysis buffer consisting of $10 \mathrm{mM}$ HEPES, pH 7.4, $10 \mathrm{mM} \mathrm{KCl}, 0.05 \% \mathrm{NP}-40,1 \mathrm{mM}$ sodium

766 orthovanadate, and protease inhibitor cocktail (Roche). The cell lysates were incubated 20 minutes on ice

767 and centrifuged at 13,000 rpm. The supernatant contained the cytoplasmic proteins, and the pellet with

768 nuclei was washed once with lysis buffer and centrifuged at 13,000 rpm for 10 minutes. The nuclei were

769 resuspended with low salt buffer consisting of $10 \mathrm{mM}$ Tris-HCl, $\mathrm{pH} 7.4,0.2 \mathrm{mM} \mathrm{MgCl}, 1 \%$ Triton-X 100,

$7701 \mathrm{mM}$ sodium orthovanadate, and protease inhibitor cocktail, then incubated 15 minutes on ice. After 10

771 minutes of centrifugation, the supernatant contained the nucleoplasmic proteins, and the pellet contained

772 the chromatin. The chromatin was then resuspended with $0.2 \mathrm{~N} \mathrm{HCl}$ for 20 minutes on ice, centrifuged at 
$77313,000 \mathrm{rpm}$ for 10 minutes, and neutralized with $1 \mathrm{M}$ Tris-HCl, $\mathrm{pH}$ 8.0. The protein concentrations were

774 determined and subjected to immunoblotting.

775

776 CUT\&RUN

777 CUT\&RUN assays were performed as previously described (Meers, Bryson, Henikoff, \& Henikoff, 2019;

778 Skene \& Henikoff, 2017), with minor modification. Briefly, 4 million mESCs were harvested and washed

779 with $1.5 \mathrm{ml}$ Wash buffer (20 mM HEPES, pH 7.5, $150 \mathrm{mM} \mathrm{NaCl}, 0.5 \mathrm{mM}$ Spermidine) three times. Cells

780 were bound to activated Concanavalin A-coated magnetic beads (at $25^{\circ} \mathrm{C}$ for 10 min on a nutator), then

781 permeabilized with Antibody buffer (Wash buffer containing 0.05\% Digitonin and 4 mM EDTA). The bead-

782 cell slurry was incubated with $3 \mu$ relevant antibody (see below) in a $150 \mu$ l volume at $25^{\circ} \mathrm{C}$ for 2 hours on

783 a nutator. After two washes in $1 \mathrm{ml}$ Dig-wash buffer (Wash buffer containing 0.05\% Digitonin), beads were

784 resuspended in $150 \mu \mathrm{l} \mathrm{pAG/MNase}$ and incubated at $4^{\circ} \mathrm{C}$ for 1 hour on a nutator. After two washes in $1 \mathrm{ml}$

785 Dig-wash buffer, beads were gently vortexed with $100 \mu \mathrm{l}$ Dig-wash buffer. Tubes were chilled to $0^{\circ} \mathrm{C}$ for

$7865 \mathrm{~min}$ and ice-cold $2.2 \mathrm{mM} \mathrm{CaCl} 2$ was added while gently vortexing. Tubes were immediately placed on

787 ice and incubated at $4{ }^{\circ} \mathrm{C}$ for 1 hour on a nutator, followed by addition of $100 \mu 12 \mathrm{xSTOP}$ buffer (340 mM

$788 \mathrm{NaCl}, 20 \mathrm{mM}$ EDTA, $4 \mathrm{mM}$ EGTA, 0.05\% Digitonin, $0.1 \mathrm{mg} / \mathrm{ml}$ RNase A, $50 \mu \mathrm{g} / \mathrm{ml}$ glycogen) and

789 incubated at $37^{\circ} \mathrm{C}$ for $30 \mathrm{~min}$ on a nutator. Beads were placed on a magnet stand and the liquid was removed

790 to a fresh tube, followed by addition of $2 \mu 110 \%$ SDS and $2.5 \mu l$ proteinase $\mathrm{K}(20 \mathrm{mg} / \mathrm{ml})$ and incubated at

$79150^{\circ} \mathrm{C}$ for 1 hour. DNA was extracted using phenol chloroform as described at

$792 \mathrm{https}: / / w w w . p r o t o c o l s . i o / v i e w / c u t-a m p-r u n-t a r g e t e d-i n-s i t u-g e n o m e-w i d e-p r o f i l i n g-z c p f 2 v n . ~ C U T \& R U N$

793 libraries were prepared using a NEXTflex ChIP-seq Library kit (5143-02, Bioo Scientific), according to

794 the manufacturer's guidelines. The libraries were then sequenced using an Illumina Novaseq 6000 platform.

795 The libraries were generated from two sets of biological replicates.

796

\section{$797 \quad$ Antibodies}

798 Antibodies against FLAG (F1804, Sigma Aldrich), IPMK (custom rabbit polyclonal antibody, raised 799 against a mouse IPMK peptide corresponding to amino acids 295-311 (SKAYSTHTKLYAKKHQS; 800 Covance)) (S. Kim et al., 2011), SMARCB1 (A301-087, Bethyl), BRG1 (ab110641, Abcam), BAF155 801 (11956, Cell Signaling Technology), BAF170 (12760, Cell Signaling Technology), BAF250A (12354, Cell 802 Signaling Technology), BRM (11966, Cell Signaling Technology), PBAF/PBRM (A301-591A, Bethyl), a803 TUBULIN (T5169, Sigma Aldrich), GST (2622, Cell Signaling Technology), LaminB1 (sc-365214, Santa 804 Cruz Biotech), Histone H3 (homemade), GAPDH (sc-32233, Santa Cruz Biotech), anti-DPF2 (ab128149, 805 Abcam), anti-SMARCE1 (ab137081, Abcam), anti-SS18L1 (ab227535, Abcam), anti-ACTL6A (sc806 137062, Santa Cruz Biotech), anti-SMARCD1 (sc-135843, Santa Cruz Biotech), anti-BCL7A (HPA019762, 807 Atlas Antibodies) and anti-ACTB (TA811000, Origene) were used for immunoblotting. Antibodies against 808 IPMK (homemade), SMARCB1 (A301-087, Bethyl) and normal rabbit IgG (sc-2027, Santa Cruz Biotech) 809 and anti-FLAG M2 affinity gel (A2220, Sigma Aldrich) were used for immunoprecipitation, and GST (2622, 
810 Cell Signaling Technology) was used for pulldown. Antibodies against FLAG (F7425, Sigma Aldrich),

811 BRG1 (ab110641, Abcam), and IgG (homemade) were used for CUT\&RUN assay.

812

813 ATAC-seq

814 ATAC-seq libraries were prepared as previously described (Buenrostro, Giresi, Zaba, Chang, \& Greenleaf,

815 2013; Buenrostro, Wu, Chang, \& Greenleaf, 2015), with minor modification. Briefly, 50,000 mESCs were

816 harvested, washed with cold PBS, lysed with cold lysis buffer, and immediately centrifuged. The nuclear

817 pellets were resuspended in $25 \mu$ of $2 \mathrm{X}$ tagmentation reaction buffer (10 mM Tris, $\mathrm{pH} 8.0,5 \mathrm{mM} \mathrm{MgCl}$,

$81810 \%$ dimethylformamide), $23 \mu \mathrm{l}$ of nuclease-free water, and $2 \mu \mathrm{l}$ of $\mathrm{Tn} 5$ transposase (in-house generated),

819 and incubated at $37^{\circ} \mathrm{C}$ for $30 \mathrm{~min}$. The samples were then immediately purified using a QIAquick PCR

820 purification kit (28106, Qiagen). The libraries were pre-enriched for five cycles using the KAPA HiFi

821 Hotstart ready mix (KK2601, Kapa Biosystems), and the threshold cycle (Ct) was monitored using qPCR

822 to determine the additional enrichment cycles, which were then applied. The final libraries were purified

823 again with a QIAquick PCR purification kit, and sequenced using an Illumina Novaseq 6000 platform. The

824 libraries were generated from two sets of biological replicates.

825

\section{H3K4me3-Only and bivalent promoter-TSS}

827 The list of 47,382 mouse genes was obtained from the UCSC genome browser (Table browser, mm10,

828 group: Genes and Gene Prediction, track: NCBI RefSeq, table: UCSC RefSeq (refGene), region: genome).

829 Among these genes, we selected protein-coding genes (gene name starting with NM_) that are longer than

$8302 \mathrm{~kb}$. To classify the promoter-TSS regions precisely, we removed redundancies by merging the genes,

831 having the exact same transcription start sites (TSS), into the same group. By doing so, we obtained 23,927

832 mouse promoter-TSS regions. To categorize the promoter-TSS regions depending on their histone

833 modifications status, we first calculated the H3K4me3 (accession number: GSM254000 and GSM254001)

834 and H3K27me3 (accession number: GSM254004 and GSM254005) ChIP-seq intensity in a $-500 /+1,000$

835 bp window around the promoter-TSS regions. The $-500 /+1,000 \mathrm{bp}$ window range was also applied in a

836 previous study (de Dieuleveult et al., 2016). Next, we divided the promoter-TSS into two groups based on

837 the first quartile (Q1) value of H3K4me3 ChIP-seq intensity at the whole promoter-TSS regions;

838 H3K4me3-Low and H3K4me3-High. We then divided the H3K4me3-High promoter-TSS into two groups

839 based on the third quartile (Q3) value of H3K27me3 ChIP-seq intensity at the whole promoter-TSS regions;

840 H3K4me3-Only and bivalent. Thus, we categorized three types of promoter-TSS; 5,982 H3K4me3-Low,

841 12,305 H3K4me3-Only (high H3K4me3 and low H3K27me3), and 5,640 bivalent (high H3K4me3 and

842 high H3K27me3). We obtained similar results when using the publically released H3K4me3 and

843 H3K27me3 ChIP-seq data (ENCODE, Ross Hardison, ENCSR212KGS and ENCSR059MBO).

844 Furthermore, we confirmed that the percentage of three promoter-TSS types are similar to the results

845 obtained by a previous study (de Dieuleveult et al., 2016).

847 mRNA purification and mRNA-seq 
Total RNA was purified from mESCs using the TRIzol reagent (Invitrogen) according to the manufacturer's instructions. Briefly, mESCs cultured in 6-well plates were harvested and homogenized with $1 \mathrm{ml}$ of TRIzol reagent. Chloroform ( $200 \mu \mathrm{L} / \mathrm{sample})$ was then added, and the samples were vigorously mixed by hand for 15 seconds and incubated at $25^{\circ} \mathrm{C}$ for 2 minutes. The mixtures were centrifuged at $12,000 \mathrm{rpm}$ for 15 minutes at $4^{\circ} \mathrm{C}$, and $500 \mu \mathrm{L}$ of each aqueous phase was transferred to a new Eppendorf tube and mixed with the equal volumes of isopropanol. The mixtures were incubated at $25^{\circ} \mathrm{C}$ for 10 minutes to precipitate the total RNA samples. The samples were then centrifuged at $12,000 \mathrm{rpm}$ for 10 minutes at $4{ }^{\circ} \mathrm{C}$, washed with $75 \%$ ethanol, and centrifuged again at $10,000 \mathrm{rpm}$ for 5 minutes at $4^{\circ} \mathrm{C}$. The RNA pellets were dried and dissolved in RNase-free water. For mRNA sequencing (mRNA-seq) library preparation, mRNA was isolated from total RNA using a Magnetic mRNA isolation kit (S1550S, NEB), and libraries were prepared using a NEXTflex Rapid directional RNA-seq kit (5138-08, Bioo Scientific). The libraries were sequenced using an Illumina HiSeq 2500 system. The libraries were generated from two sets of biological replicates.

\section{Gene Ontology analysis}

862 ConsensusPathDB was used to identify gene ontology terms associated with differentially expressed genes (DEGs) (Herwig et al., 2016).

\section{Data processing and analysis}

866 For CUT\&RUN analysis, raw reads were aligned to the mouse genome (mm10) using Bowtie2 (version

867 2.2.9) (Langmead \& Salzberg, 2012) with the parameter (--trim3 125 --local --very-sensitive-local --no868 unal --no-mixed --no-discordant -q --phred33 -I 10 -X 700), as the previous study (GSM2247138). For 869 ATAC-seq analysis, raw reads were aligned to the mouse genome (mm10) using Bowtie2 (version 2.2.9) 870 with the following parameter; --very-sensitive -X 100 -local. For mRNA-seq analysis, raw reads were 871 aligned to the mouse genome (mm10) using STAR (version 2.5.2a) with default parameters (Dobin et al., 872 2013). Generally, we used MACS2 (Zhang et al., 2008) to convert the aligned BAM files into bedGraph 873 files, and normalized the data with respect to the total read counts. Then, we used bedGraphToBigWig 874 (Kent, Zweig, Barber, Hinrichs, \& Karolchik, 2010) to convert the bedGraph files into bigWig files. The 875 bigWig files were used as input files for bwtool (Pohl \& Beato, 2014) (matrix and aggregate option) to 876 quantify the intensity (e.g., heatmaps or average line plots) of the relevant sequencing data. All of our raw 877 data (fastq files) were confirmed to be of good quality using FastQC 878 (http://www.bioinformatics.babraham.ac.uk/projects/fastqc/). For our CUT\&RUN analysis, we used 879 MACS2 (callpeak option, P-value $<0.005$ ) to identify the peaks (or binding sites) of protein of interest by 880 using IgG as backgrounds. The CUT\&RUN data was also subjected to HOMER annotatPeaks.pl (Heinz et 881 al., 2010) to elucidate the genomic contents within BRG1/IPMK-binding sites. For our mRNA-seq analyses, 882 we used Cufflinks (Trapnell et al., 2010) (Cuffdiff option, fr-firststrand) to assess the expression levels and 883 identify DEGs. Box plots, volcano plots, and other plots were drawn with R (ggplot2) (Wickham, 2009) 884 and heatmaps were drawn with Java TreeView (Saldanha, 2004). The examples of our genome-wide data 885 were visualized using the Integrative Genomics Viewer (IGV) (Robinson et al., 2011). 


\section{Public data acquisition}

888 Publicly released ChIP-seq data were downloaded from the NCBI GEO DataSets database. These data were

889 downloaded as sra or fastq files, and sra files were converted to fastq files using the SRA Toolkit

890 (https://trace.ncbi.nlm.nih.gov/Traces/sra/sra.cgi?view=software). Both the public datasets and our data

891 were then analyzed using the same methods.

892 


\section{DATA AVAILABILITY}

894 The NGS data from this study have been submitted to the NCBI Gene Expression Omnibus (GEO)

895 (http://www.ncbi.nlm.nih.gov/geo) under accession GSE158525.

896

\section{ACKNOWLEDGEMENTS}

898 We thank members of the S.K. and D. L. laboratory for their helpful discussions.

899

900 FUNDING

901 This work was supported by TJ Park Science Fellowship of the POSCO TJ Park Foundation (to S.E.P.) and

902 the National Research Foundation of Korea [NRF-2018R1A5A1024261 to S.K. and D.L.].

903

\section{CONFLICT OF INTERESTS}

905 The authors have no potential conflicts of interest to disclose.

906 
Arnaud, O., Le Loarer, F., \& Tirode, F. (2018). BAFfling pathologies: Alterations of BAF complexes in cancer. Cancer Lett, 419, 266-279. doi:10.1016/j.canlet.2018.01.046

Ashburner, M., Ball, C. A., Blake, J. A., Botstein, D., Butler, H., Cherry, J. M., . . Sherlock, G. (2000). Gene ontology: tool for the unification of biology. The Gene Ontology Consortium. Nat Genet, 25(1), 25-29. doi:10.1038/75556

Bang, S., Kim, S., Dailey, M. J., Chen, Y., Moran, T. H., Snyder, S. H., \& Kim, S. F. (2012). AMPactivated protein kinase is physiologically regulated by inositol polyphosphate multikinase. Proc Natl Acad Sci U S A, 109(2), 616-620. doi:10.1073/pnas.1119751109

Bechet, J., Greenson, M., \& Wiame, J. M. (1970). Mutations affecting the repressibility of arginine biosynthetic enzymes in Saccharomyces cerevisiae. Eur J Biochem, 12(1), 31-39. doi:10.1111/j.1432-1033.1970.tb00817.x

Bercy, J., Dubois, E., \& Messenguy, F. (1987). Regulation of arginine metabolism in Saccharomyces cerevisiae: expression of the three ARGR regulatory genes and cellular localization of their products. Gene, 55(2-3), 277-285. doi:10.1016/0378-1119(87)90287-3

Blind, R. D. (2014). Disentangling biological signaling networks by dynamic coupling of signaling lipids to modifying enzymes. Adv Biol Regul, 54, 25-38. doi:10.1016/j.jbior.2013.09.015

Blind, R. D., Sablin, E. P., Kuchenbecker, K. M., Chiu, H. J., Deacon, A. M., Das, D., . . Ingraham, H. A. (2014). The signaling phospholipid PIP3 creates a new interaction surface on the nuclear receptor SF-1. Proc Natl Acad Sci U S A, 111(42), 15054-15059. doi:10.1073/pnas.1416740111

Blind, R. D., Suzawa, M., \& Ingraham, H. A. (2012). Direct modification and activation of a nuclear receptor-PIP 2 complex by the inositol lipid kinase IPMK. Sci Signal, 5(229), ra44. doi:10.1126/scisignal.2003111

Buenrostro, J. D., Giresi, P. G., Zaba, L. C., Chang, H. Y., \& Greenleaf, W. J. (2013). Transposition of native chromatin for fast and sensitive epigenomic profiling of open chromatin, DNA-binding proteins and nucleosome position. Nat Methods, 10(12), 1213-1218. doi:10.1038/nmeth.2688

Buenrostro, J. D., Wu, B., Chang, H. Y., \& Greenleaf, W. J. (2015). ATAC-seq: A Method for Assaying Chromatin Accessibility Genome-Wide. Curr Protoc Mol Biol, 109, 21.29.21-29. doi:10.1002/0471142727.mb2129s109

Burton, A., Azevedo, C., Andreassi, C., Riccio, A., \& Saiardi, A. (2013). Inositol pyrophosphates regulate JMJD2C-dependent histone demethylation. Proc Natl Acad Sci U S A, 110(47), 18970-18975. doi:10.1073/pnas.1309699110

Chakraborty, A., Kim, S., \& Snyder, S. H. (2011). Inositol pyrophosphates as mammalian cell signals. Sci Signal, 4(188), re1. doi:10.1126/scisignal.2001958

Christ, C., \& Tye, B. K. (1991). Functional domains of the yeast transcription/replication factor MCM1. Genes Dev, 5(5), 751-763. doi:10.1101/gad.5.5.751

de Dieuleveult, M., Yen, K., Hmitou, I., Depaux, A., Boussouar, F., Bou Dargham, D., . . Gerard, M. (2016). Genome-wide nucleosome specificity and function of chromatin remodellers in ES cells. Nature, 530(7588), 113-116. doi:10.1038/nature16505

Dignam, J. D., Lebovitz, R. M., \& Roeder, R. G. (1983). Accurate transcription initiation by RNA polymerase II in a soluble extract from isolated mammalian nuclei. Nucleic acids research, 11(5), 1475-1489. doi:10.1093/nar/11.5.1475

Dobin, A., Davis, C. A., Schlesinger, F., Drenkow, J., Zaleski, C., Jha, S., . . Gingeras, T. R. (2013). STAR: ultrafast universal RNA-seq aligner. Bioinformatics, 29(1), 15-21. doi:10.1093/bioinformatics/bts635

Dubois, E., Bercy, J., \& Messenguy, F. (1987). Characterization of two genes, ARGRI and ARGRIII required for specific regulation of arginine metabolism in yeast. Mol Gen Genet, 207(1), 142148. doi:10.1007/bf00331501

Hargreaves, D. C., \& Crabtree, G. R. (2011). ATP-dependent chromatin remodeling: genetics, genomics and mechanisms. Cell Res, 21(3), 396-420. doi:10.1038/cr.2011.32

Hatch, A. J., \& York, J. D. (2010). SnapShot: Inositol phosphates. Cell, 143(6), 1030-1030.e1031. doi:10.1016/j.cell.2010.11.045

He, S., Wu, Z., Tian, Y., Yu, Z., Yu, J., Wang, X., . . Xu, Y. (2020). Structure of nucleosome-bound human BAF complex. Science, 367(6480), 875-881. doi:10.1126/science.aaz9761

Heinz, S., Benner, C., Spann, N., Bertolino, E., Lin, Y. C., Laslo, P., . . Glass, C. K. (2010). Simple 
combinations of lineage-determining transcription factors prime cis-regulatory elements required for macrophage and B cell identities. Mol Cell, 38(4), 576-589. doi:10.1016/j.molcel.2010.05.004

Helming, K. C., Wang, X., \& Roberts, C. W. M. (2014). Vulnerabilities of mutant SWI/SNF complexes in cancer. Cancer Cell, 26(3), 309-317. doi:10.1016/j.ccr.2014.07.018

Herwig, R., Hardt, C., Lienhard, M., \& Kamburov, A. (2016). Analyzing and interpreting genome data at the network level with ConsensusPathDB. Nat Protoc, 11(10), 1889-1907. doi:10.1038/nprot.2016.117

Ho, L., Jothi, R., Ronan, J. L., Cui, K., Zhao, K., \& Crabtree, G. R. (2009). An embryonic stem cell chromatin remodeling complex, esBAF, is an essential component of the core pluripotency transcriptional network. Proc Natl Acad Sci US A, 106(13), 5187-5191. doi:10.1073/pnas.0812888106

Ho, L., Ronan, J. L., Wu, J., Staahl, B. T., Chen, L., Kuo, A., . . Crabtree, G. R. (2009). An embryonic stem cell chromatin remodeling complex, esBAF, is essential for embryonic stem cell selfrenewal and pluripotency. Proc Natl Acad Sci U S A, 106(13), 5181-5186. doi:10.1073/pnas.0812889106

Hodges, C., Kirkland, J. G., \& Crabtree, G. R. (2016). The Many Roles of BAF (mSWI/SNF) and PBAF Complexes in Cancer. Cold Spring Harb Perspect Med, 6(8). doi:10.1101/cshperspect.a026930

Jackson, S. G., Al-Saigh, S., Schultz, C., \& Junop, M. S. (2011). Inositol pentakisphosphate isomers bind $\mathrm{PH}$ domains with varying specificity and inhibit phosphoinositide interactions. BMC Struct Biol, 11, 11. doi:10.1186/1472-6807-11-11

Kent, W. J., Zweig, A. S., Barber, G., Hinrichs, A. S., \& Karolchik, D. (2010). BigWig and BigBed: enabling browsing of large distributed datasets. Bioinformatics, 26(17), 2204-2207. doi:10.1093/bioinformatics/btq351

Khavari, P. A., Peterson, C. L., Tamkun, J. W., Mendel, D. B., \& Crabtree, G. R. (1993). BRG1 contains a conserved domain of the SWI2/SNF2 family necessary for normal mitotic growth and transcription. Nature, 366(6451), 170-174. doi:10.1038/366170a0

Kim, E., Ahn, H., Kim, M. G., Lee, H., \& Kim, S. (2017). The Expanding Significance of Inositol Polyphosphate Multikinase as a Signaling Hub. Mol Cells, 40(5), 315-321. doi:10.14348/molcells.2017.0066

Kim, E., Beon, J., Lee, S., Park, S. J., Ahn, H., Kim, M. G., . . Kim, S. (2017). Inositol polyphosphate multikinase promotes Toll-like receptor-induced inflammation by stabilizing TRAF6. Sci Adv, 3(4), e1602296. doi:10.1126/sciadv.1602296

Kim, E., Tyagi, R., Lee, J. Y., Park, J., Kim, Y. R., Beon, J., . . Kim, S. (2013). Inositol polyphosphate multikinase is a coactivator for serum response factor-dependent induction of immediate early genes. Proc Natl Acad Sci U S A, 110(49), 19938-19943. doi:10.1073/pnas.1320171110

Kim, J., \& Roeder, R. G. (2011). Nucleosomal H2B ubiquitylation with purified factors. Methods (San Diego, Calif.), 54(3), 331-338. doi:10.1016/j.ymeth.2011.03.009

Kim, K. H., \& Roberts, C. W. (2014). Mechanisms by which SMARCB1 loss drives rhabdoid tumor growth. Cancer Genet, 207(9), 365-372. doi:10.1016/j.cancergen.2014.04.004

Kim, S., Kim, S. F., Maag, D., Maxwell, M. J., Resnick, A. C., Juluri, K. R., . . Snyder, S. H. (2011). Amino acid signaling to mTOR mediated by inositol polyphosphate multikinase. Cell Metab, 13(2), 215-221. doi:10.1016/j.cmet.2011.01.007

Kouzarides, T. (2007). Chromatin modifications and their function. Cell, 128(4), 693-705. doi:10.1016/j.cell.2007.02.005

Kwak, C., Shin, S., Park, J. S., Jung, M., Nhung, T. T. M., Kang, M. G., . . Rhee, H. W. (2020). ContactID, a tool for profiling organelle contact sites, reveals regulatory proteins of mitochondrialassociated membrane formation. Proc Natl Acad Sci U S A, 117(22), 12109-12120. doi:10.1073/pnas.1916584117

Langmead, B., \& Salzberg, S. L. (2012). Fast gapped-read alignment with Bowtie 2. Nat Methods, 9(4), 357-359. doi:10.1038/nmeth.1923

Lee, B., Park, S. J., Lee, S., Park, S. E., Lee, E., Song, J. J., . . Kim, S. (2020). Identification of the Antidepressant Vilazodone as an Inhibitor of Inositol Polyphosphate Multikinase by StructureBased Drug Repositioning. Mol Cells, 43(3), 222-227. doi:10.14348/molcells.2020.0051

Lee, S. Y., Kang, M. G., Shin, S., Kwak, C., Kwon, T., Seo, J. K., . . Rhee, H. W. (2017). Architecture Mapping of the Inner Mitochondrial Membrane Proteome by Chemical Tools in Live Cells. J Am Chem Soc, 139(10), 3651-3662. doi:10.1021/jacs.6b10418 
Liu, P., Qi, X., Bian, C., Yang, F., Lin, X., Zhou, S., .. . Yi, T. (2017). MicroRNA-18a inhibits ovarian cancer growth via directly targeting TRIAP1 and IPMK. Oncol Lett, 13(6), 4039-4046. doi:10.3892/ol.2017.5961

Maag, D., Maxwell, M. J., Hardesty, D. A., Boucher, K. L., Choudhari, N., Hanno, A. G., . . Resnick, A. C. (2011). Inositol polyphosphate multikinase is a physiologic PI3-kinase that activates Akt/PKB. Proceedings of the National Academy of Sciences, 108(4), 1391-1396. doi:10.1073/pnas.1017831108

Malabanan, M. M., \& Blind, R. D. (2016). Inositol polyphosphate multikinase (IPMK) in transcriptional regulation and nuclear inositide metabolism. Biochem Soc Trans, 44(1), 279-285. doi:10.1042/bst20150225

Marino, M. M., Rega, C., Russo, R., Valletta, M., Gentile, M. T., Esposito, S., . . Pedone, P. V. (2019). Interactome mapping defines BRG1, a component of the SWI/SNF chromatin remodeling complex, as a new partner of the transcriptional regulator CTCF. J Biol Chem, 294(3), 861-873. doi:10.1074/jbc.RA118.004882

Meers, M. P., Bryson, T. D., Henikoff, J. G., \& Henikoff, S. (2019). Improved CUT\&RUN chromatin profiling tools. Elife, 8. doi:10.7554/eLife.46314

Messenguy, F., \& Dubois, E. (1993). Genetic evidence for a role for MCM1 in the regulation of arginine metabolism in Saccharomyces cerevisiae. Mol Cell Biol, 13(4), 2586-2592. doi: $10.1128 / \mathrm{mcb} \cdot 13.4 .2586$

Nesvizhskii, A. I., Keller, A., Kolker, E., \& Aebersold, R. (2003). A statistical model for identifying proteins by tandem mass spectrometry. Anal Chem, 75(17), 4646-4658. doi:10.1021/ac0341261

Odom, A. R., Stahlberg, A., Wente, S. R., \& York, J. D. (2000). A role for nuclear inositol 1,4,5trisphosphate kinase in transcriptional control. Science, 287(5460), 2026-2029.

Piccolo, E., Vignati, S., Maffucci, T., Innominato, P. F., Riley, A. M., Potter, B. V., . . Falasca, M. (2004). Inositol pentakisphosphate promotes apoptosis through the PI 3-K/Akt pathway. Oncogene, 23(9), 1754-1765. doi:10.1038/sj.onc.1207296

Pohl, A., \& Beato, M. (2014). bwtool: a tool for bigWig files. Bioinformatics, 30(11), 1618-1619. doi:10.1093/bioinformatics/btu056

Razzini, G., Berrie, C. P., Vignati, S., Broggini, M., Mascetta, G., Brancaccio, A., \& Falasca, M. (2000). Novel functional PI 3-kinase antagonists inhibit cell growth and tumorigenicity in human cancer cell lines. Faseb j, 14(9), 1179-1187. doi:10.1096/fasebj.14.9.1179

Resnick, A. C., Snowman, A. M., Kang, B. N., Hurt, K. J., Snyder, S. H., \& Saiardi, A. (2005). Inositol polyphosphate multikinase is a nuclear PI3-kinase with transcriptional regulatory activity. Proc Natl Acad Sci U S A, 102(36), 12783-12788. doi:10.1073/pnas.0506184102

Roberts, C. W., Galusha, S. A., McMenamin, M. E., Fletcher, C. D., \& Orkin, S. H. (2000). Haploinsufficiency of Snf5 (integrase interactor 1) predisposes to malignant rhabdoid tumors in mice. Proc Natl Acad Sci U S A, 97(25), 13796-13800. doi:10.1073/pnas.250492697

Roberts, C. W., Leroux, M. M., Fleming, M. D., \& Orkin, S. H. (2002). Highly penetrant, rapid tumorigenesis through conditional inversion of the tumor suppressor gene Snf5. Cancer Cell, 2(5), 415-425. doi:10.1016/s1535-6108(02)00185-x

Roberts, C. W., \& Orkin, S. H. (2004). The SWI/SNF complex--chromatin and cancer. Nat Rev Cancer, 4(2), 133-142. doi:10.1038/nrc1273

Robinson, J. T., Thorvaldsdóttir, H., Winckler, W., Guttman, M., Lander, E. S., Getz, G., \& Mesirov, J. P. (2011). Integrative genomics viewer. Nat Biotechnol, 29(1), 24-26. doi:10.1038/nbt. 1754

Saiardi, A., Erdjument-Bromage, H., Snowman, A. M., Tempst, P., \& Snyder, S. H. (1999). Synthesis of diphosphoinositol pentakisphosphate by a newly identified family of higher inositol polyphosphate kinases. Curr Biol, 9(22), 1323-1326.

Saldanha, A. J. (2004). Java Treeview--extensible visualization of microarray data. Bioinformatics, 20(17), 3246-3248. doi:10.1093/bioinformatics/bth349

Savic, D., Partridge, E. C., Newberry, K. M., Smith, S. B., Meadows, S. K., Roberts, B. S., . . Myers, R. M. (2015). CETCh-seq: CRISPR epitope tagging ChIP-seq of DNA-binding proteins. Genome Res, 25(10), 1581-1589. doi:10.1101/gr.193540.115

Sei, Y., Zhao, X., Forbes, J., Szymczak, S., Li, Q., Trivedi, A., . . Wank, S. A. (2015). A Hereditary Form of Small Intestinal Carcinoid Associated With a Germline Mutation in Inositol Polyphosphate Multikinase. Gastroenterology, 149(1), 67-78. doi:10.1053/j.gastro.2015.04.008

Shen, X., Xiao, H., Ranallo, R., Wu, W.-H., \& Wu, C. (2003). Modulation of ATP-Dependent ChromatinRemodeling Complexes by Inositol Polyphosphates. Science, 299(5603), 112-114. 
Skene, P. J., \& Henikoff, S. (2017). An efficient targeted nuclease strategy for high-resolution mapping of DNA binding sites. Elife, 6. doi:10.7554/eLife.21856

Spaeth, J. M., Liu, J. H., Peters, D., Guo, M., Osipovich, A. B., Mohammadi, F., . . Stein, R. (2019). The Pdx1-Bound Swi/Snf Chromatin Remodeling Complex Regulates Pancreatic Progenitor Cell Proliferation and Mature Islet $\beta$-Cell Function. Diabetes, 68(9), 1806-1818. doi:10.2337/db190349

Steger, D. J., Haswell, E. S., Miller, A. L., Wente, S. R., \& O'Shea, E. K. (2003). Regulation of chromatin remodeling by inositol polyphosphates. Science, 299(5603), 114-116. doi:10.1126/science.1078062

Tolstorukov, M. Y., Sansam, C. G., Lu, P., Koellhoffer, E. C., Helming, K. C., Alver, B. H., . . Roberts, C. W. M. (2013). Swi/Snf chromatin remodeling/tumor suppressor complex establishes nucleosome occupancy at target promoters. Proceedings of the National Academy of Sciences, 110(25), 10165-10170. doi:10.1073/pnas.1302209110

Trapnell, C., Williams, B. A., Pertea, G., Mortazavi, A., Kwan, G., van Baren, M. J., . . Pachter, L. (2010). Transcript assembly and quantification by RNA-Seq reveals unannotated transcripts and isoform switching during cell differentiation. Nat Biotechnol, 28(5), 511-515. doi:10.1038/nbt.1621

Trotter, K. W., \& Archer, T. K. (2007). Nuclear receptors and chromatin remodeling machinery. Mol Cell Endocrinol, 265-266, 162-167. doi:10.1016/j.mce.2006.12.015

Vignali, M., Hassan, A. H., Neely, K. E., \& Workman, J. L. (2000). ATP-dependent chromatin-remodeling complexes. Mol Cell Biol, 20(6), 1899-1910. doi:10.1128/mcb.20.6.1899-1910.2000

Wang, W., Côté, J., Xue, Y., Zhou, S., Khavari, P. A., Biggar, S. R., . . Crabtree, G. R. (1996). Purification and biochemical heterogeneity of the mammalian SWI-SNF complex. Embo j, 15(19), 5370-5382.

Wang, W., Xue, Y., Zhou, S., Kuo, A., Cairns, B. R., \& Crabtree, G. R. (1996). Diversity and specialization of mammalian SWI/SNF complexes. Genes Dev, 10(17), 2117-2130. doi:10.1101/gad.10.17.2117

Wang, X., Haswell, J. R., \& Roberts, C. W. (2014). Molecular pathways: SWI/SNF (BAF) complexes are frequently mutated in cancer--mechanisms and potential therapeutic insights. Clin Cancer Res, 20(1), 21-27. doi:10.1158/1078-0432.Ccr-13-0280

Wickham, H. (2009). ggplot2: Elegant Graphics for Data Analysis: Springer-Verlag New York.

Xu, R., Paul, B. D., Smith, D. R., Tyagi, R., Rao, F., Khan, A. B., . . Snyder, S. H. (2013). Inositol polyphosphate multikinase is a transcriptional coactivator required for immediate early gene induction. Proc Natl Acad Sci U S A, 110(40), 16181-16186. doi:10.1073/pnas.1315551110

Xu, R., Sen, N., Paul, B. D., Snowman, A. M., Rao, F., Vandiver, M. S., . . Snyder, S. H. (2013). Inositol polyphosphate multikinase is a coactivator of p53-mediated transcription and cell death. Sci Signal, 6(269), ra22. doi:10.1126/scisignal.2003405

$\mathrm{Xu}, \mathrm{R} .$, \& Snyder, S. H. (2013). Gene transcription by p53 requires inositol polyphosphate multikinase as a co-activator. Cell Cycle, 12(12), 1819-1820. doi:10.4161/cc.25119

Zhang, Y., Liu, T., Meyer, C. A., Eeckhoute, J., Johnson, D. S., Bernstein, B. E., . . Liu, X. S. (2008). Model-based analysis of ChIP-Seq (MACS). Genome Biol, 9(9), R137. doi:10.1186/gb-2008-99-r137

Zhong, L., Martinez-Pastor, B., Silberman, D. M., Sebastian, C., \& Mostoslavsky, R. (2013). Assaying chromatin sirtuins. Methods Mol Biol, 1077, 149-163. doi:10.1007/978-1-62703-637-5_10 
TABLE AND FIGURES LEGENDS

Figure 1. Identification of SMARCB1 as an IPMK-interacting protein via unbiased screening assays. (A) IPMK and SMARCB1 interaction test in yeast strain AH109, containing two reporter genes (ADE2 and HIS3). Yeast cells were co-transformed with either the GAL4-BD fusion plasmid pGBKT7 or pGBKT7IPMK and the GAL4-AD fusion plasmid pACT2-SMARCB1. The yeast cells were spread on a selection medium lacking leucine and tryptophan (SD-LW) to select co-transformants of bait and prey vectors. Specific interactions between bait and prey proteins were monitored by cell growth on a selection medium lacking leucine, tryptophan, adenine (SD-LWA), or a selection medium lacking leucine, tryptophan, histidine (SD-LWH). 3-AT (3-amino-1,2,4-triazole) was used to suppress leaky HIS3 expression in transformants to obtain an accurate phenotype. Polypyrimidine tract binding protein (PTB) gene fused with the GAL4 DNA binding domain (BD-PTB) and PTB gene fused with the GAL4 activation domain (ADPTB) were used as positive controls of bait and prey vectors, respectively. The negative control is the cells transformed with parental bait vector (pGBKT7) and prey vector (pACT2). (B) A schematic diagram displaying identification strategy of IPMK-proximal/interacting proteins, which are biotinylated by APEX-tagged IPMK. (C) Bar graphs showing the relative abundance of biotinylated proteins related to SWI/SNF complex and two negative controls (left). Target proteins were arranged according to their significance $(P$-value, left: significant; right: not significant). A volcano plot showing the relative abundance and significance $(P$-value $)$ of biotinylated proteins related to SWI/SNF complex and two negative controls (right). A dotted line within the volcano plot indicates the $P$-value $=0.05$. The relative abundance (abundance ratio) was derived by comparing the fold enrichment of target proteins in IPMK-APEX2-expressed to APEX2-expressed HEK293 cells. $P$-value was calculated using Student's t-test.

Figure 2. IPMK binds to SMARCB1 and other components of the SWI/SNF complex.

1148 (A) Purified IPMK and in vitro translated FLAG-SMARCB1 were co-incubated, immunoprecipitated with 1149 FLAG antibody, and subjected to immunoblotting.

1150 (B) Sf9 insect cells were co-infected with baculoviruses expressing FLAG-IPMK and individual subunits 1151 of SWI/SNF complex (SMARCB1, BRG1, BAF155, and BAF170), followed by FLAG M2 agarose 1152 immunoprecipitation and immunoblotting.

1153 (C) IPMK and IgG were immunoprecipitated from E14Tg2a cells and subjected to immunoblotting.

1154 (D) SMARCB1 and IgG were immunoprecipitated from E14Tg2a cells and subjected to immunoblotting.

1155 (E) E14Tg2a cells were transfected with FLAG-IPMK or FLAG (a control vector), followed by FLAG 1156 immunoprecipitation and immunoblotting.

1157 (F) E14Tg2a cells were transfected with siRNA against Egfp (EgfpKD) and Ipmk (IpmkKD), 1158 immunoprecipitated with IPMK and IgG, and subjected to immunoblotting.

Figure 3. Identification of distinct domains required for IPMK-SMARCB1 interactions. 
1161 (A) A schematic diagram of the human SMARCB1 fragments used for the binding studies (B and C). The 1162 IPMK-binding sites (Rpt1 and Rpt2) are highlighted in red.

1163 (B) HEK293T cells were co-transfected with GST-IPMK and FLAG (a control vector) or FLAG-

1164 SMARCB1 fragments, followed by immunoprecipitation with FLAG antibody, and subjected to 1165 immunoblotting.

1166 (C and D) HEK293T cells were co-transfected with FLAG-IPMK and GST (a control vector) or GST-

1167 SMARCB1 fragments, followed by immunoprecipitation with FLAG antibody, and subjected to

1168 immunoblotting. The specific IPMK-binding SMARCB1 fragments are highlighted in bold.

1169 (E) A schematic diagram of the human SMARCB1 domains. SMARCB1 fragments used for the binding 1170 studies (D) are indicated below with the numbers of amino acid sequences. The specific IPMK-binding 1171 SMARCB1 fragments (Rpt1 and Rpt2) are highlighted in red.

1172 (F) HEK293T cells were co-transfected with FLAG-SMARCB1 and GST (a control vector) or GST-IPMK 1173 fragments, followed by immunoprecipitation with FLAG antibody, and subjected to immunoblotting.

1174 (G) A schematic diagram of human IPMK domains. IPMK fragments used for the binding studies (F) are 1175 indicated below with the numbers of amino acid sequences. Key domains for inositol binding (IP), kinase 1176 activity (SSLL and IDF), and nuclear localization signal (NLS) are depicted. The specific SMARCB11177 binding IPMK fragments (Exon3, 4, and 6) are highlighted in blue.

Figure 4. IPMK-BRG1 are co-localized at promoter-TSS, and IPMK regulates BRG1 localization.

1180 (A) E14Tg2a cells were transfected with siRNA against Egfp (EgfpKD) and Ipmk (IpmkKD) and then

1181 fractionated into the cytoplasm, nucleoplasm, and chromatin fractions. Immunoblotting with IPMK,

1182 SMARCB1, BRG1, and fractionation markers was then performed.

1183 (B) Examples of CUT\&RUN assays in E14Tg2a cells, including representative results for BRG1 (EgfpKD 1184 and IpmkKD), IPMK, and IgG. The BRG1 CUT\&RUN peak ( $E g f p$ KD cells) is marked as a blue box on 1185 top.

1186 (C) Heatmaps representing CUT\&RUN results for BRG1 (EgfpKD, IpmkKD, and their comparison), IPMK, 1187 and IgG at BRG1 CUT\&RUN peaks ( $E g f p K D$ cells) as indicated on top. All heatmaps were aligned at 118834,634 BRG1 CUT\&RUN peaks (rows) and sorted in descending order by the BRG1 intensity of EgfpKD 1189 cells.

1190 (D) Bar graphs showing the $\log _{2}$ enrichment of CUT\&RUN peaks (IPMK and BRG1-EgfpKD, -IpmkKD) 1191 annotated with various regions of the mouse genome.

1192 (E) Bar graphs showing the significance (Log $P$-value) of CUT\&RUN peaks (IPMK and BRG1-EgfpKD, 1193 -IpmkKD) annotated with various regions of the mouse genome. For each CUT\&RUN peak, genome 1194 annotations (e.g., promoters or CpG islands) are sorted in descending order according to their significance 1195 ( $P$-values, top-bottom, whereas top-more significant and bottom-less significant).

1196 (F) Examples of BRG1 (EgfpKD and IpmkKD), IPMK, IgG CUT\&RUN, and ATAC-seq (EgfpKD and 1197 IpmkKD) assays in E14Tg2a cells. The BRG1 CUT\&RUN peak (EgfpKD cells) and ATAC-seq peaks are 1198 marked as blue and green boxes on top. Major BRG1 peak (the most enriched site) and transcription start 
site (TSS) are indicated as black and red dotted lines.

1200 (G) Heatmaps representing BRG1 CUT\&RUN (EgfpKD, IpmkKD, and their comparison) at BRG1

1201 CUT\&RUN peaks ( $E g f p$ KD cells) assigned with nearby (within 2kb) ATAC-seq peaks (left). BRG1 peaks

1202 without nearby ATAC-seq peaks were excluded. In order to match the arrangement with ATAC-seq peaks

1203 (right), a BRG1 peak containing multiple ATAC-seq peaks was included without deduplication. Heatmaps

1204 representing ATAC-seq signals ( $E g f p \mathrm{KD}$, IpmkKD, and their comparison) at ATAC-seq peaks assigned with

1205 closest BRG1 CUT\&RUN peaks that were used for heatmaps on the left (right). All heatmaps were aligned

1206 at 36,481 BRG1 CUT\&RUN peaks (left) or 36,481 ATAC-seq peaks (right) and sorted in descending order

1207 by the BRG1 intensity of EgfpKD cells. High and Low groups were divided equally (n=18240 and 18241,

1208 respectively) according to the BRG1 intensity of $E g f p$ KD cells.

1209 (H) Box plots showing the differential BRG1 (left) and ATAC (right) intensity upon IpmkKD at High (grey)

1210 and Low (red) BRG1 CUT\&RUN peaks (left) and corresponding (closest) ATAC-seq peaks (right). High

1211 and Low groups ( $\mathrm{n}=18240$ and 18241 , respectively) were divided according to the BRG1 intensity of

1212 EgfpKD cells.

1213 (I) Box plots showing the differential BRG1 (left) and ATAC (right) intensity upon IpmkKD at High (grey)

1214 and Low (red) BRG1 CUT\&RUN peaks localized at Promoter-TSS (left) and corresponding (closest)

1215 ATAC-seq peaks (right). High and Low groups ( $\mathrm{n}=5,800$ and 2,618, respectively) were derived from (G

1216 and $\mathrm{H})$.

1217 (H and I) $P$-values were calculated using the Wilcoxon rank sum test.

1218

1219 Figure 5. IPMK affects chromatin accessibility at promoter-TSS by regulating the BRG1 localization.

1220 (A) Line plots showing the average enrichments of BRG1, nucleosome (MNase-seq, GSM5253962 and

1221 GSM5253963), and ATAC-seq signals (ATAC) at TSS of total genes (left) and TSS of three promoter types

1222 (right). Three genomic regions, indicated on top (see also dotted lines on left), were defined according to

1223 the relative position of enriched ATAC-seq intensity (Center, purple) and enriched BRG1 intensity

1224 (Upstream and Downstream, red and orange, respectively). Green, red, and blue lines on the right indicate

1225 H3K4me3-Low, H3K4me3-Only, and bivalent promoters, respectively.

1226 (B) Bar graphs showing the number of TSS (TSS exhibiting decreased BRG1 intensity upon IpmkKD) with

1227 different promoter types.

1228 (C) A diagram displaying five clusters of TSS classified by changes in BRG1 level at Up/Downstream

1229 regions (defined in (A)) upon IpmkKD (left). Arrows with downwards and upwards indicate decreased and

1230 increased BRG1 level upon IpmkKD, respectively. Bar graphs showing the number of five TSS clusters

1231 (middle) with different promoter types (right).

1232 (D) Line plots showing the average enrichments of BRG1 (EgfpKD and IpmkKD) and IgG at TSS with two

1233 promoter types (see the total on left, H3K4me3-Only and bivalent promoters are shown on top and bottom,

1234 respectively) and with five TSS clusters. Black, red, and gray lines indicate BRG1 intensity upon EgfpKD,

$1235 I p m k \mathrm{KD}$, and IgG intensity, respectively.

1236 (E) Bar graphs showing the average of differential ATAC-seq intensity ( $\log _{2} \mathrm{KD} / E g f p \mathrm{KD}$ ) upon IpmkKD 
1237 (blue) and $\operatorname{Brg} 1 \mathrm{KD}$ (green) at TSS with two promoter types.

1238 (F) Bar graphs showing the average of differential ATAC-seq intensity ( $\left.\log _{2} \mathrm{KD} / E g f p \mathrm{KD}\right)$ upon IpmkKD

1239 (blue) and $\operatorname{Brg} 1 \mathrm{KD}$ (green) at five TSS clusters with H3K4me3-Only (left) and bivalent promoters (right).

1240 (E and F) $P$-values were derived using Wilcoxon signed rank test $\left(* \mathrm{P}<0.01 ; * * \mathrm{P}<1 \times 10^{-4}\right.$; *** $\mathrm{P}<1 \times 10^{-}$

$1241{ }^{10} ;$ ns, not significant).

1242 (G) Examples of BRG1 (EgfpKD and IpmkKD), IPMK, IgG CUT\&RUN, and ATAC-seq (EgfpKD,

1243 IpmkKD, Control, and BrglKD) assays at TSS of Adpgk (left), Htatsfl (middle), and Zfp942/943 (right).

1244 Center and Up/Downstream regions are marked as purple and red/orange boxes on top, respectively. TSS

1245 are marked with red boxes (bottom) and dotted lines.

1246 (H) Examples of BRG1 (EgfpKD and IpmkKD), IPMK, IgG CUT\&RUN, and ATAC-seq (EgfpKD,

1247 IpmkKD, Control, and BrglKD) assays at TSS of Xkr4. The BRG1 CUT\&RUN peaks (EgfpKD cells) and

1248 ATAC-seq peaks are marked as blue and green boxes on top. Center and Up/Downstream regions are

1249 marked as purple and red/orange boxes on top, respectively. TSS is marked with a red box (bottom) and a

1250 dotted line.

1251

1252

1253

Figure 6. Alteration in BRG1/ATAC upon IpmkKD affects gene expression, and IPMK-SMARCB1

1254 regulates a common set of genes.

1255

(A) RT-qPCR analysis of Ipmk expression after siRNA treatment. Error bars denote the standard deviation obtained from four biological replicates. The expression levels were normalized with respect to that of $\beta$ actin. $P$-value was calculated using Student's t-test.

1258

(B) Examples of BRG1 (EgfpKD and IpmkKD), IPMK CUT\&RUN, and ATAC-seq (EgfpKD, IpmkKD,

1259 Control, and BrglKD) assays at TSS of Nmrall (left) and Phactr3 (right). The BRG1 CUT\&RUN peaks

1260 (EgfpKD cells) and ATAC-seq peaks are marked as blue and green boxes on top. TSS are marked with red boxes (bottom) and dotted lines. RT-qPCR analysis of Nmrall (left) and Phactr3 (right) expression after siRNA treatment. Error bars denote the standard deviation obtained from four biological replicates. The expression levels were normalized with respect to that of $\beta$-actin. $P$-value was calculated using Student's ttest.

1265

(C) Volcano plots showing the differentially expressed genes (DEGs) upon IpmkKD, identified based on mRNA-seq data. Red and blue dots indicate DEGs that were found to be significantly up- and down-

1267 (D) Bar graphs showing the average of differential BRG1 (red) and ATAC-seq (blue) intensity upon regulated, respectively ( $P$-value $\leq 0.05$ and fold change $\geq 1.5)$.

1269 IpmkKD at BRG1 CUT\&RUN peaks (for BRG1 intensity) and at ATAC-seq peaks (for ATAC intensity) that are closest (within $2 \mathrm{~kb}$ for BRG1 peaks and within 500bp for ATAC-seq peaks) to the TSS of downregulated DEGs. $P$-values were derived using Wilcoxon signed rank test $\left(* * \mathrm{P}<1 \times 10^{-5} ; * * * \mathrm{P}<1 \times 10^{-10}\right)$.

1272 Student's t-test.

1273 (F) Examples of BRG1 (EgfpKD and IpmkKD), IPMK CUT\&RUN, and ATAC-seq (EgfpKD, IpmkKD, 
1274 Control, and BrglKD) assays at TSS of Lrrc61 (left) and Arhgap44 (right). The BRG1 CUT\&RUN peaks

1275 (EgfpKD cells) and ATAC-seq peaks are marked as blue and green boxes on top. TSS are marked with red

1276 boxes (bottom) and dotted lines. mRNA-seq analysis of Lrrc61 (left) and Arhgap44 (right) expression after

1277 siRNA treatment. $P$-value was calculated using Student's t-test.

1278 (G) Volcano plots showing the differentially expressed genes (DEGs) upon Smarcb1KD, identified based

1279 on mRNA-seq data. Red and blue dots indicate DEGs that were found to be significantly up- and down-

1280 regulated, respectively ( $P$-value $\leq 0.05$ and fold change $\geq 1.5$ ).

1281 (H) Heatmaps representing differential gene expression $\left(\log _{2}(\mathrm{KD} / \mathrm{Eg} f \mathrm{f} \mathrm{KD})\right)$ of up-regulated (clusters1, 2 , 1282 and 3) and down-regulated (clusters 4, 5, and 6) DEGs upon IpmkKD. The heatmaps are classified into six 1283 clusters based on the differential gene expression of IpmkKD and SmarcblKD cells.

1284 (I) Pie chart showing the proportion of IpmkKD-induced DEGs that are regulated in the same manner (red, 1285 clusters 1 and 5) or the opposite manner (green, clusters 2 and 4) upon IpmkKD and Smarcb1KD.

1286 (J) Venn diagrams representing up-regulated DEGs (top) and down-regulated DEGs (bottom) in IpmkKD 1287 (orange) and SmarcblKD (blue) cells. The $P$-values indicate the significance of the overlap between the 1288 two groups.

1291 (A) A model displaying the physical interactions between IPMK and SMARCB1 (left). For these physical 1292 interactions, the exons 3, 4, and 6 of IPMK (orange boxes) and the Rpt1 and Rpt2 (particularly N-terminal $1293 \beta$ sheets) domains of SMARCB1 (red boxes) are required. An additional model showing our speculation 1294 on position of IPMK within the SWI/SNF complex, directly interacting with SMARCB1, BRG1, and 1295 BAF155 (right).

1296 (B) In WT mESC (left), IPMK regulates appropriate BRG1 localization (probably via physical interaction 1297 with various subunits of SWI/SNF complex) and chromatin accessibility at NFR (nucleosome free region) 1298 of TSS. Upon Ipmk depletion (right), BRG1 localization is perturbed, resulting in decreased chromatin 1299 accessibility at NFR of TSS. 
Figure 1-figure supplement 1. Various subunits of SWI/SNF complex and histones are IPMKproximal/interacting proteins.

1304 A volcano plot showing the relative abundance and significance $(P$-value) of biotinylated proteins related to SWI/SNF complex, histones, and two negative controls (right). A dotted line within the volcano plot indicates the $P$-value $=0.05$. The relative abundance (abundance ratio) was derived by comparing the fold enrichment of target proteins in IPMK-APEX2-expressed to APEX2-expressed HEK293 cells. $P$-value was calculated using Student's t-test.

Figure 2-figure supplement 1. IPMK physically binds to SMARCB1 and other subunits of the 1311 SWI/SNF complex.

1312 (A) IPMK and IgG were immunoprecipitated from NIH3T3 cells and subjected to immunoblotting.

1313 (B) IPMK and IgG were immunoprecipitated from NIH3T3 cells in the presence of dithiobis (succinimidyl 1314 propionate) (DSP), an established crosslinker, and subjected to immunoblotting.

1315 (C) SMARCB1 and IgG were immunoprecipitated from wild-type (WT) and IPMK-depleted (KO) MEF 1316 cells and subjected to immunoblotting.

1317 (D) E14Tg2a cells and NIH3T3 cells were non-transfected (NT) or transfected with siRNA against Egfp 1318 (EgfpKD), Ipmk (IpmkKD), and Smarcb1 (Smarcb1KD), and then subjected to immunoblotting.

1319 (E) E14Tg2a cells were transfected with siRNA against Egfp (EgfpKD) and Smarcb1 (Smarcb1KD), 1320 immunoprecipitated with IPMK and $\mathrm{IgG}$, and subjected to immunoblotting.

1321 (F) HEK293T cells were co-transfected with GST-IPMK and FLAG-SMARCB1 or FLAG (a control 1322 vector), followed by FLAG immunoprecipitation and immunoblotting.

1323 (G) HEK293T cells were transfected with GST-IPMK or GST (a control vector), followed by GST pull1324 down and immunoblotting.

1325 (H) Coomassie blue staining (left) and immunoblots of native SWI/SNF complex purified from FLAG1326 DPF2 HEK293T cell line.

1327 (I) Native SWI/SNF complex purified from FLAG-DPF2 HEK293T cell line and purified GST-IPMK or 1328 GST were co-incubated, followed by GST pull-down and immunoblotting (left). Coomassie blue staining of purified GST and GST-IPMK proteins (right).

Figure 3-figure supplement 1. Domain mapping of the interaction between IPMK and SMARCB1.

1332 (A) IPMK and SMARCB1 domain interaction test in yeast strain AH109, containing the HIS3 reporter gene. 1333 Yeast cells were co-transformed with either the GAL4-BD fusion plasmid pGBKT7 or pGBKT7-IPMK and 1334 the GAL4-AD fusion plasmid pGADT7 with SMARCB1 deletion constructs or pACT2-SMARCB1. IPMK 1335 interacts with 99-245 or 99-319 SMARCB1 deletion constructs, whereas IPMK does not interact with 99185 SMARCB1 constructs. The yeast cells were spread on the selection medium lacking leucine and tryptophan (SD-LW) to select co-transformants of bait and prey vectors. Specific interactions between bait and prey proteins were monitored by cell growth on a selection medium lacking leucine, tryptophan, and histidine (SD-LWH). 3-AT (3-amino-1,2,4-triazole) was used to suppress leaky HIS3 expression in 
transformants to obtain an accurate phenotype. A schematic diagram of the SMARCB1 domain map with the number of amino acid sequences is presented on the right. The IPMK-binding sites (Rpt1 and Rpt2) are highlighted in red.

1343 (B, C, and D) HEK293T cells were co-transfected with FLAG-IPMK and GST (a control vector) or GSTSMARCB1 Rpt1 fragments (B and D) and GST-MARCB1 Rpt2 fragments (C and D), followed by immunoprecipitation with FLAG antibody and subjected to immunoblotting. The specific IPMK-binding Rpt1 (B) and Rpt2 (C) domains are highlighted in red. Arrows and cylinders indicate $\beta$ sheets and $\alpha$ helices, respectively.

(E) HEK293T cells were co-transfected with FLAG-IPMK and GST (a control vector), GST-SMARCB1, or GST-SMARCB1 without Rpt1 and Rpt2 (GST- $\Delta 186-371$ ), followed by immunoprecipitation with FLAG antibody, and subjected to immunoblotting.

(F) Schematic diagram of human SMARCB1 and SMARCB1 without Rpt1 and Rpt2 ( $1866-371)$. The IPMK-binding sites (Rpt1 and Rpt2) are highlighted in red with the number of amino acid sequences.

\section{Figure 4-figure supplement 1. Chromatin fraction assay}

1355

(A) E14Tg2a cells were fractionated into the cytoplasm, nucleoplasm, and chromatin fractions. Immunoblotting with IPMK, SMARCB1, and fractionation markers was then performed. (B) NIH3T3 cells were fractionated into the cytoplasm, nucleoplasm, and chromatin fractions. Immunoblotting with IPMK, SMARCB1, and fractionation markers was then performed. (C) NIH3T3 cells were transfected with siRNA against Egfp (EgfpKD) and Smarcb1 (SmarcblKD) and then fractionated into the cytoplasm, nucleoplasm, and chromatin fractions. Immunoblotting with IPMK, SMARCB1, BRG1, and fractionation markers was then performed.

Figure 5-figure supplement 1. IPMK plays an important role in the maintenance of chromatin accessibility at promoter-TSS by regulating the BRG1 localization.

(A) Line plots showing the average enrichments of BRG1 ( $E g f p$ KD cells) at TSS with six clusters.

(B) A diagram displaying six clusters of TSS classified by the relative positions of BRG1 CUT\&RUN peaks (EgfpKD cells) respective to TSS and up/downstream regions of TSS (left). Bar graphs showing the number of six TSS clusters (right).

(C) Line plots showing the average enrichments of BRG1 (EgfpKD cells) at TSS with three clusters. ClusterC (red) contains cluster1-3, ClusterL (blue) contains cluster4-5, and ClusterR (green) resembles cluster6. peaks ( $E g f p \mathrm{KD}$ cells) respective to TSS and up/downstream regions of TSS (left). Bar graphs showing the number of three TSS clusters (right).

(E) Bar graphs showing the percentage of three TSS clusters exhibiting decreased (left) or increased (right) BRG1 intensity upon IpmkKD.

(F) Bar graphs showing the percentage of three TSS clusters (TSS exhibiting decreased BRG1 intensity 
upon IpmkKD) with $\mathrm{H} 3 \mathrm{~K} 4 \mathrm{me} 3-\mathrm{Only}$ (left) and bivalent (right) promoters.

(G) Box plots showing the BRG1 intensity at TSS with two promoter types (top: H3K4me3-Only promoters; bottom: bivalent promoters) and with three TSS clusters in EgfpKD (grey) and IpmkKD (red) cells. $P_{-}$ values were derived using Wilcoxon signed rank test $\left(* \mathrm{P}<1 \times 10^{-25} ; * * \mathrm{P}<1 \times 10^{-50} ; * * * \mathrm{P}<1 \times 10^{-100}\right)$.

(H) Bar graphs showing the average of differential ATAC-seq intensity ( $\left.\log _{2} \mathrm{KD} / E g f p \mathrm{KD}\right)$ upon IpmkKD (blue) and BrglKD (green) at three TSS clusters with H3K4me3-Only (left) and bivalent promoters (right). (I) Bar graphs showing the average of differential mRNA expression ( $\left.\log _{2} I p m k \mathrm{KD} / E g f p \mathrm{KD}\right)$ upon IpmkKD at TSS (TSS exhibiting decreased BRG1 intensity upon IpmkKD) with two promoter types. (H and I) $P$-values were derived using Wilcoxon signed rank test $\left({ }^{*} \mathrm{P}<0.01\right.$; ** $<1 \times 10^{-4}$; *** $\mathrm{P}<1 \times 10^{-10}$; ns, not significant).

Figure 6-figure supplement 1. mRNA transcriptome indicates that IPMK and SMARCB1 regulate a common set of genes.

(A) Bar graphs (left: $P$-value, right: $Q$-value) showing the gene ontology terms (biological process) of the

Cluster1 (top) and Cluster5 (bottom) DEGs that satisfied the threshold ( $P$-value $\leq 0.005$ ). Organ. denotes organization, and the dotted lines denote $P$-value $\leq 0.005$ (left) and $Q$-value $\leq 0.01$ (right).

(B) Volcano plots showing the differentially expressed genes (DEGs) upon IpmkKD (left) and SmarcblKD (right) in NIH3T3 cells, identified based on mRNA-seq data. Red and blue dots indicate DEGs that were found to be significantly up- and down-regulated, respectively ( $P$-value $\leq 0.05$ and fold change $\geq 1.5$ ). (C) Heatmaps representing differential gene expression $\left(\log _{2}(\mathrm{KD} / \mathrm{Eg} f p \mathrm{KD})\right)$ of up-regulated (clusters1, 2 , and 3) and down-regulated (clusters 4, 5, and 6) DEGs upon IpmkKD. The heatmaps are classified into six clusters based on the differential gene expression of IpmkKD and Smarcb1KD cells. (D) Pie chart showing the proportion of IpmkKD-induced DEGs that are regulated in the same manner (red, clusters 1 and 5) or the opposite manner (green, clusters 2 and 4) upon IpmkKD and SmarcblKD. (E) Venn diagrams representing up-regulated DEGs (top) and down-regulated DEGs (bottom) in IpmkKD (orange) and Smarcb1KD (blue) cells. The $P$-values indicate the significance of the overlap between the two groups.

Figure 7-figure supplement 1. Proposed model depicting the physical interaction of IPMK and

1408 An additional model showing our speculation on position of IPMK within the SWI/SNF complex in the presence of nucleosomes. 
1412 Supplementary Table 1. Yeast two-hybrid screening assay using IPMK as bait

1413

1414 Supplementary Table 2. Enriched protein complex-based sets from APEX2-mediated proximity

1415 labeling

1416

1417 Supplementary Table 3. Clustering analysis of DEG in mouse embryonic stem cells

1418

1419 Supplementary Table 4. Clustering analysis of DEG in mouse fibroblasts

1420

1421 
A

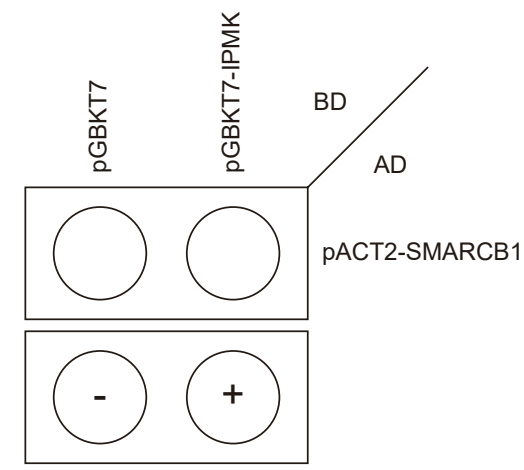

- : negative control

+ : positive control

B

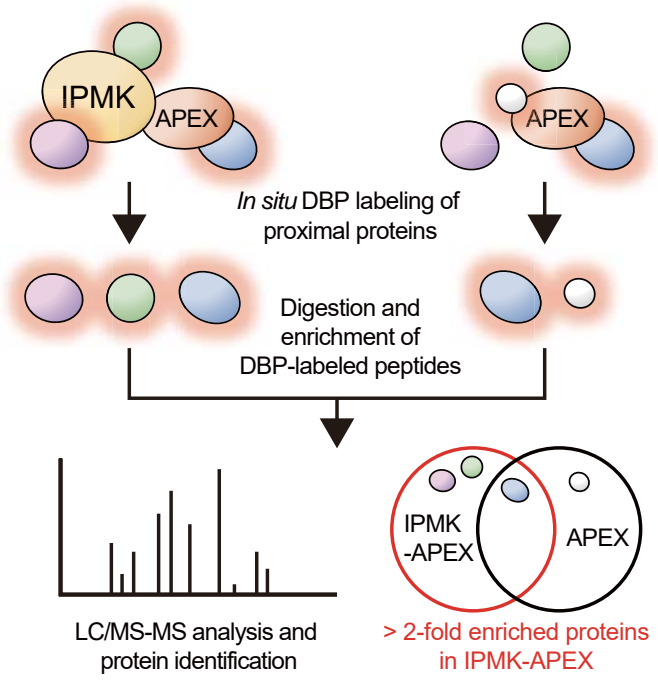

ADE2 expression

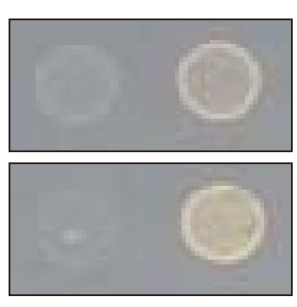

SD-LWA
HIS3 expression

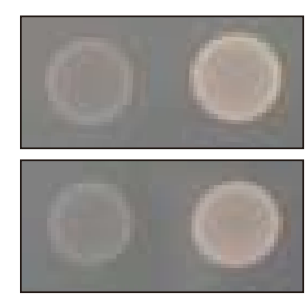

SD-LWH(+5mM 3-AT)

C

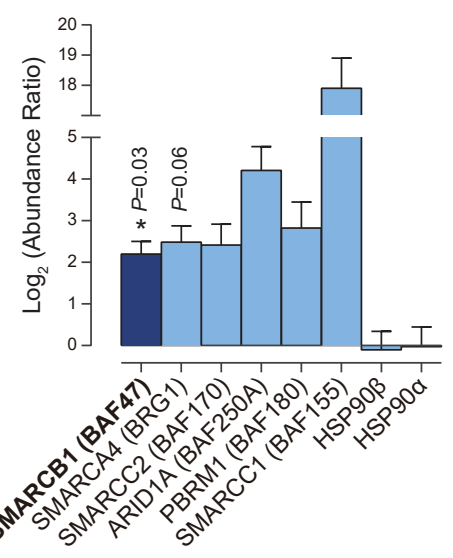

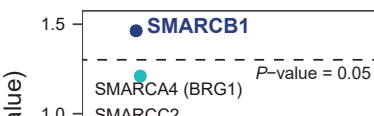

$$
\begin{aligned}
& \text { IN } 1.0 \text { - SMARCC2 } \\
& \text { PBRM1 } 1 \\
& \text { 1 } \\
& \text { HSP9Oß } \\
& 0.0-8 \text { HSP90a } \\
& \begin{array}{lllllllllll}
\hline & 2 & 4 & 6 & 8 & 10 & 12 & 14 & 16 & 18
\end{array} \\
& \log _{2} \text { (Abundance Ratio) }
\end{aligned}
$$


A

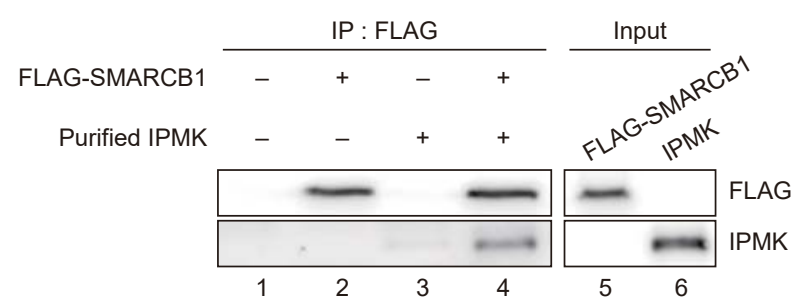

B

\begin{tabular}{|c|c|c|c|c|c|}
\hline & & Input & & & \\
\hline SMARCE & - & + & + & - & \\
\hline AG_IPM & - & + & + & - & \\
\hline
\end{tabular}

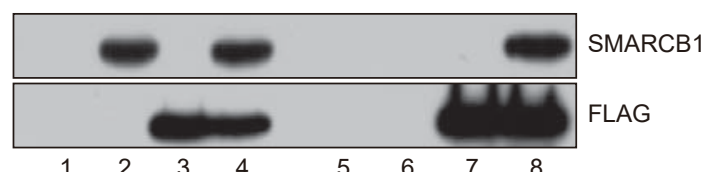

FLAG-IPMK

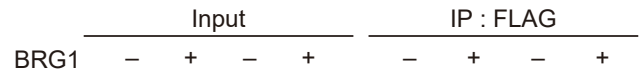

FLAG-IPNK
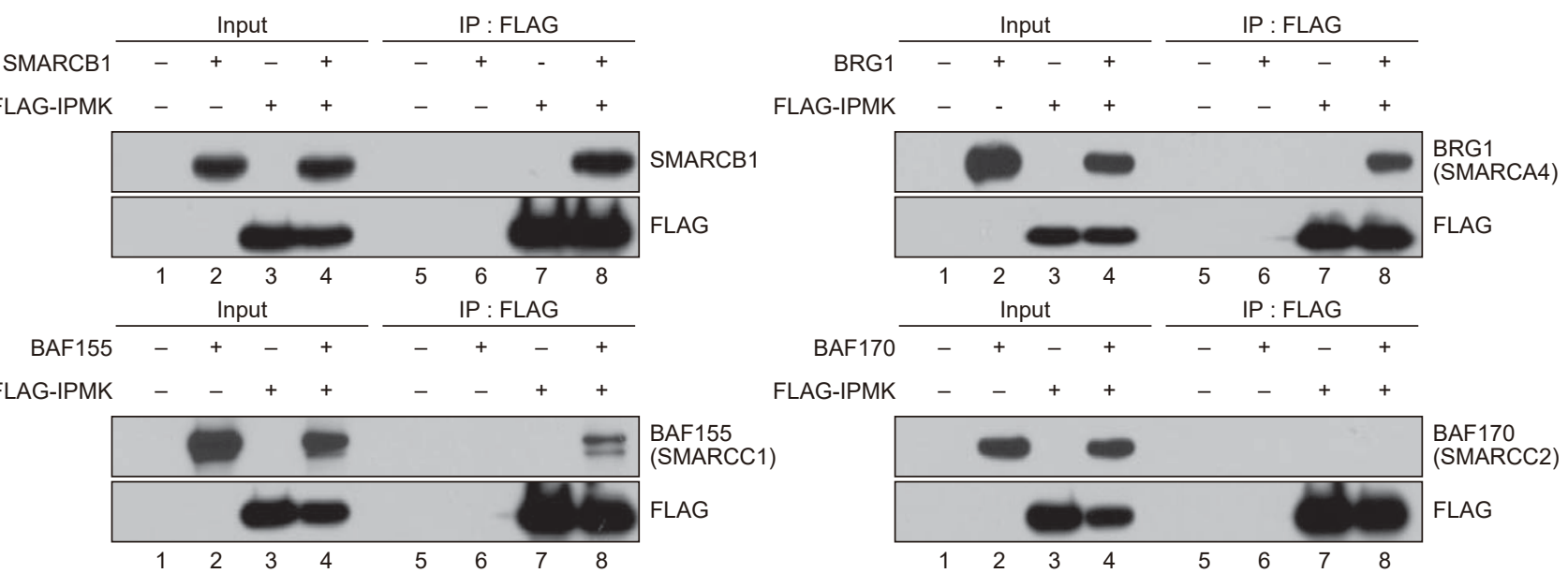

C

E
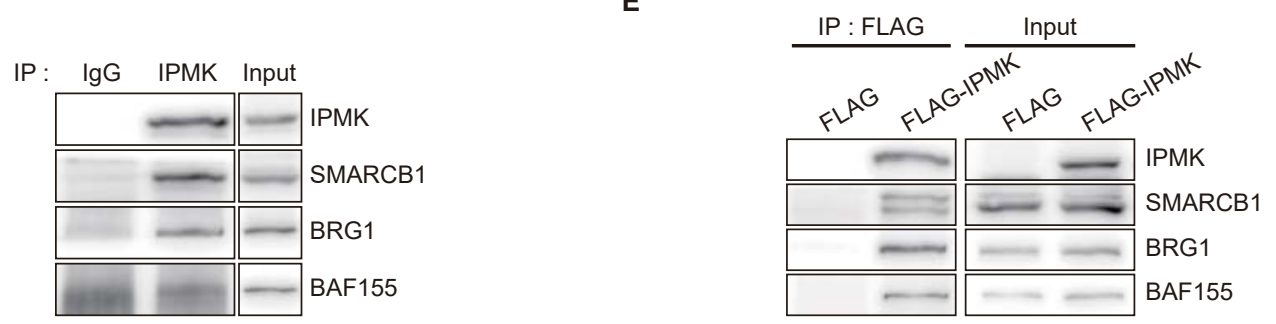

IP : IgG SMARCB1 Input
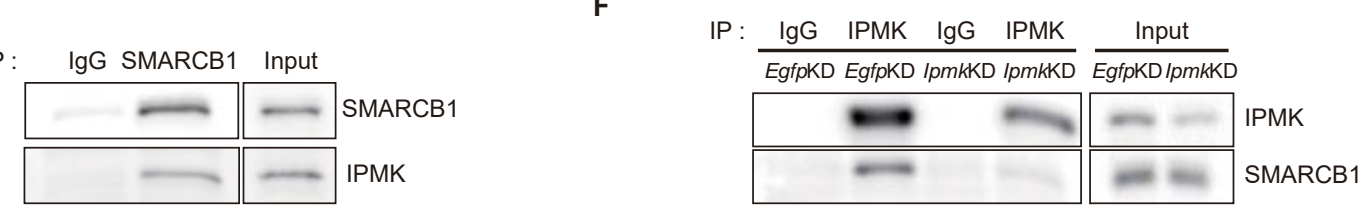
A

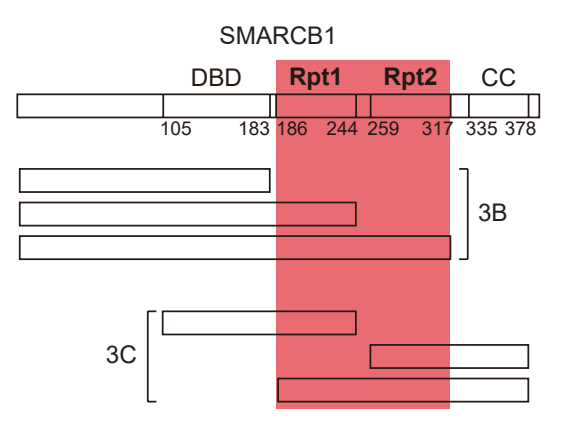

B

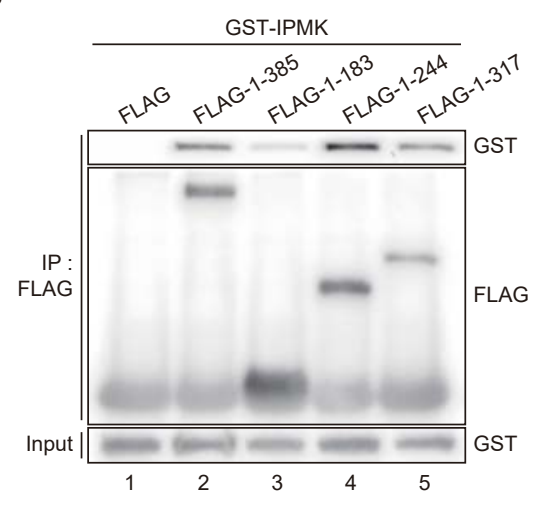

C

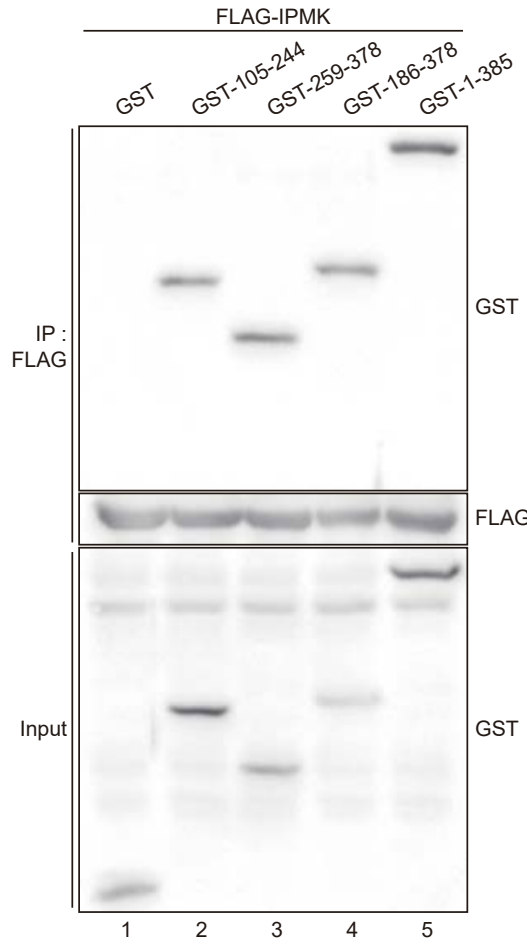

D

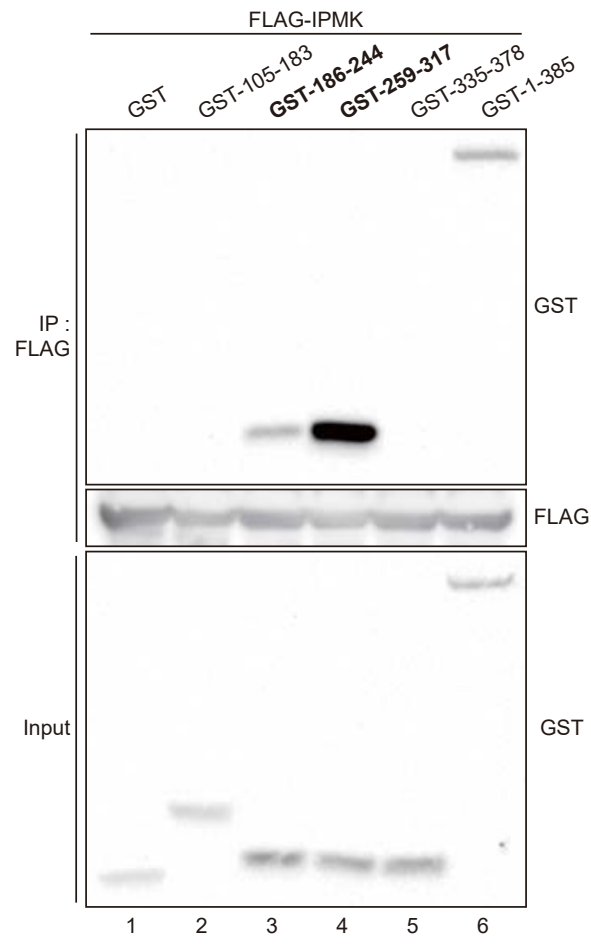

E

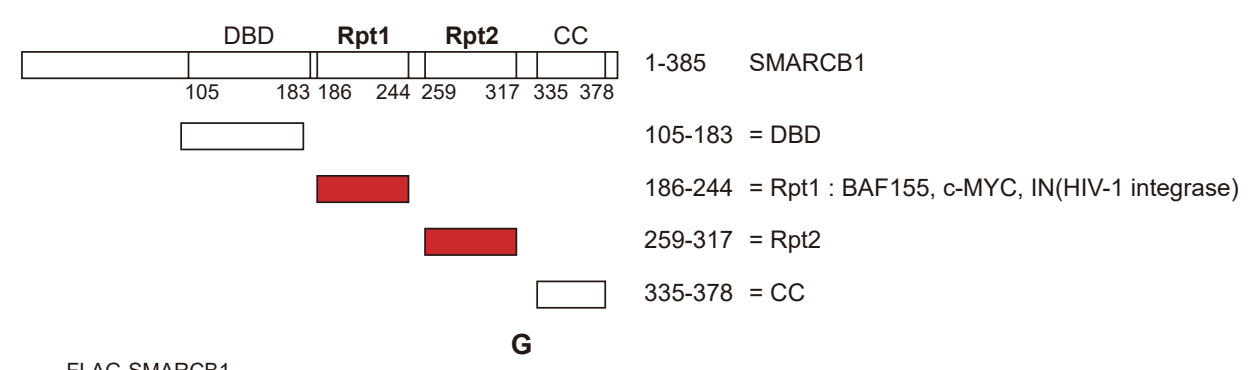

F

FLAG-SMARCB1

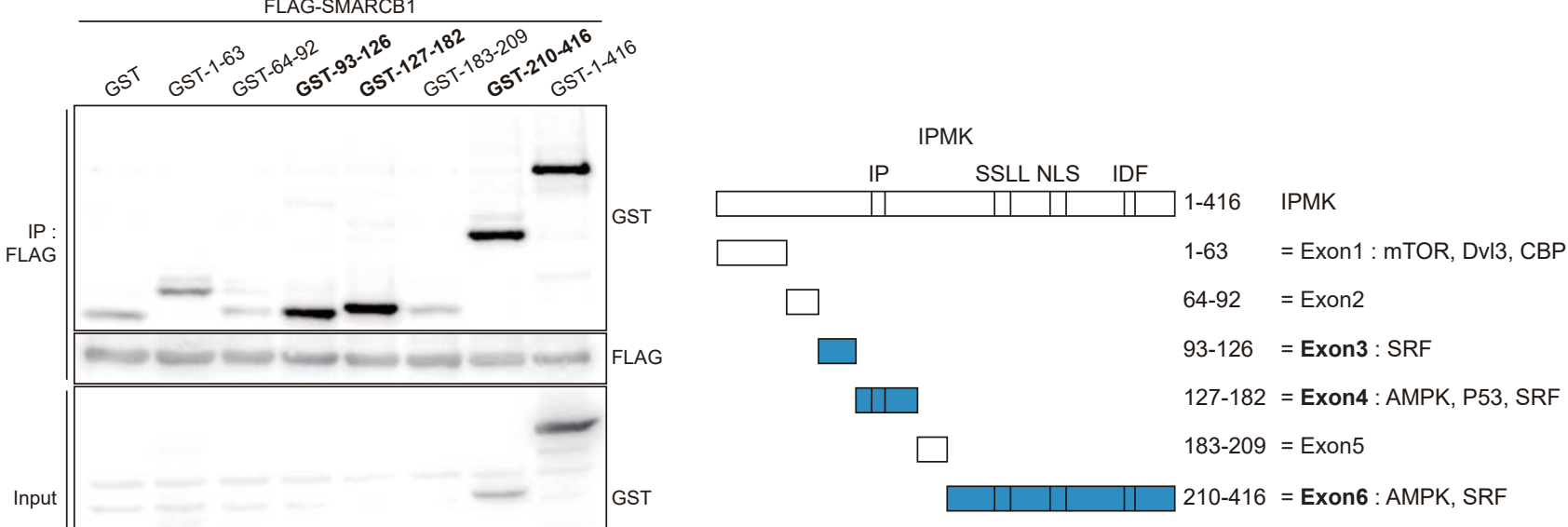




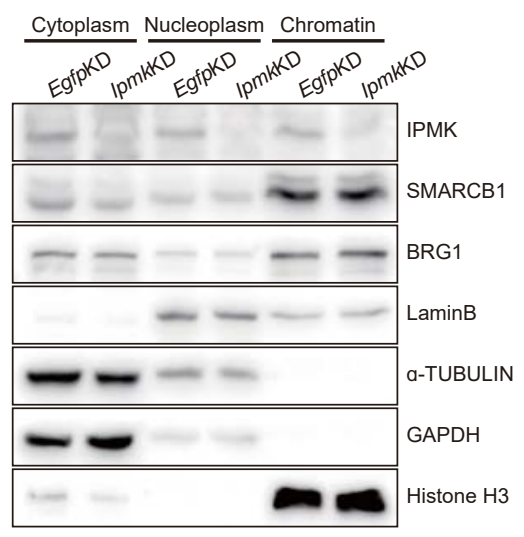

B
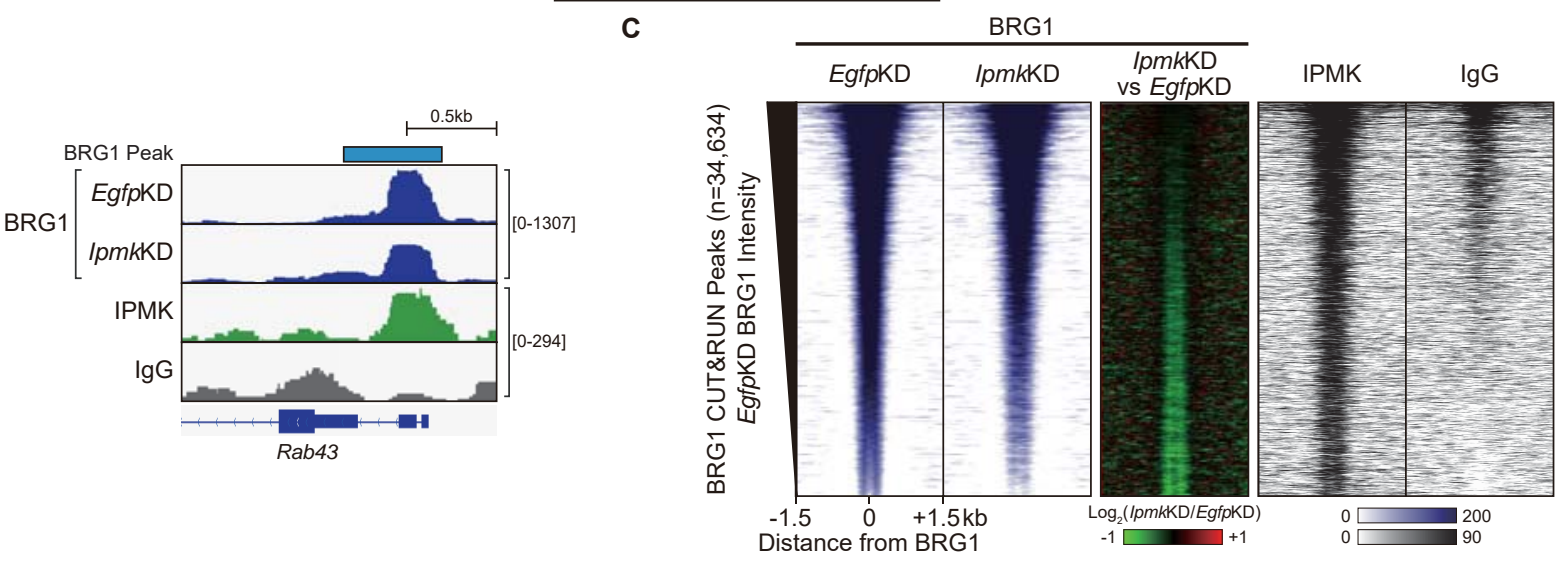

D

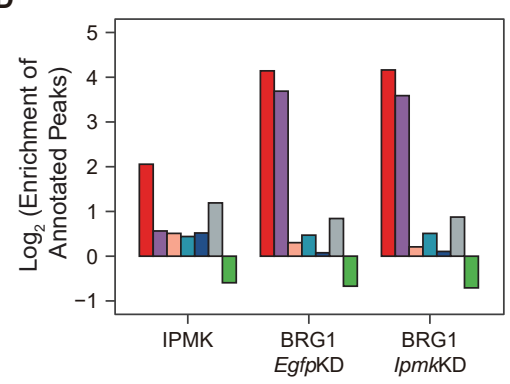

E

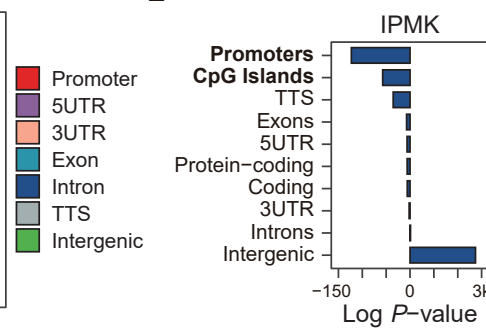

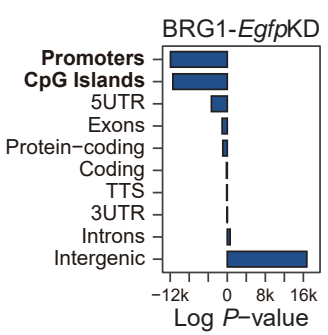

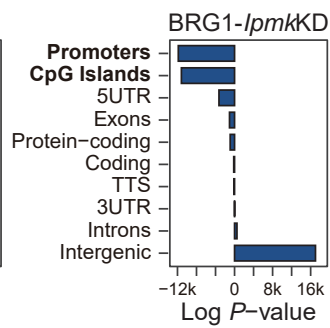

F

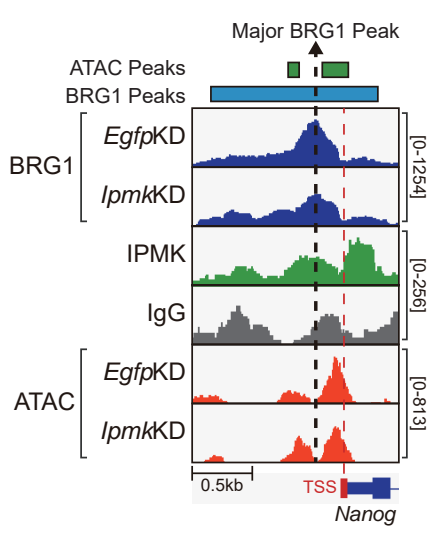

H

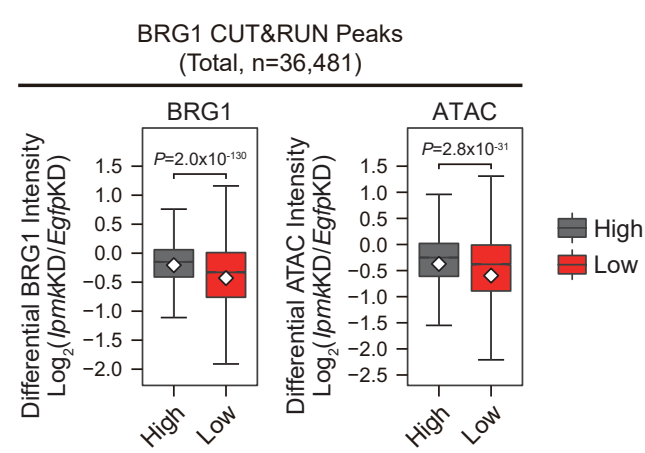

G

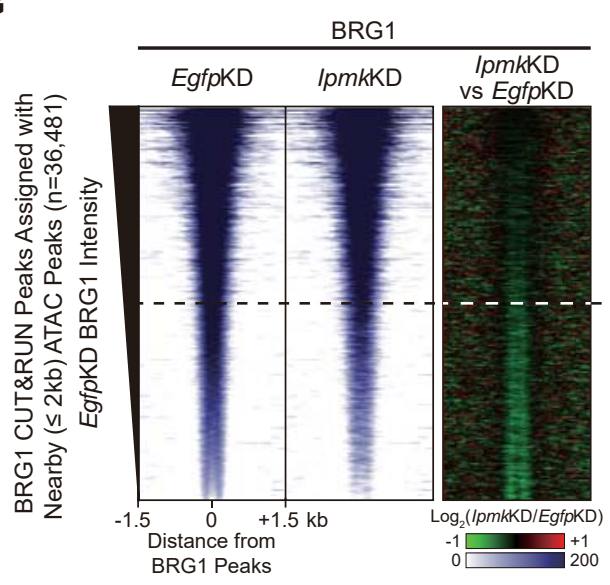

I

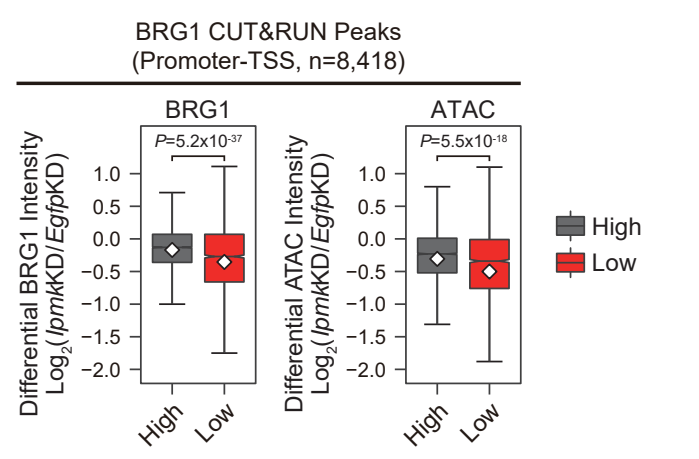


A

Center $[-200+100 \mathrm{bp}]$

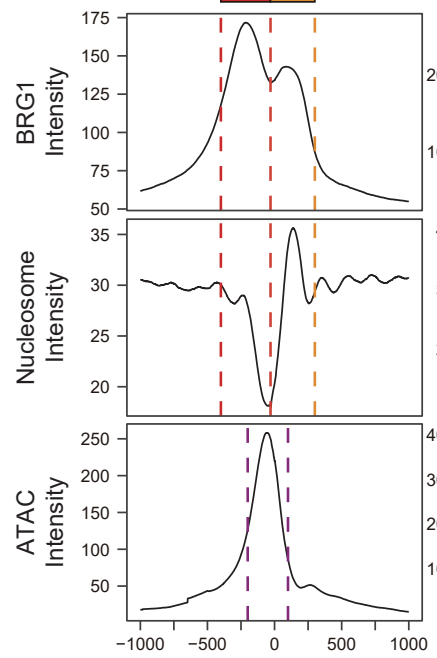

Distance from TSS (bp)
Upstream [-400 -30 bp] \_ Downstream [-30 +300 bp]

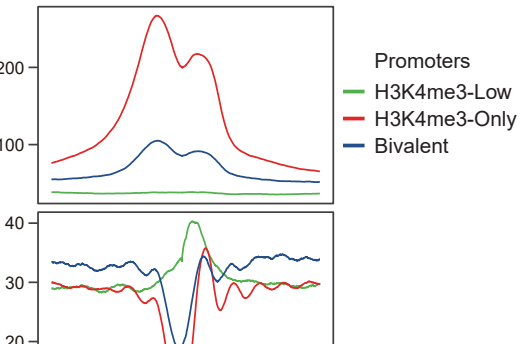

$\circ$
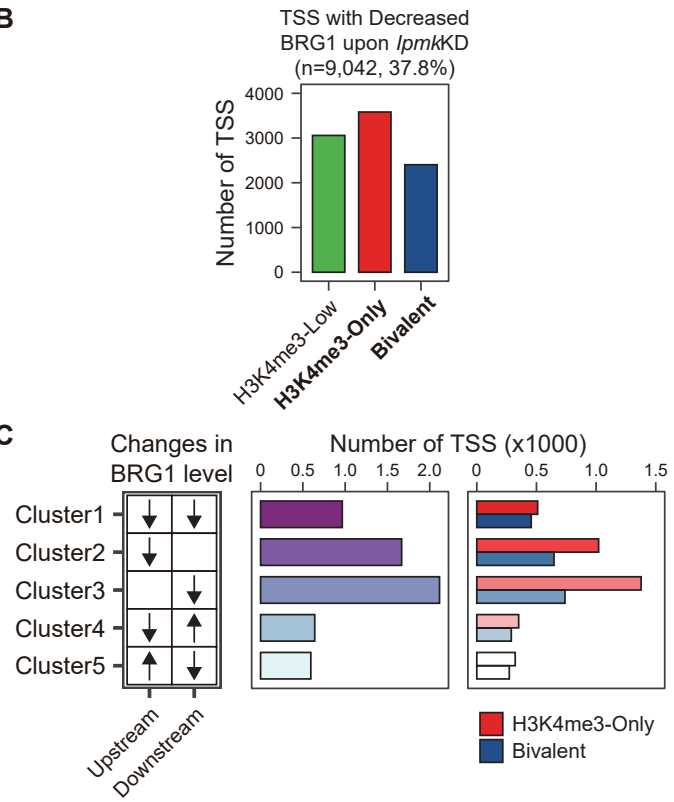

D

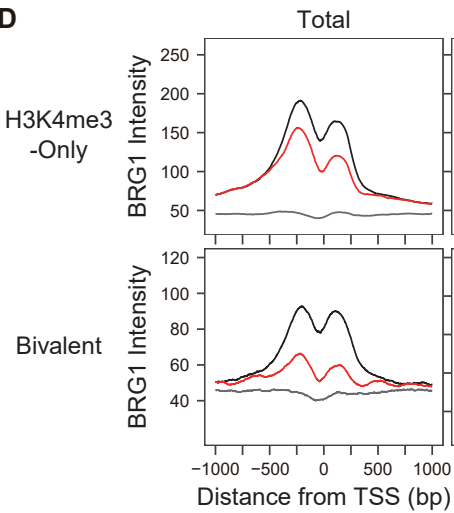

E
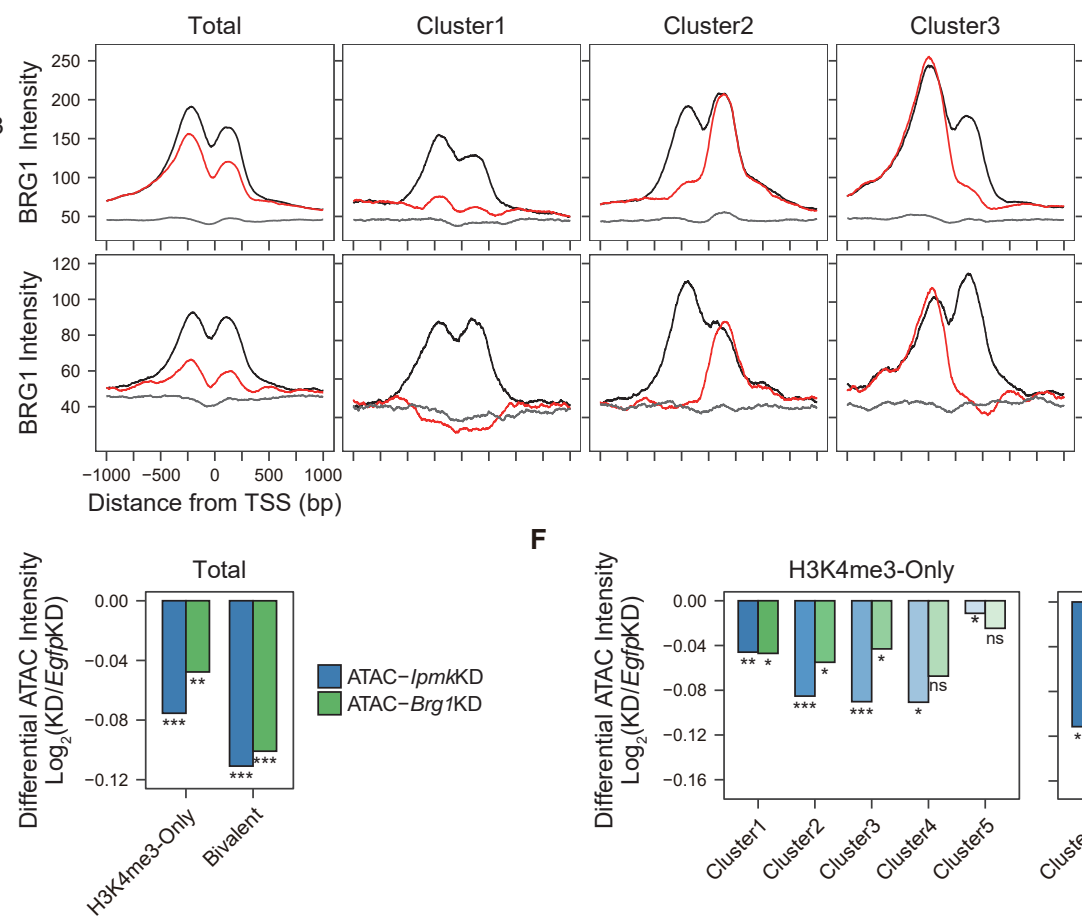

Cluster4

Cluster5

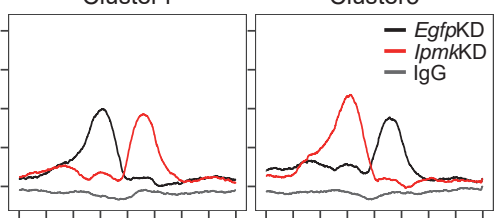

G
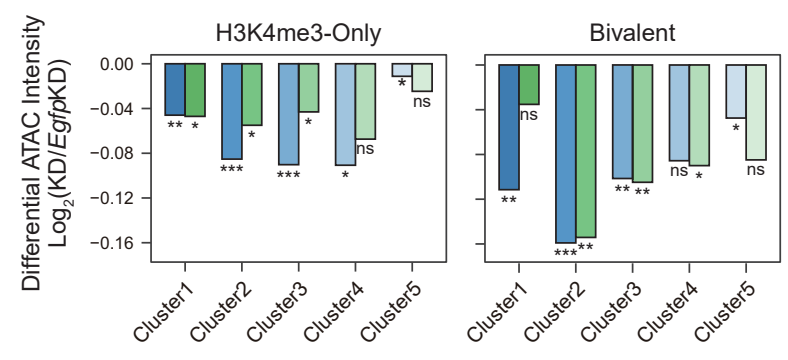

ATAC-IpmkKD

ATAC-Brg1KD

H
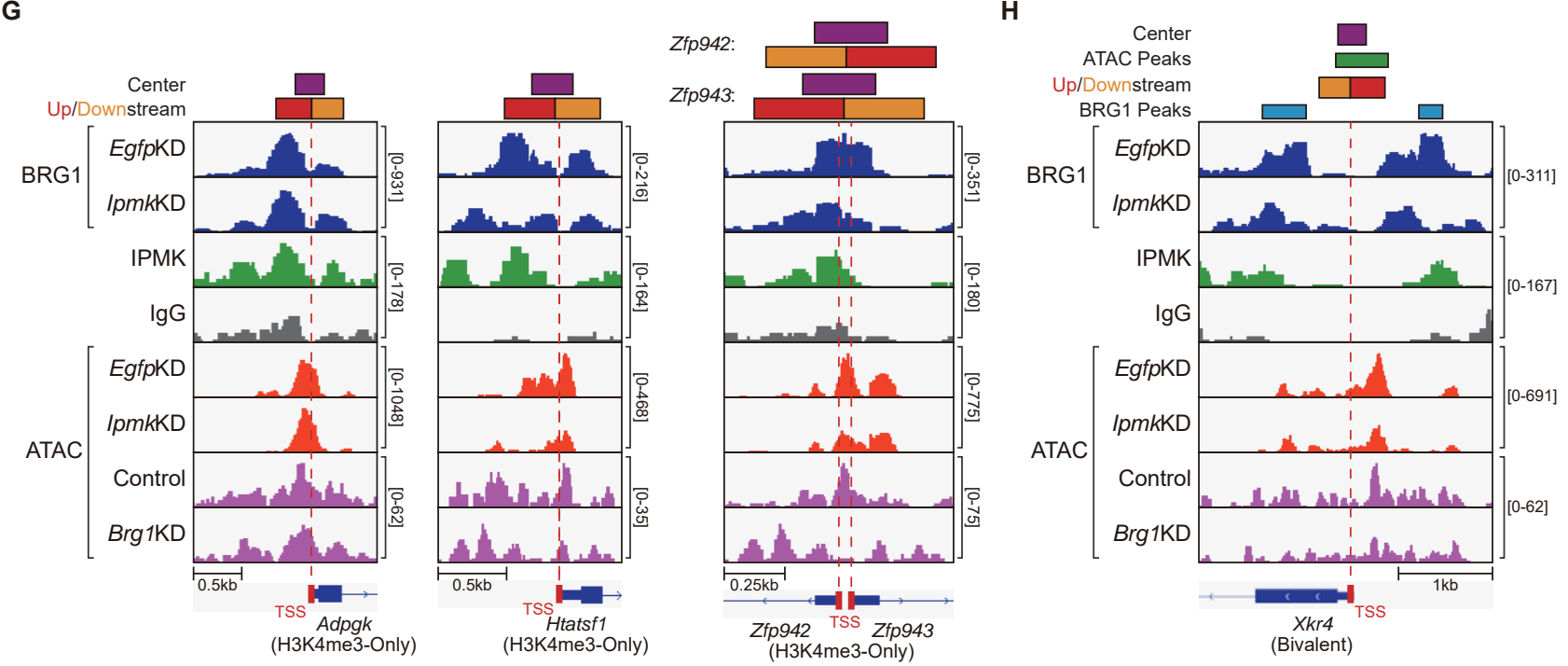
A

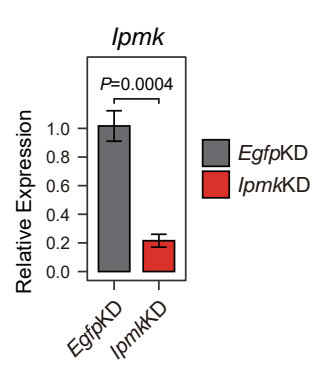

C

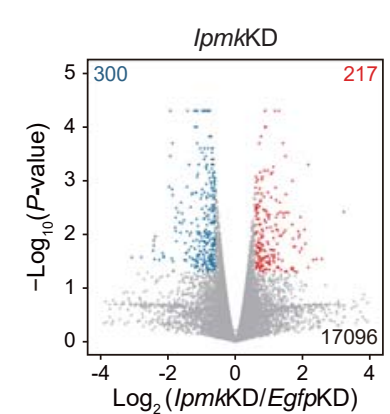

B

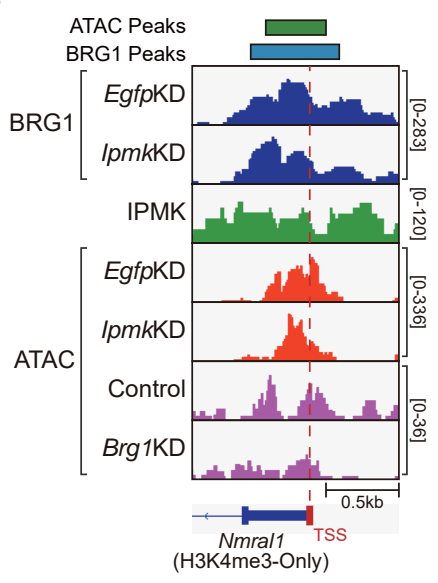

G

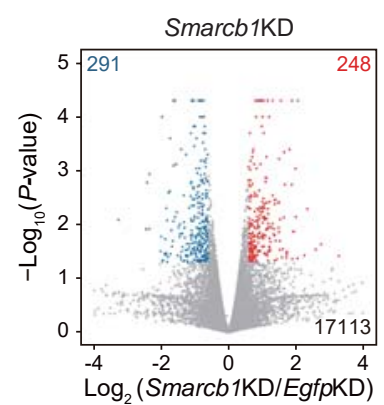

$\square$ Down-regulated $\square$ Not DEGs $\square$ Up-regulated

D

Down-regulated DEG

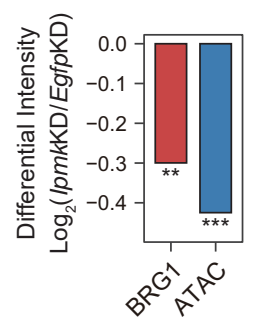

BRG1-IpmkKD

ATAC-IpmkKD

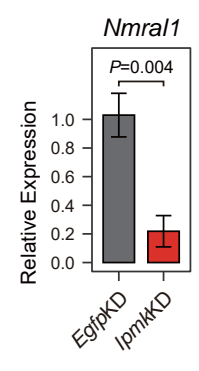

H

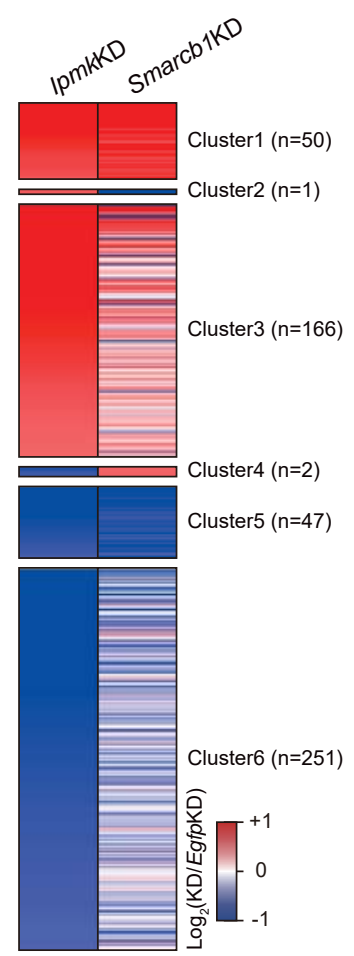

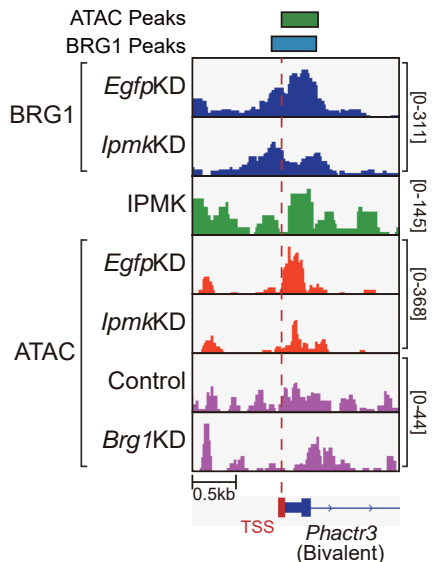

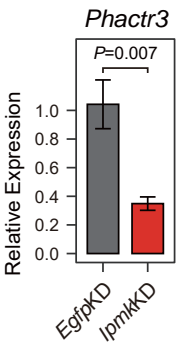

E

$\mathbf{F}$
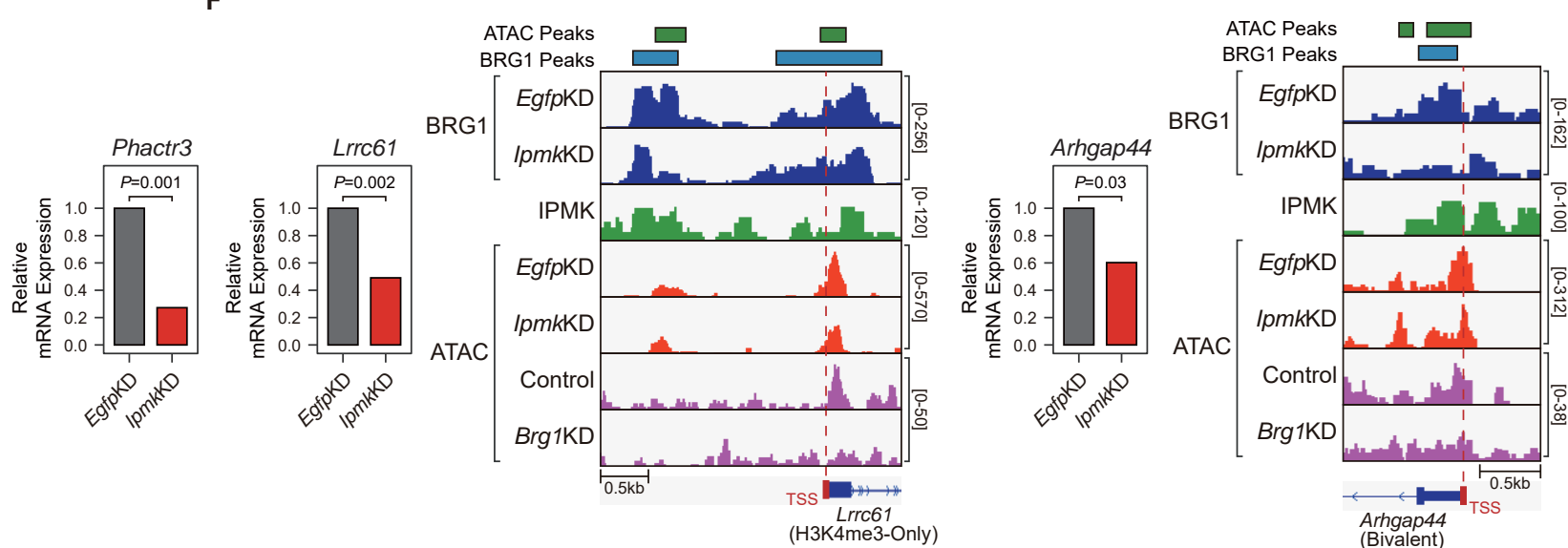

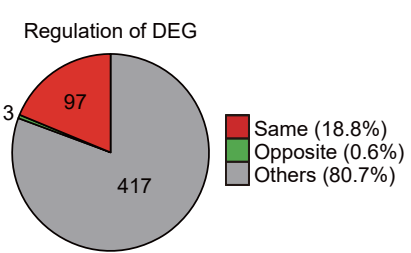

J

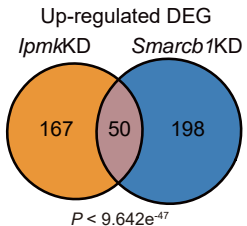

Down-regulated DEG

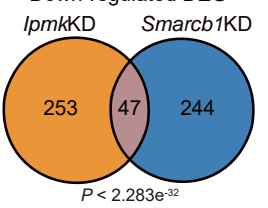

(Bivalent) 
A

Physical Interaction
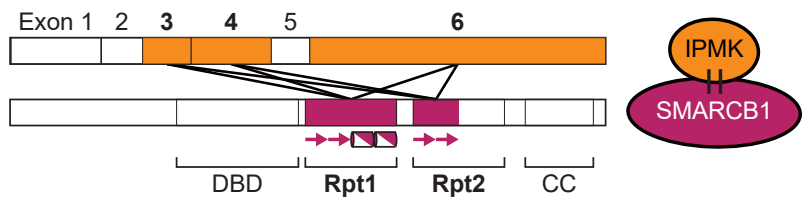

B

\section{WT mESC}

Appropriate BRG1 Localization Normal Chromatin Accessibility at TSS

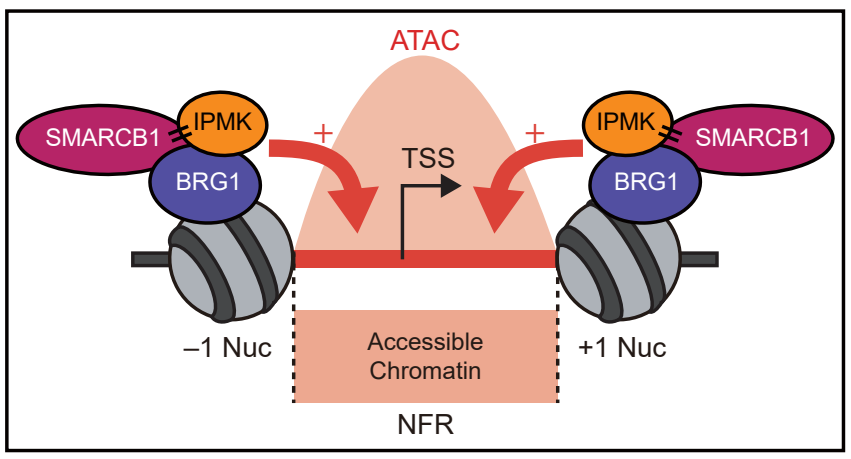

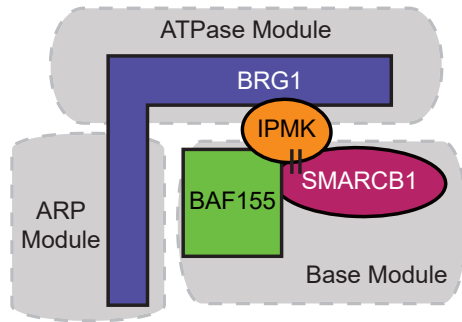

\section{Ipmk Depletion}

Perturbed BRG1 Localization

Decreased Chromatin Accessibility at TSS

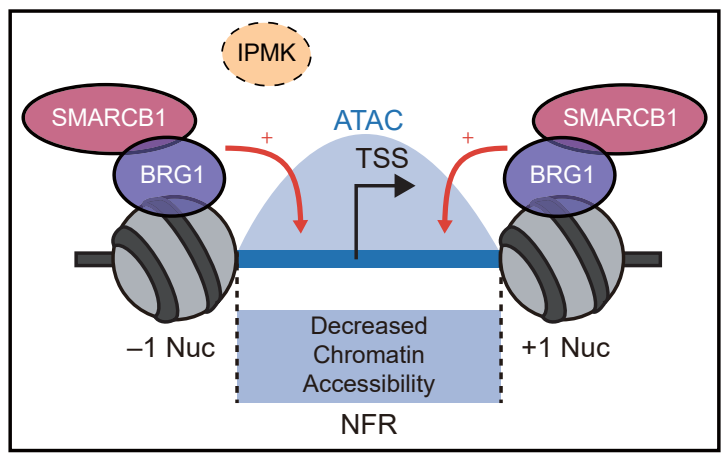




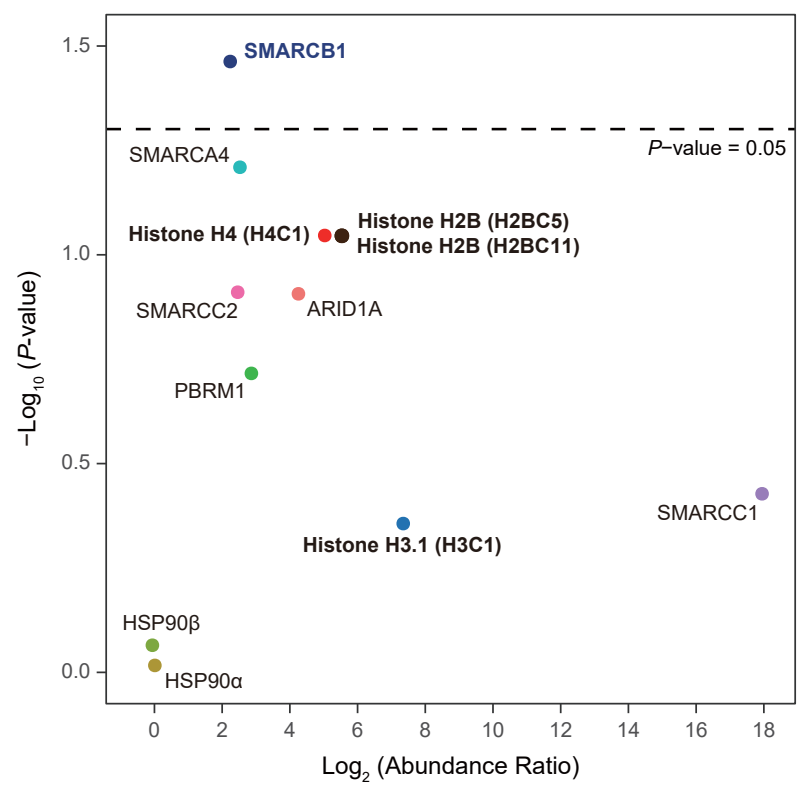


A

\begin{tabular}{|c|c|c|c|}
\hline \multicolumn{2}{|c|}{ IP } & \multirow[b]{2}{*}{ Input } & \\
\hline $\lg G$ & IPMK & & \\
\hline & $=$ & 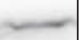 & IPMK \\
\hline & $=$ & - & SMARCB1 \\
\hline & 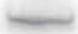 & - & BRG1 \\
\hline
\end{tabular}

B

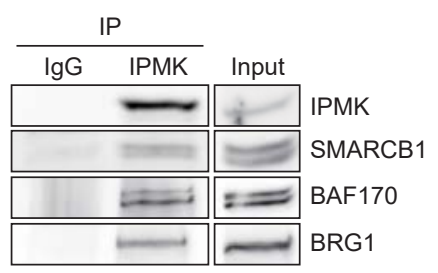

C

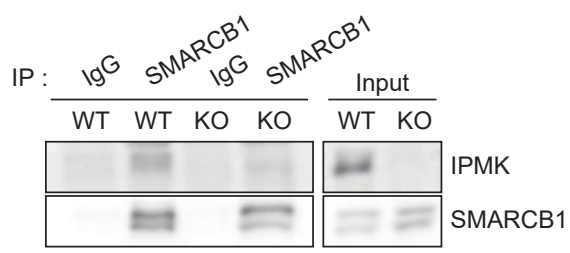

D

Mouse embryonic stem cells
E14Tg2a

\begin{tabular}{|c|c|c|c|c|}
\hline & & & & \\
\hline$=$ & - & - & 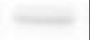 & SMARCB1 \\
\hline$=$ & - & - & - & BAF155 \\
\hline$x$ & $=$ & $=$ & $=$ & BAF170 \\
\hline$=$ & $=$ & $=$ & 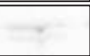 & BAF250A \\
\hline$=$ & $=$ & $=$ & & a-TUBULIN \\
\hline
\end{tabular}

E

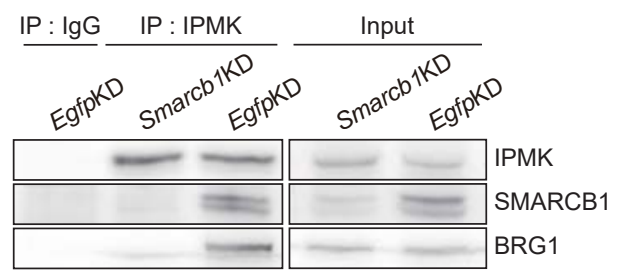

$\mathbf{F}$

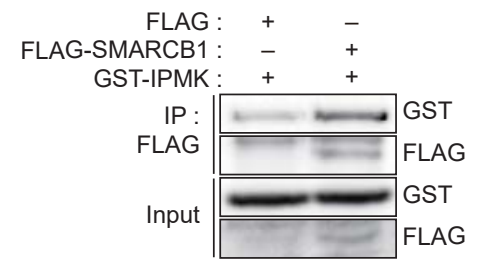

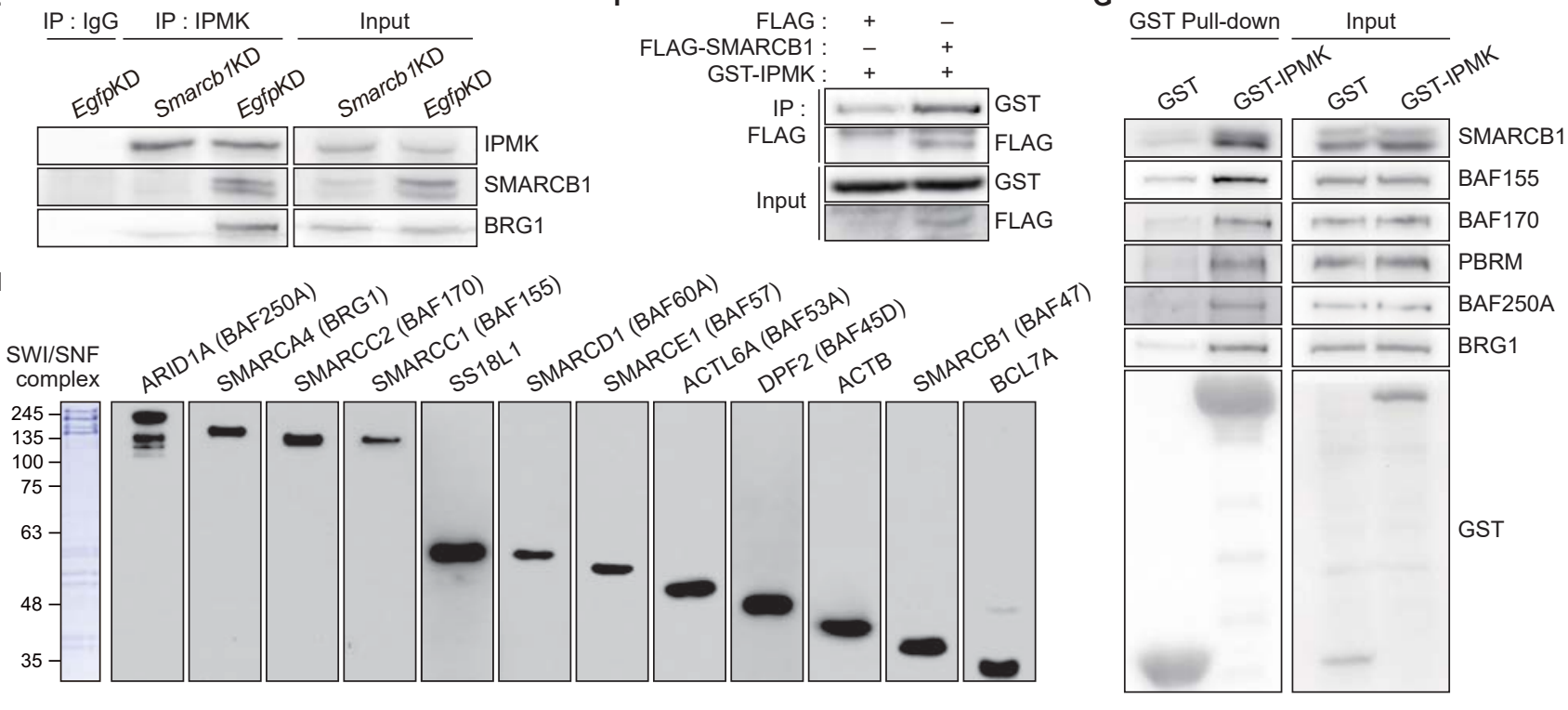

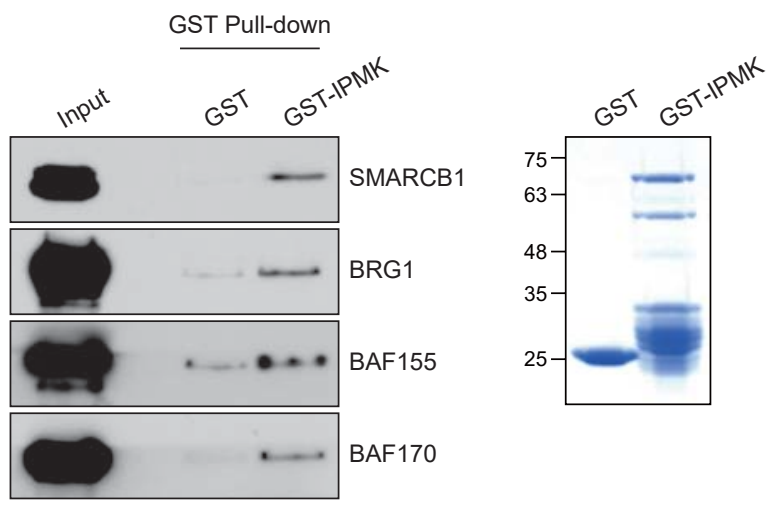

Mouse embryonic fibroblasts NIH3T3

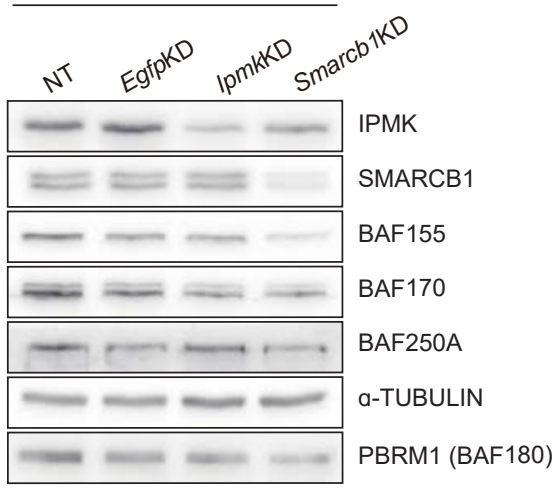

G 
A

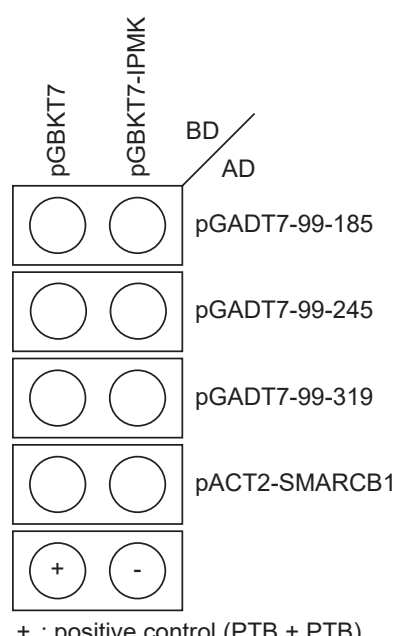

+ : positive control (PTB + PTB)

- : negative control

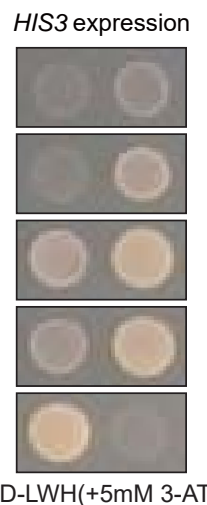

B

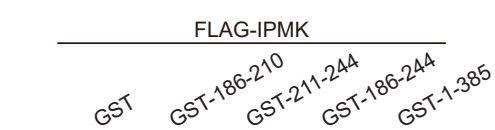

C
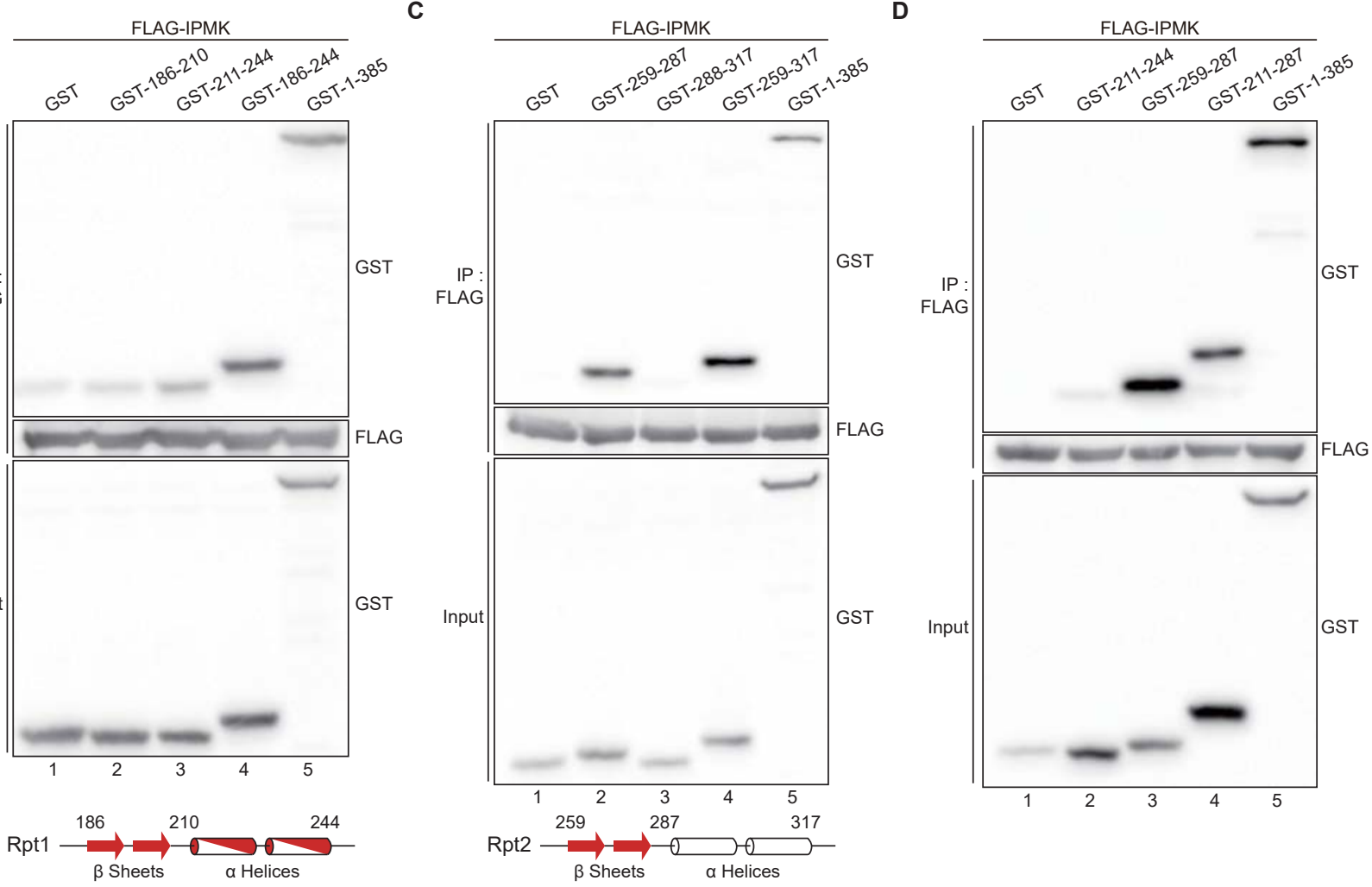

FLAG

E

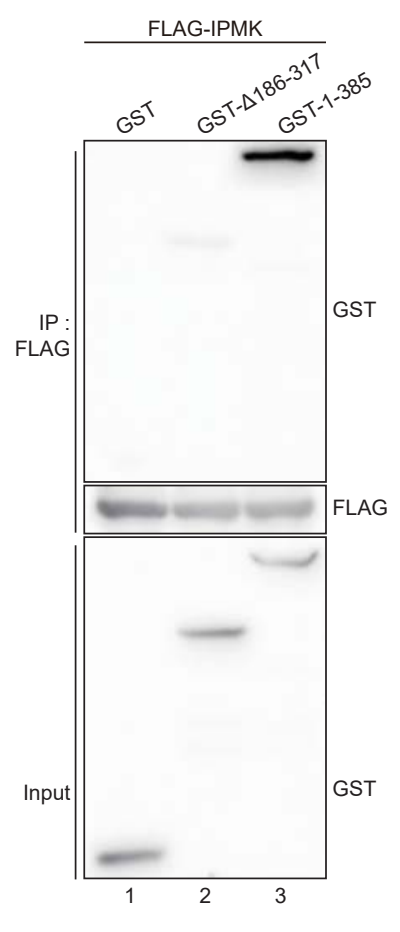

SMARCB1

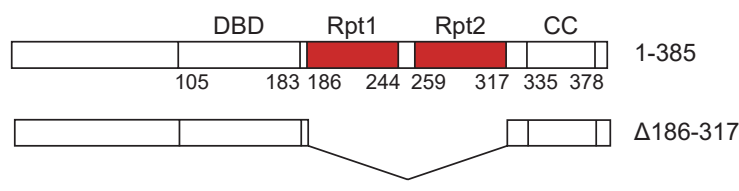


A Mouse embryonic stem cells

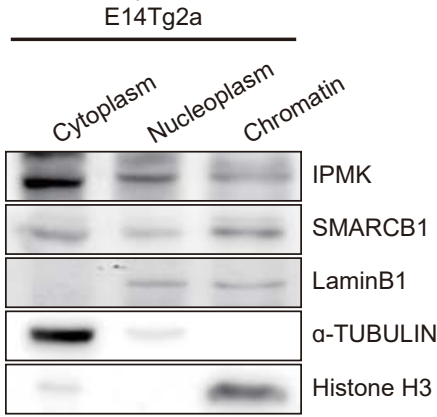

B Mouse embryonic fibroblasts $\mathrm{NIH} 3 \mathrm{~T} 3$

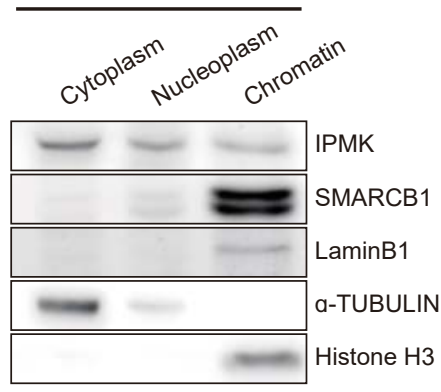

C

Cytoplasm Nucleoplasm Chromatin

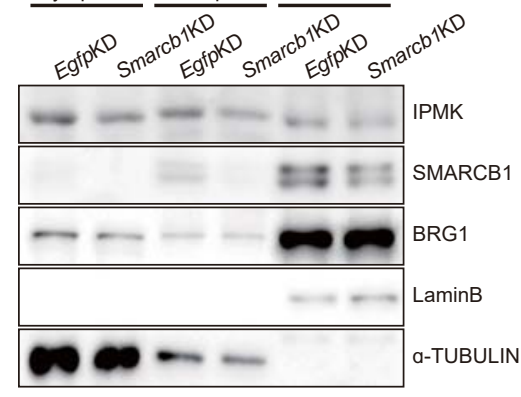


A
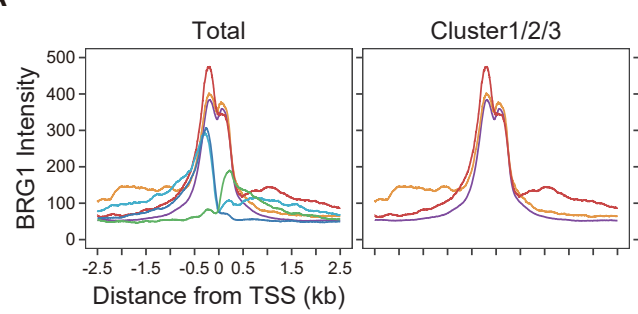

c

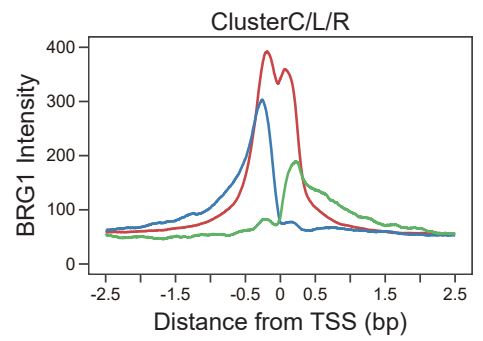

D

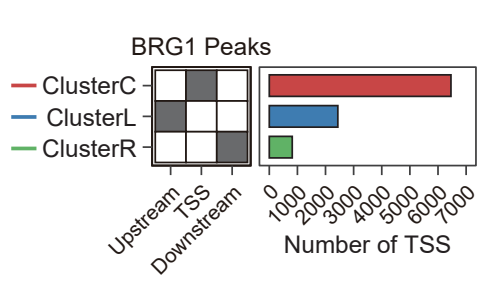

E

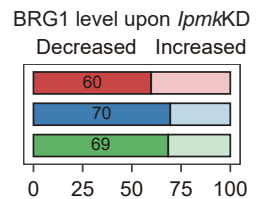

Percentage of TSS (\%)
BRG1 Peaks

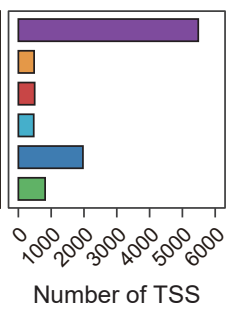

F

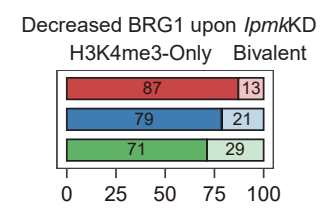

Percentage of Promoters (\%)
G

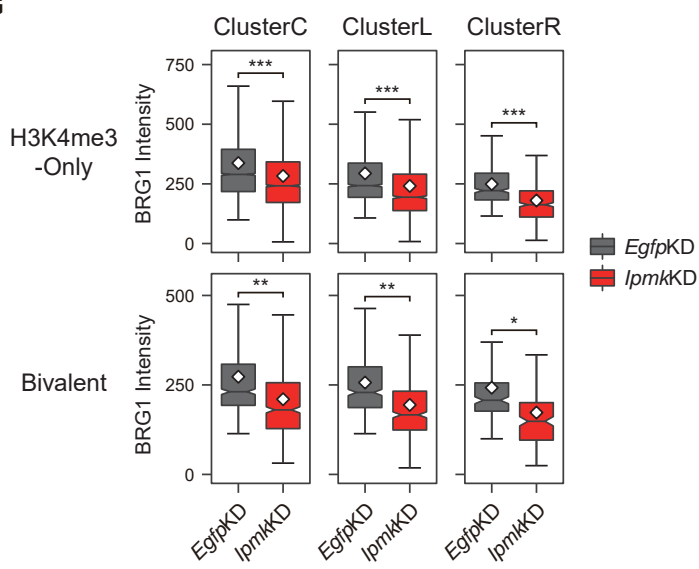

H

I

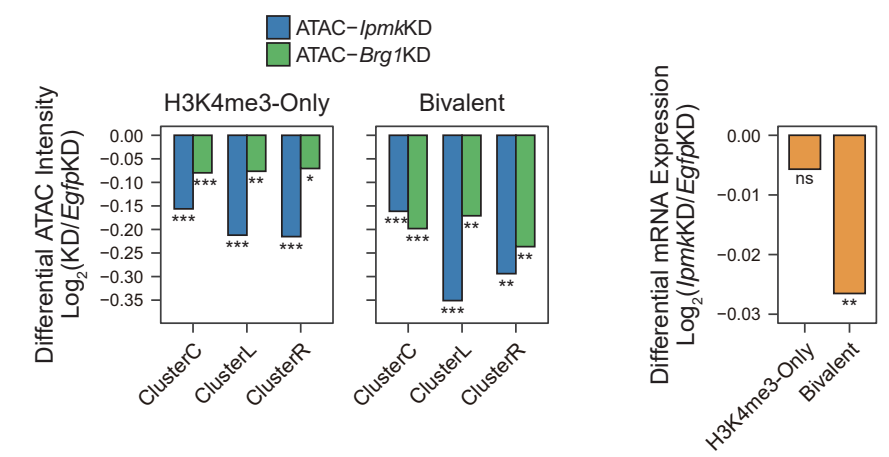


protein-containing complex subunit organ
regulation of cellular component size supramolecular fiber organ.

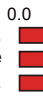

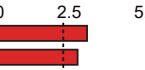

$-\log _{10}(P$-value $)$

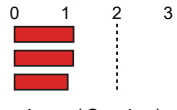

- $\log _{10}(Q$-value $)$

Cluster5 (Both-Down-regulated) DEG

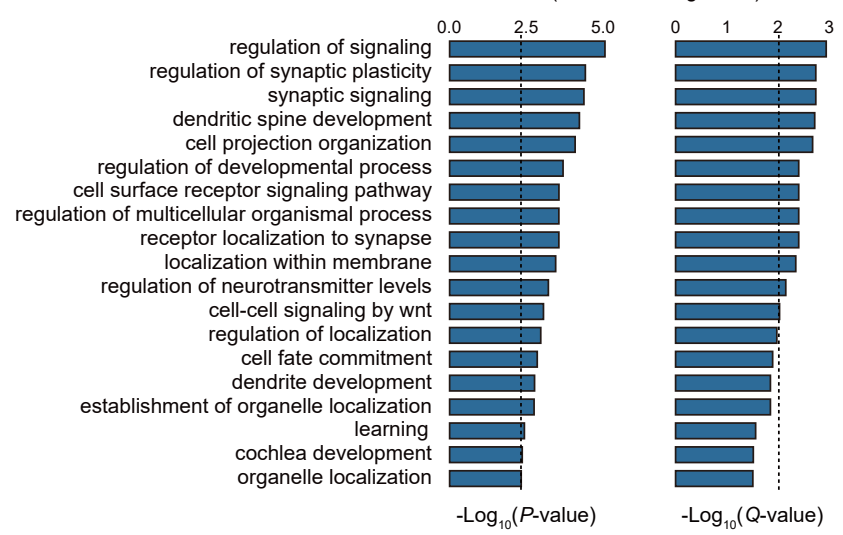

$-\log _{10}(P$-value $) \quad-\log _{10}(Q$-value $)$

B

Mouse embryonic fibroblasts NIH3T3
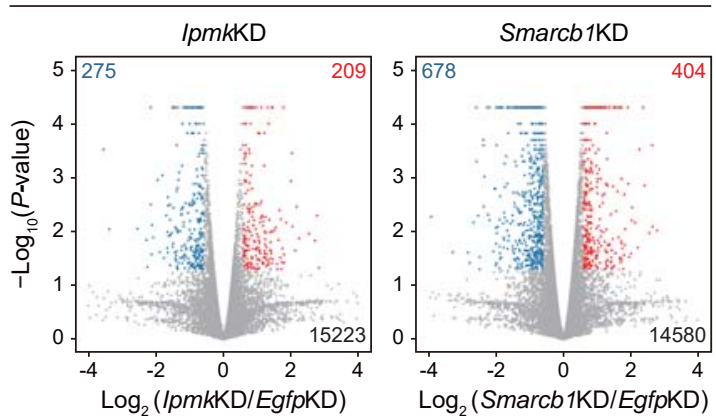

$\square$ Down-regulated $\square$ Not DEGs
C

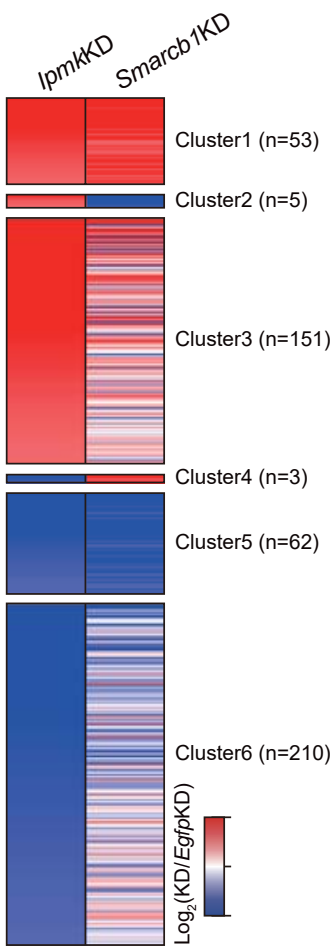

D

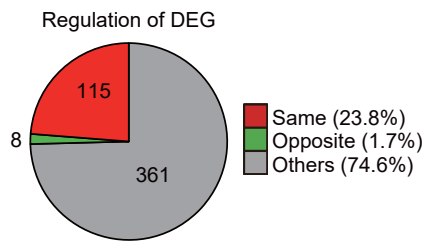

E

Up-regulated DEG IpmkKD Smarcb1KD

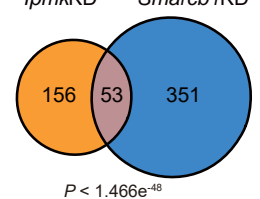

Down-regulated DEG IpmkKD Smarcb1KD

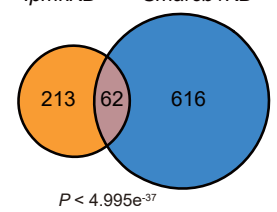




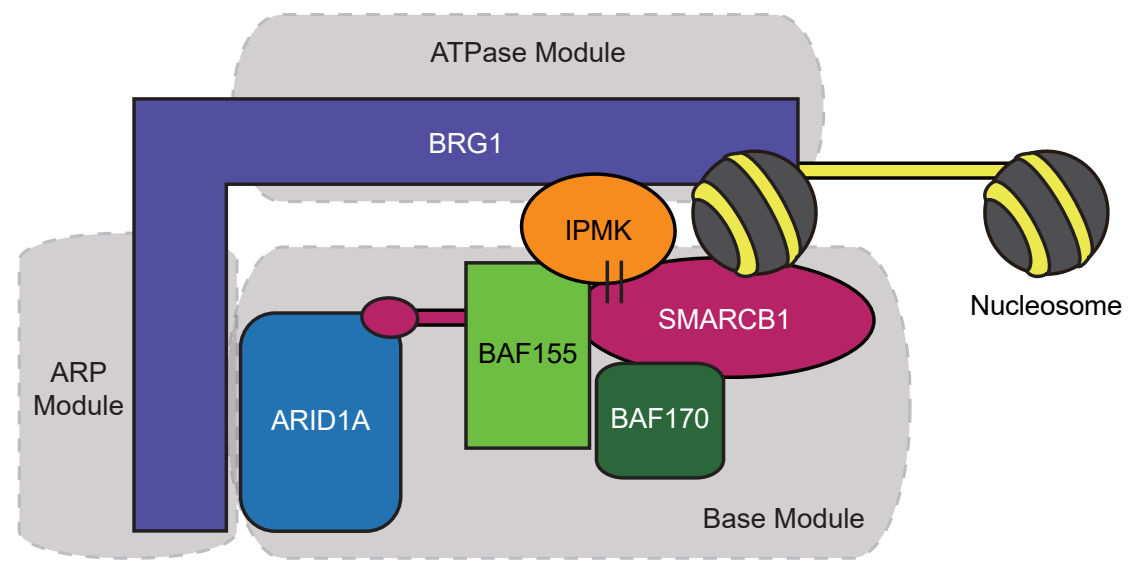


bioRxiv preprint doi: https://doi.org/10.1101/2021.09.15.460446; this version posted September 15, 2021. The copyright holder for this

preprint (which was not certified by peer review) is the author/funder, who has granted bioRxiv a license to display the preprint in perpetuity. It is made available under aCC-BY 4.0 International license.

Supplementary Table 1. Yeast two-hybrid screening assay using IPMK as bait

\begin{tabular}{|c|c|c|c|c|}
\hline \multirow{24}{*}{ Screening 1} & No. & Gene Symbol & Description & GenBank \\
\hline & 1 & $\mathrm{CLU}$ & Clusterin & NM_001831 \\
\hline & 2 & HSPD1 & Heat Shock 60kDa Protein 1 (Chaperonin) & NM_199440 \\
\hline & 3 & PSMD2 & Proteasome (Prosome, Macropain) 26S Subunit, non-ATPase, 2 & NM_002808 \\
\hline & 4 & PCDHGC4 & Protocadherin Gamma Subfamily C, 4 & NM_018928 \\
\hline & 5 & KHDRBS1 & KH Domain Containing, RNA Binding, Signal Transduction Associated 1 & NM_006559 \\
\hline & 6 & SMARCB1 & SWI/SNF Related, Matrix Associated, Actin Dependent Regulator of Chromatin, Subfamily b, Me & NM 001007468 \\
\hline & 7 & CTSD & Cathepsin D & NM_001909 \\
\hline & 8 & CYP46A1 & Cytochrome P450, Family 46, Subfamily A, Polypeptide 1 & NM 006668 \\
\hline & 9 & RNF208 & Ring Finger Protein 208 & NM_031297 \\
\hline & 10 & SPARCL1 & SPARC-like 1 (hevin) & NM_004684 \\
\hline & 11 & COL11A2 & Collagen, Type XI, Alpha 2 & NM_080681 \\
\hline & 12 & TNRC6B & Trinucleotide Repeat Containing 6B & NM_001162501 \\
\hline & 13 & DLG4 & Discs, Large Homolog 4 & NM_001128827 \\
\hline & 14 & RBM5 & RNA Binding Motif Protein 5 & NM_005778 \\
\hline & 15 & ATRX & Alpha Thalassemia/Mental Retardation Syndrome X-linked & NM 138270 \\
\hline & 16 & GAPDH & Glyceraldehyde-3-phosphate Dehydrogenase & NM_001256799 \\
\hline & 17 & B4GALNT1 & Beta-1,4-N-acetyl-galactosaminyl Transferase 1 & NM_001478 \\
\hline & 18 & DRG2 & Developmentally Regulated GTP Binding Protein 2 & NM_001388 \\
\hline & 19 & PLD3 & Phospholipase D Family, Member 3 & NM_012268 \\
\hline & 20 & GLUL & Glutamate-ammonia Ligase & NM_001033056 \\
\hline & 21 & APLP1 & Amyloid Beta (A4) Precursor-like Protein 1 & NM_005166 \\
\hline & 22 & SEPT4 & Septin 4 & NM 004574 \\
\hline & 23 & PSMF1 & Proteasome (Prosome, Macropain) Inhibitor Subunit 1 (PI31) & NM_178578 \\
\hline
\end{tabular}

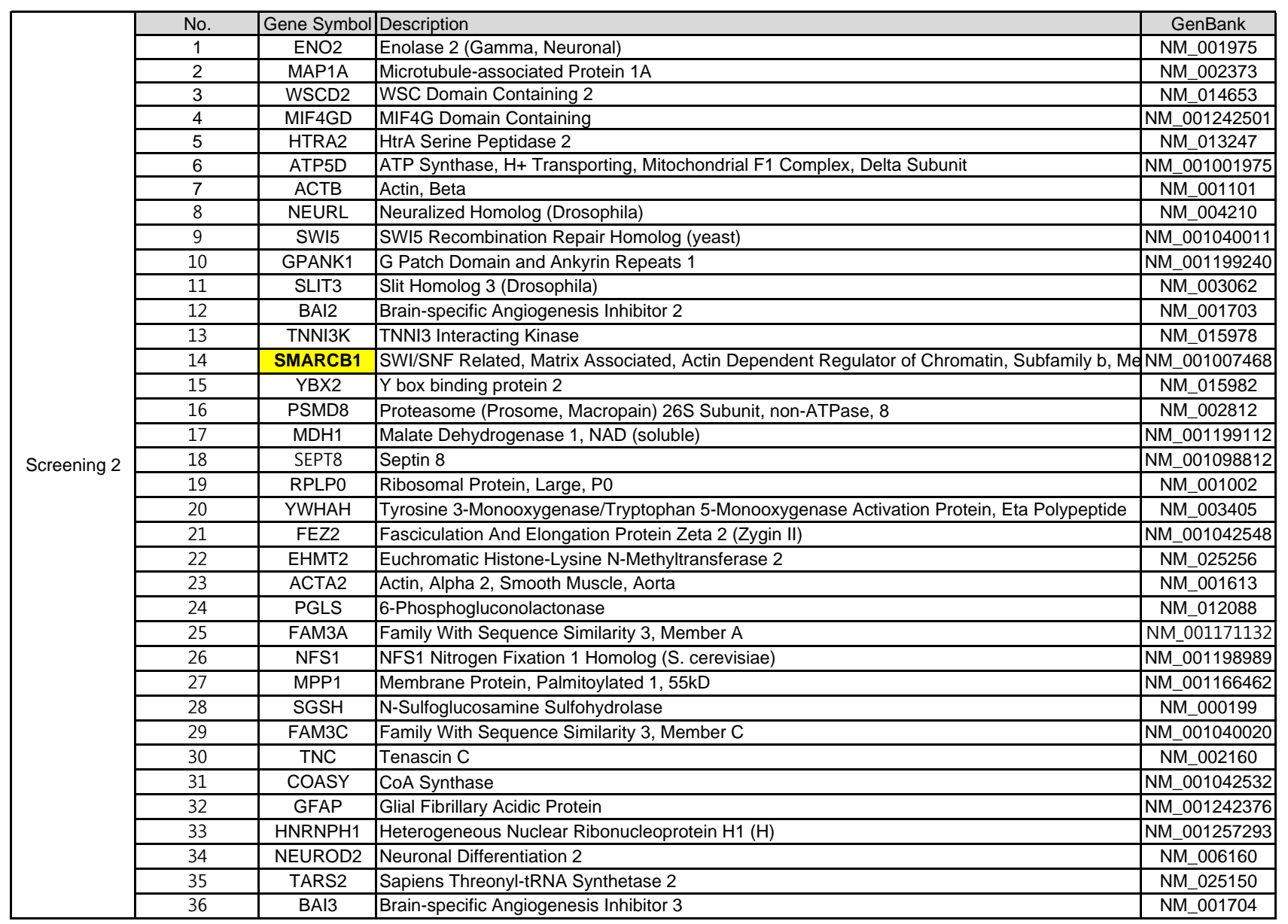


bioRxiv preprint doi: https://doi.org/10.1101/2021.09.15.460446; this version posted September 15, 2021. The copyright holder for this preprint (which was not certified by peer review) is the author/funder, who has granted bioRxiv a license to display the preprint in perpetuity. It is made available under aCC-BY 4.0 International license.

Supplementary Table 2. Enriched protein complex-based sets from APEX2-mediated proximity labeling

\begin{tabular}{|l|c|c|c|}
\hline \multicolumn{1}{|c|}{ Complex name } & Set size & Candiates contained & q-value \\
\hline BRG1-SIN3A-HDAC containing SWI/SNF remodeling complex I & 11 & $7(63.6 \%)$ & $4.83 \mathrm{E}-07$ \\
\hline BRG1-SIN3A complex & 14 & $7(50.0 \%)$ & $4.00 \mathrm{E}-06$ \\
\hline Brg1-associated complex II & 7 & $5(71.4 \%)$ & $1.60 \mathrm{E}-05$ \\
\hline BRG1-associated complex & 9 & $5(55.6 \%)$ & $6.98 \mathrm{E}-05$ \\
\hline BAF complex & 9 & $5(55.6 \%)$ & $6.98 \mathrm{E}-05$ \\
\hline PBAF complex (Polybromo- and BAF containing complex) & 9 & $5(55.6 \%)$ & $6.98 \mathrm{E}-05$ \\
\hline BRM-SIN3A complex & 15 & $6(40.0 \%)$ & $8.10 \mathrm{E}-05$ \\
\hline EBAFa complex & 10 & $5(50.0 \%)$ & 0.000104312 \\
\hline BAF complex & 13 & $5(38.5 \%)$ & 0.000329586 \\
\hline Brg1-based SWI/SNF complex & 4 & $3(75.0 \%)$ & 0.000720336 \\
\hline EBAFb complex & 10 & $4(40.0 \%)$ & 0.001110216 \\
\hline BRM-associated complex & 10 & $4(40.0 \%)$ & 0.001110216 \\
\hline
\end{tabular}


bioRxiv preprint doi: https://doi.org/10.1101/2021.09.15.460446; this version posted September 15, 2021. The copyright holder for this preprint (which was not certified by peer review) is the author/funder, who has granted bioRxiv a license to display the preprint in perpetuity. It is made available under aCC-BY 4.0 International license.

Supplementary Table 3. Clustering analysis of DEG in mouse embryonic stem cells

\begin{tabular}{|c|c|c|c|}
\hline Ipmk KD & Smarcb1 KD & Number of genes & Percentage \\
\hline \multirow{3}{*}{ Up-regulated } & Up-regulated & 50 & $23.0 \%$ \\
\cline { 2 - 4 } & Down-regulated & 1 & $0.5 \%$ \\
\cline { 2 - 4 } & Not DEGs & 166 & $76.5 \%$ \\
\hline \multirow{3}{*}{ Down-regulated } & Up-regulated & 2 & $0.7 \%$ \\
\cline { 2 - 4 } & Down-regulated & 47 & $15.7 \%$ \\
\cline { 2 - 4 } & Not DEGs & 251 & $83.7 \%$ \\
\hline
\end{tabular}


bioRxiv preprint doi: https://doi.org/10.1101/2021.09.15.460446; this version posted September 15, 2021. The copyright holder for this preprint (which was not certified by peer review) is the author/funder, who has granted bioRxiv a license to display the preprint in perpetuity. It is made available under aCC-BY 4.0 International license.

Supplementary Table 4. Clustering analysis of DEG in mouse fibroblasts

\begin{tabular}{|c|c|c|c|}
\hline Ipmk KD & Smarcb1 KD & Number of genes & Percentage \\
\hline \multirow{3}{*}{ Up-regulated } & Up-regulated & 53 & $24.4 \%$ \\
\cline { 2 - 4 } & Down-regulated & 5 & $2.3 \%$ \\
\cline { 2 - 4 } & Not DEGs & 151 & $69.6 \%$ \\
\hline \multirow{3}{*}{ Down-regulated } & Up-regulated & 3 & $1.0 \%$ \\
\cline { 2 - 4 } & Down-regulated & 62 & $20.7 \%$ \\
\cline { 2 - 4 } & Not DEGs & 210 & $70.0 \%$ \\
\hline
\end{tabular}

